Aus der Abteilung Allgemeinmedizin

(Prof. Dr. med. M. M. Kochen, MPH, FRCGP)

im Zentrum Innere Medizin

der Medizinischen Fakultät der Universität Göttingen

\title{
Warum informieren sich Kopfschmerzpatienten im Internet?
}

Inhaltsanalyse und Teilnehmerbefragung eines webbasierten Forums

\author{
INAUGURAL - DISSERTATION \\ zur Erlangung des Doktorgrades \\ der Medizinischen Fakultät \\ der Georg-August-Universität zu Göttingen
}

vorgelegt von

Sophie Zitterbart

aus

Göttingen

Göttingen 2009 
D e k a n:

Prof. Dr. med. C. Frömmel

I. Berichterstatter: Prof. Dr. med. M. Scherer

II. Berichterstatterl in:

III. Berichterstatterl in:

Tag der mündlichen Prüfung: 


\section{Inhalt}

Inhalt III

Abbildungsverzeichnis VI

Tabellenverzeichnis $\mathrm{VI}$

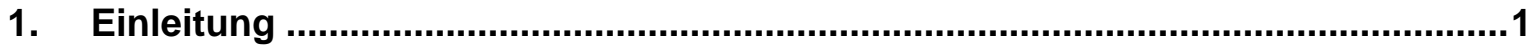

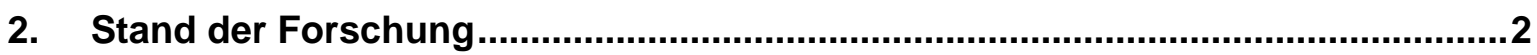

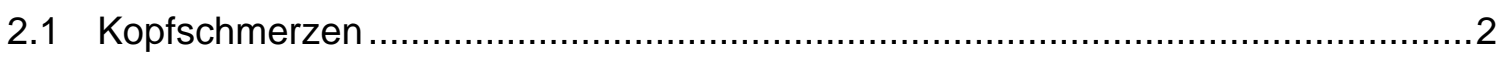

2.1.1 Definition und Klassifikation ........................................................... 2

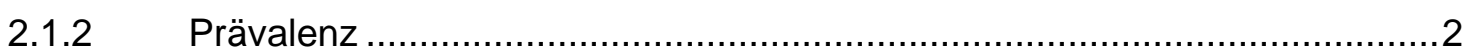

2.1.3 Sozioökonomische Bedeutung von Kopfschmerzen ...............................

2.2 Versorgungssituation von Kopfschmerzen in der Hausarztpraxis ....................... 4

2.3 Das Internet als Beratungs- und Informationsmedium.................................... 6

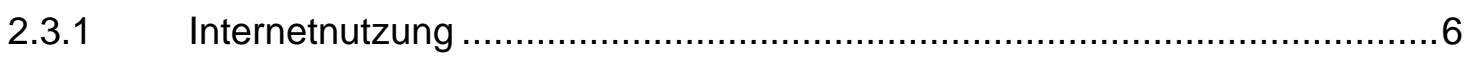

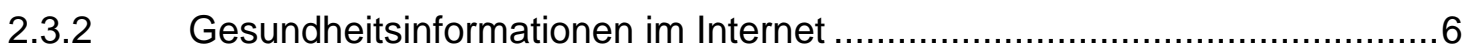

2.3.3 Reflexion der unbeschränkten Informationsgewinnung in der Literatur .......7

2.4 Die Rolle des Internets in der medizinischen Betreuung von Patienten ................9

2.4.1 Internetnutzung im medizinischen Praxisalltag ..................................... 9

2.4.2 Gründe von Patienten zur Internetnutzung für medizinische Zwecke........10

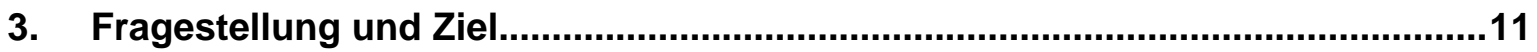

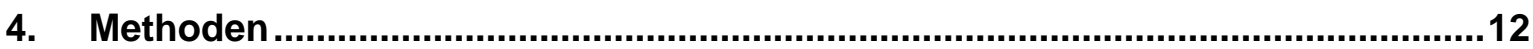

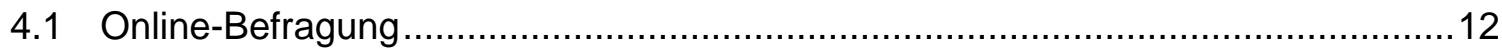

4.1.1 Methodische Anlage der Untersuchung ............................................

4.1.2 Genehmigung durch die Ethik-Kommission ....................................... 12

4.1.3 Teilnehmende Nutzer ............................................................. 13

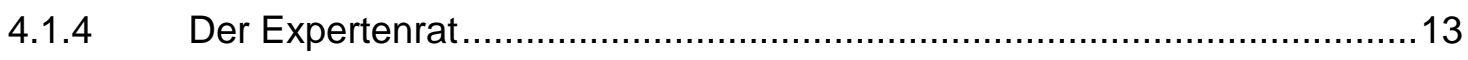

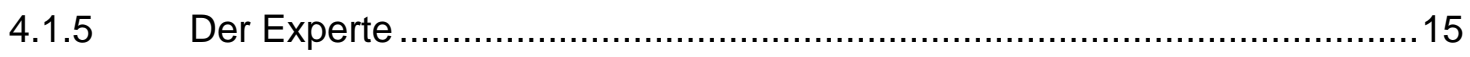

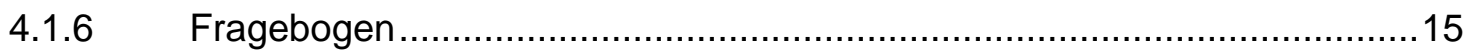

4.1.7 Durchführung der Untersuchung .........................................................20

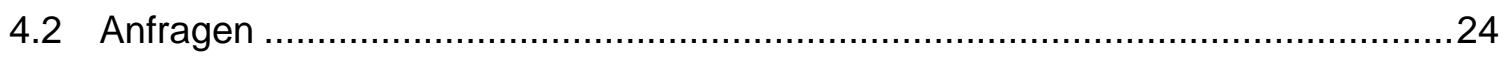

4.2.1 Kategorien der Anfragenanalyse .................................................... 24

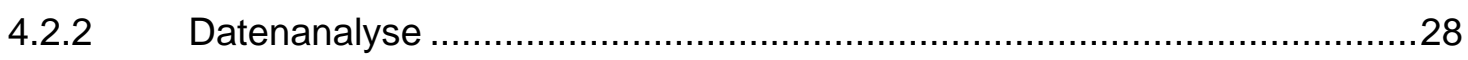




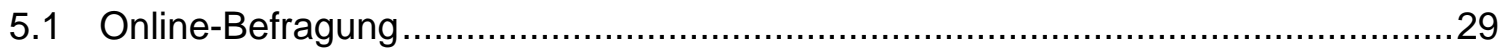

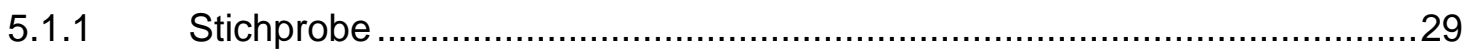

5.1.2 Stichprobenpopulation der Online-Befragung (soziodemogr. Daten) ........29

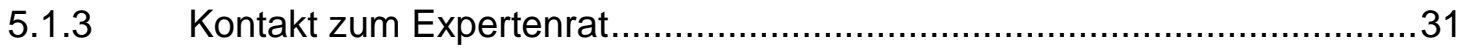

5.1.4 Internetsprechstunden, Internetverhalten .......................................... 31

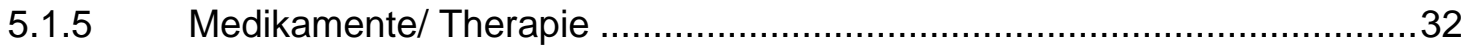

5.1.6 Krankengeschichte der Studienteilnehmer ....................................... 33

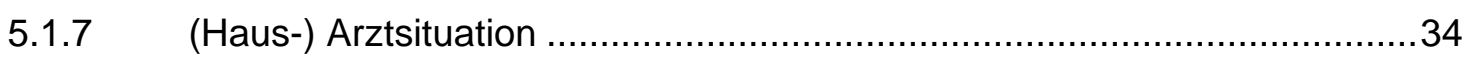

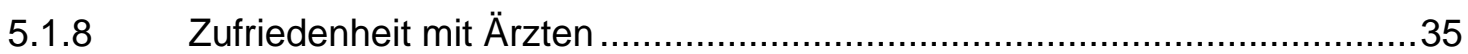

5.1.9 Fühlen sich die Betroffenen mit ihren Beschwerden ernst genommen? ....36

5.1.10 Gründe für die Nutzung des Expertenrats im Internet.............................37

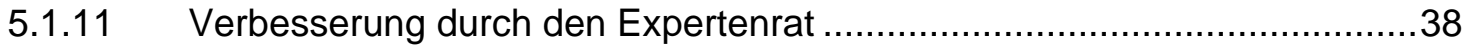

5.1.12 Fragebogen zur Differenzierung der Kopfschmerzen ..............................39

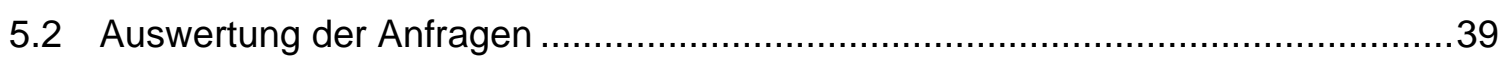

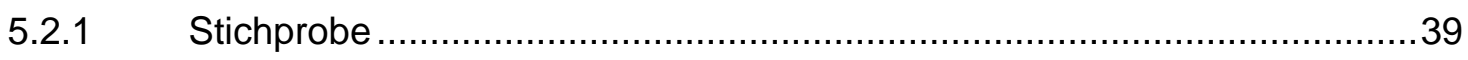

5.2.2 Stichprobenpopulation (soziodemographische Daten) ...........................40

5.2.3 Charakteristika der Anfragen ...................................................................40

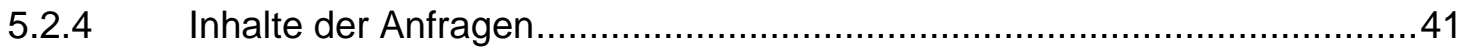

5.2.5 Kritik und Kommentare ......................................................................

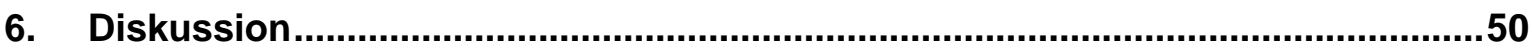

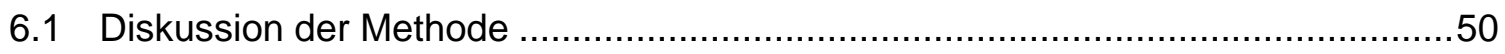

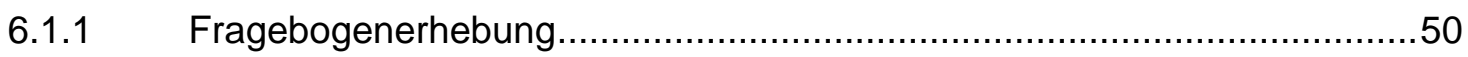

6.1.2 Inhaltsanalytische Auswertung der Anfragen .........................................51

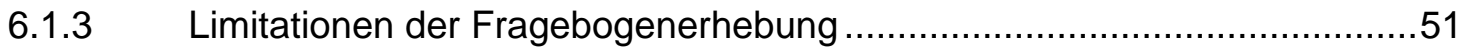

6.1.4 Limitationen der inhaltsanalytischen Auswertung .................................54

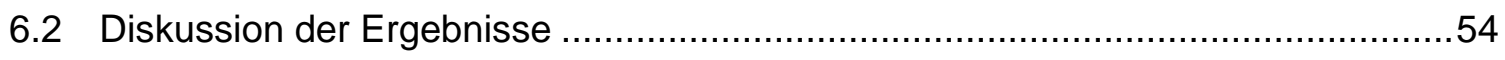

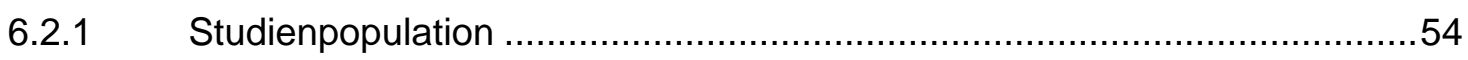

6.2.2 Leitliniengerechte Therapie der Nutzer ................................................56

6.2.3 Der chronische (Kopf-) Schmerzpatient - ein schwieriger Patient? ...........56

6.2.4 Patientenzufriedenheit mit der ärztlichen Versorgung .............................57

6.2.5 Patientenwünsche an ihre medizinische Versorgung ............................58

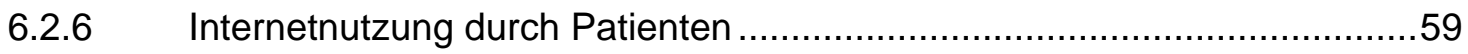

6.2.7 Verbesserung der medizinischen Versorgung durch das Internet ............60 


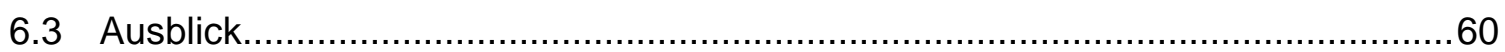

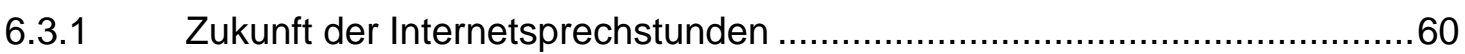

6.3.2 Mögliche Verbesserung der hausärztlichen Versorgung ..........................62

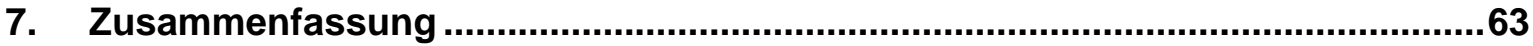

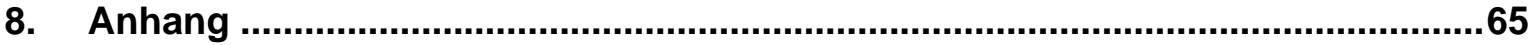

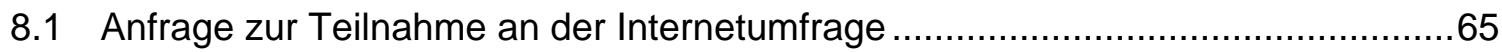

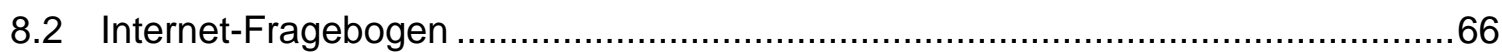

8.3 Grundauszählung des Internet-Fragebogens ….............................................. 73

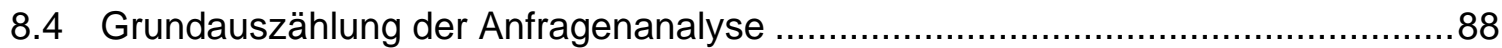

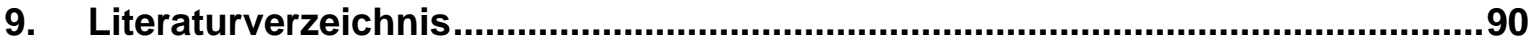




\section{Abbildungsverzeichnis}

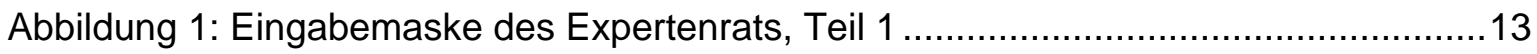

Abbildung 2: Eingabemaske des Expertenrats, Teil 2 ................................................... 14

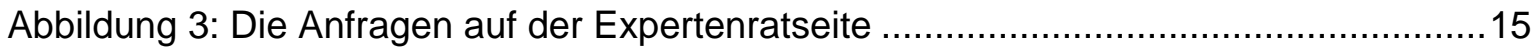

Abbildung 4: Online-Präsentation des Internet-Fragebogens...................................... 16

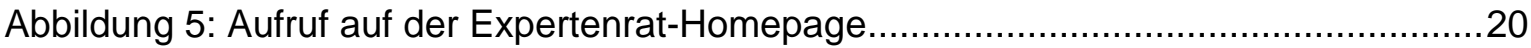

Abbildung 6: Homepage des Expertenrats „Migräne und Kopfschmerz" mit Teaserbox...22

Abbildung 7: Wie sehr fühlen sich die Betroffenen ernst genommen durch...................37

Abbildung 8: Gründe für die Nutzung des Expertenrats im Internet ................................38

Abbildung 9: Sicherheitshinweis über abgelaufenes Sicherheitszertifikat ........................53

\section{Tabellenverzeichnis}

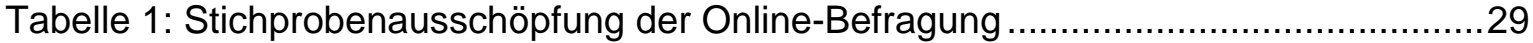

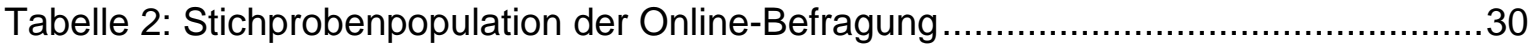

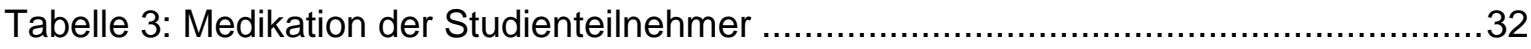

Tabelle 4: Zusätzliche Erkrankungen der Studienteilnehmer ....................................... 34

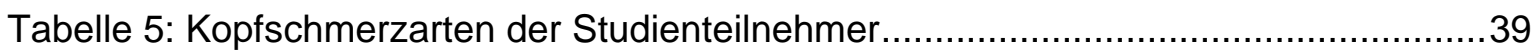

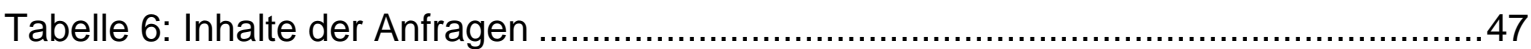




\section{Einleitung}

Kopfschmerzen sind ein häufiges Symptom in der Bevölkerung, werden aber verhältnismäßig selten als Beratungsanlass in Hausarztpraxen thematisiert. Möglicherweise empfinden Patienten diese Beschwerden als Bagatelle, mit der man den Hausarzt nicht „belästigt"; eventuell sind Patienten aber auch unzufrieden mit der bisherigen Beratung ihres Hausarztes bei Kopfschmerzen.

Kopfschmerzen können in den unterschiedlichsten Formen und Zusammenhängen auftreten. Grundsätzlich muss man zwischen primärem und sekundärem Kopfschmerz unterscheiden, wobei der sekundäre Kopfschmerz als Symptom einer Grunderkrankung auftritt. Bei dem primären Kopfschmerz handelt es sich meist um Migräne oder den episodischen Spannungskopfschmerz. Die Prävalenz nimmt vor allem bei Kindern stetig zu, so dass die adäquate und ausreichende Behandlung von Kopfschmerzen ein großes Problem der heutigen Gesundheitspolitik darstellt.

Die hausärztliche Betreuung von Kopfschmerzpatienten ist aufwendig und erfordert neben viel Engagement auch einen großen Zeitaufwand vom jeweiligen Allgemeinarzt. Oft weist die Behandlung daher Mängel vor allem hinsichtlich der psychosozialen Versorgung auf. Die technische Entwicklung der letzten Jahrzehnte hat die Möglichkeiten der Informationsgewinnung über medizinische Themen revolutioniert (Peterlin et al. 2007). Die betroffenen Patienten suchen immer mehr nach zusätzlicher Hilfe in den Medien. Eine zunehmend wichtige Rolle hat hierbei vor allem das Internet (Peterlin et al. 2008), das immer mehr als Medium zur medizinischen Beratung (nicht nur) bei Kopfschmerzen genutzt wird. Hier finden die Betroffenen viele Adressen und Foren, in denen ein reger Austausch von Erfahrungen und Informationen stattfindet. Neben der gegenseitigen Hilfe untereinander finden die Patienten hier auch kompetente Beratung durch Fachärzte, die auf persönliche Fragen individuell eingehen.

Das in dieser Arbeit untersuchte Internetforum (Expertenrat "Migräne und Kopfschmerz") ist auf der Lifeline-Homepage der Business Solutions Medicine Online (BSMO), einer Tochtergesellschaft der Fachverlagsgruppe Springer Science+Business Media und der DGN Services $\mathrm{GmbH}$, zu finden und hat sich zur Aufgabe gemacht, Fragen von InternetUsern zum Thema „Kopfschmerz“ zu beantworten. Als Experte fungiert hierbei ein Facharzt für Allgemeinmedizin, Neurologie und Kinder- und Jugendpsychiatrie, der die gestellten Fragen persönlich beantwortet. 


\section{Stand der Forschung}

\subsection{Kopfschmerzen}

\subsubsection{Definition und Klassifikation}

Nach der International Headache Society werden Kopfschmerzen zunächst in primäre und sekundäre Kopfschmerzformen unterteilt (Headache Classification Subcommittee of the International Headache Society 2004). Bei den primären Formen wird weiter unterschieden zwischen Migräne, Kopfschmerzen vom Spannungstyp, Clusterkopfschmerz und anderen trigemino-autonomen Kopfschmerzformen sowie anderen Formen primärer Kopfschmerzen (z. B. Hustenkopfschmerz oder Kopfschmerzen nach körperlicher Anstrengung). Die sekundären Kopfschmerzformen umfassen die Arten, die auf eine andere Grunderkrankung zurückzuführen sind, z. B. ein stattgehabtes Trauma, Entzündungen, Tumore etc. Neben primären und sekundären Kopfschmerzen werden die kranialen Neuralgien und zentrale Ursachen von Gesichtsschmerzen gesondert betrachtet. Hierzu zählt zum Beispiel die Trigeminusneuralgie. Alle anderen ansonsten nicht zu klassifizierenden Kopfschmerzen werden in der Gruppe der anderen Kopfschmerzen, kranialen Neuralgien und zentralen oder primären Gesichtsschmerzen zusammengefasst. Hierunter fallen vor allem jene Kopfschmerzen, die erstmalig beschrieben werden oder über die nicht ausreichend Informationen verfügbar sind.

\subsubsection{Prävalenz}

Die Prävalenz von Kopfschmerzen variiert vor allem in Hinblick auf das Alter, das Geschlecht und die Herkunft der Patienten. Die Lebenszeitprävalenz von Kopfschmerzen beträgt $93 \%$ bei Männern und $99 \%$ bei Frauen (Rasmussen et al. 1991). Migräne (ungeachtet der Form und Häufigkeit) tritt bei 8 - $12 \%$ der Männer und ca. 25 \% der Frauen zumindest einmal im Leben auf. Der Kopfschmerz vom Spannungstyp ist sehr viel häufiger vertreten. 69 \% der Männer und 88 \% der Frauen sind mindestens einmal in ihrem Leben hiervon betroffen (Rasmussen et al. 1991). 6 - $8 \%$ der Männer und 15 - $18 \%$ der Frauen gaben an, mindestens einmal während des Vorjahres an Migräne gelitten zu haben (Rasmussen et al. 1991, Stewart et al. 1994, Lipton et al. 2001, Steiner et al. 2003). Frauen erkranken somit zwei bis drei Mal so häufig an Migräne als Männer (Rasmussen et al. 1991). Es werden in der Literatur jedoch auch Zahlen angegeben, nach denen Frauen bis zu sechs Mal häufiger betroffen sind (Lyngberg et al. 2005 a). Die häufigsten Auslöser einer Migräne sind einer dänischen Studie zufolge Stress und mentale Anspannung, zu den häufigsten Symptomen einer Migräneaura gehören Sehstörungen (Rasmussen und Olesen 1992). 
Ein besonderer Aspekt im Auftreten von Kopfschmerzen ist, dass auch die Herkunft bzw. die Rasse eine Rolle spielt. Menschen kaukasischer Herkunft erkranken häufiger als Angehörige anderer Rassen (Steiner et al. 2003). Es lassen sich jedoch nicht nur kontinentale, sondern auch innerhalb eines Landes regionale Unterschiede feststellen, wie 2008 in einer Studie der Deutschen Migräne- und Kopfschmerzgesellschaft herausgefunden wurde (Pfaffenrath et al. 2008). Demgegenüber steht eine etwas ältere deutsche Studie, die zeigte, dass in Deutschland in den einzelnen Bundesländern kein Unterschied hinsichtlich der Prävalenz der Migräne besteht, wohl aber beim Vergleich der Prävalenzen in Städten unterschiedlicher Größe (Göbel et al. 1994). In Städten mit 20.000 bis 50.000 Einwohnern trat Migräne bei $31 \%$ der Bewohner auf, in Städten mit Einwohnerzahlen von 100.000 bis 500.000 nur bei $24 \%$. Bei Spannungskopfschmerz sowohl des chronischen als auch des episodischen Typs zeigten sich keinerlei ortsspezifische Unterschiede.

Die Prävalenz der Migräne korreliert zudem auch mit dem Alter der Patienten, vor allem Personen mittleren Alters sind betroffen. Es findet sich ein Altersgipfel bei ca. $30-49$ Lebensjahren (Molarius und Tegelberg 2006, Steiner et al. 2003, Lipton et al. 2002). Ältere Menschen sind seltener betroffen, weisen dann jedoch häufiger atypische, vegetative Symptome wie Mundtrockenheit und Blässe auf, so dass die Diagnosestellung häufig erschwert ist (Martins et. al 2006). 10 - $25 \%$ der Kinder und heranwachsenden Jugendlichen leiden an Kopfschmerzen (Antilla 2006), wobei Jungen beim Erstauftreten einer Migräne jünger sind als Mädchen (Breslau und Rasmussen 2001).

Betrachtet man die Entwicklung der Prävalenz über mehrere Jahre (wie in einer dänischen Studie geschehen), so fällt auf, dass die Prävalenz von Kopfschmerzen des Spannungstyps im Gegensatz zur Prävalenz der Migräne deutlich gestiegen ist (79- $87 \%)$ (Lyngberg et al. 2005 b). Bei der Migräne fält hingegen auf, dass die Anzahl der Patienten, die 14 Tage oder mehr im Jahr an einem Migräneanfall leiden, angestiegen ist (12 38 \%). Die Prävalenz der Migräne blieb jedoch in den letzten Jahren konstant (Lipton et al. 2002, Lipton et al. 2007, Freitag 2007).

\subsubsection{Sozioökonomische Bedeutung von Kopfschmerzen}

Kopfschmerzen (und im Besonderen Migräne) stellen nicht nur für den einzelnen Betroffenen eine große Belastung dar, sie spielen durchaus auch eine bedeutende sozioökonomische Rolle. Migräne ist einer der 20 wichtigsten Gründe für Behinderungen und körperliche Einschränkungen weltweit und ist verantwortlich für einen großen Teil der nichtschwerwiegenden krankheitsbezogenen Belastungen (Leonardi et al. 2005). Bei Frauen gehört sie gar zu den fünf häufigsten Gründen für körperliche Einschränkungen (Stovner 
et al. 2007). So müssen neben den direkten, durch Kopfschmerz entstehenden Kosten der medizinischen Versorgung auch die indirekten, durch Arbeitsunfähigkeiten, eingeschränkte Produktivität und Effektivität der Betroffenen verursachten Kosten bedacht werden (Rasmussen 1999). Durchschnittlich entfallen pro betroffenem Patienten 7,6 Arbeitsstunden in drei Monaten durch einen Migräneanfall (Kobak et al. 2005). 58,1 \% der Patienten mit chronischer Migräne und 18,2 \% der Patienten mit episodischer Migräne berichten über Einschränkungen im Haushalt für mindestens fünf Tage innerhalb von drei Monaten (Bigal et al. 2008). Schätzungen in Amerika gehen davon aus, dass jährlich \$ $13-17$ Milliarden Kosten durch Migräne entstehen (Goldberg 2005). Pro Migränepatient werden so $\$ 2571$ pro Jahr mehr ausgegeben als für Nicht-Betroffene (Hawkins et al. 2008). Die Kosten umfassen vor allem die ambulante Patientenversorgung und Arzneimittelverschreibungen. Während des letzten Jahrzehnts sind die hohen Kosten für die Versorgung von Kopfschmerzpatienten jedoch nicht weiter angestiegen (Pradalier et al. 2004).

\subsection{Versorgungssituation von Kopfschmerzen in der Hausarztpraxis}

Wichtigste medizinische Ansprechpartner für die Kopfschmerzpatienten sind Hausärzte. Sie werden in der Regel als erstes aufgesucht, wenn Patienten aufgrund ihrer Kopfschmerzen medizinische Betreuung wünschen (Sadovsky und Dodick 2005). Außerdem sind Kopfschmerzen einer der häufigsten Gründe von Patienten, einen Arzt aufzusuchen (Neu 2001). Die Patienten möchten neben Therapievorschlägen vor allem die Ursache ihrer Kopfschmerzen erfahren und suchen die Bestätigung, dass der Kopfschmerz kein Ausdruck einer malignen Grunderkrankung ist (MacGregor 1997). 68 \% der von Migräne betroffenen Frauen und 57 \% der betroffenen Männer gaben an, zumindest einmal aufgrund ihrer Kopfschmerzen einen Arzt aufgesucht zu haben (Lipton et al. 1998). Ungefähr $72 \%$ der Migränepatienten wenden sich hierbei zunächst an ihren behandelnden Hausarzt (Gibbs et al. 2003, Walling et al. 2005). Hierbei fällt auf, dass Frauen den größten Anteil ausmachen, nämlich fast $80 \%$. Besonders jüngere Frauen (15. - 24. Lebensjahr) suchen häufiger als andere bei Kopfschmerzen einen Arzt auf (Latinovic et al. 2006). Die meisten Patienten bekommen von ihrem behandelnden Arzt rezeptfreie Medikamente verordnet (Lipton et al. 2003 b).

Dennoch wird die Mehrzahl der Kopfschmerzerkrankungen außerhalb des ärztlichen Settings behandelt, da nur ein geringer Anteil der Betroffenen ärztliche Hilfe in Anspruch nimmt. Nur schätzungsweise ein Drittel der Betroffenen wendet sich aufgrund der Kopfschmerzen an einen Arzt (Walling et al. 2005), die Mehrzahl sucht keinen medizinischen Rat (Thomas et al. 2004). Eine neuere Studie aus Deutschland bestätigt diese Angaben, 
hier hatten nur $42 \%$ der befragten Migränepatienten bisher einen Arzt konsultiert (Radtke und Neuhauser 2009). Dabei berichten 61 \% derjenigen, die aufgrund ihrer Kopfschmerzen noch nie einen Arzt aufgesucht haben, über schwere und sehr schwere Schmerzen und $67 \%$ geben gar an, aufgrund ihrer ausgeprägten Kopfschmerz-Symptomatik das Bett hüten zu müssen (Lipton et al. 1998). Jedoch können Migränepatienten nach spezifischer Beratung ihre Erkrankung häufig selbst diagnostizieren (Göbel et al. 2006).

Ein weiteres Problem ist die ausgeprägte Non-Compliance der Kopfschmerz-Patienten. $40 \%$ der Patienten erscheinen kein zweites Mal in der Sprechstunde, so dass die Beobachtung des weiteren Verlaufs nicht möglich ist. Zudem wird eine große Zahl der ausgestellten Rezepte nicht eingelöst, nämlich $20 \%$ der verschriebenen Triptane und 25 - 50 $\%$ der Medikamente zur Migräneprophylaxe (Baskin 2007).

Die Anzahl der ärztlichen Konsultationen im Zusammenhang mit dem Hauptsymptom „Kopfschmerz“ verdoppelte sich im Zeitraum von 1990 bis 1998, nämlich von 9,4 Besuchen pro 1000 Personen auf 18 Besuche pro 1000 Personen. Die durchschnittliche Anzahl an Arztkonsultationen der einzelnen Patienten hängt stark von der jeweiligen Kopfschmerzform ab, wobei Migränepatienten die Praxis am häufigsten aufsuchen (Hasse et al. 2002). Trotz neuerer Behandlungsmöglichkeiten wird die Vielzahl der Patienten meist mit älteren und wahrscheinlich weniger effektiven Medikamenten behandelt (Gibbs et al. 2003). Auch scheinen Medikamente zur Migräneprophylaxe, wie z. B. Betablocker, noch zu selten eingesetzt zu werden. Die volle Bandbreite der möglichen Medikamente wird nicht ausgenutzt und häufig werden unzureichende Maßnahmen zur Prophylaxe ergriffen (Stark et al. 2007). Daher scheinen evidenzbasierte Trainingsprogramme und Fortbildungen sowie Leitlinien zur Behandlung und Vorbeugung von Kopfschmerzen sinnvoll (Bianco et al. 2005). In Deutschland existieren von der Kommission „Leitlinien der Deutschen Gesellschaft für Neurologie" erstellte Leitlinien hinsichtlich der Diagnostik von Kopfschmerzen und der Therapie der Migräneattacken sowie der Migräneprophylaxe (Kommission „Leitlinien der Deutschen Gesellschaft für Neurologie“ 2008 a, b). Eine S3Leitlinie der Deutschen Gesellschaft für Allgemein- und Familienmedizin (DEGAM) ist derzeit in Arbeit (Mildenstein 2009). 


\subsection{Das Internet als Beratungs- und Informationsmedium}

\subsubsection{Internetnutzung}

Das Internet hat in den letzten Jahren erheblich an Bedeutung zugenommen und Einzug in das alltägliche Leben gefunden. Laut Statistischem Bundesamt besaßen im Jahr 2007 $72,2 \%$ der deutschen Haushalte mindestens einen Computer und 64,9\% der Haushalte besaßen einen eigenen Internetzugang, wobei hiervon 66,2 \% die schnellere Internetverbindung DSL nutzten (Statistisches Bundesamt Deutschland 2008 a, b). Hochgerechnet nutzten 200750.635 .000 deutsche Bürger das Internet (61,6 \% der deutschen Gesamtbevölkerung). $61,4 \%$ gaben an, das Internet täglich oder zumindest fast täglich zu nutzen, zumeist zu Hause (89,3 \%). Hauptsächlich wurde das Internet für das private Senden und Empfangen von E-Mails (86,0 \%) und zur Informationssuche über Waren und Dienstleistungen genutzt (83,6 \%). Ein Großteil benutzte das Internet außerdem, um Informationen zu Gesundheitsthemen zu suchen (52,6 \%).

Eine genaue Zahl der weltweit existierenden Internetseiten lässt sich aufgrund des stetigen Wachstums des Informationstechnologie-Sektors schwer festlegen. Die Firma Netcraft (www.netcraft.com), eine Internet Services Company aus Bath, England, spricht in inrem Web Server Survey vom September 2008 von weltweit 181.277.835 verschiedenen Servern, die alle Internetseiten bereitstellen (Netcraft 2008). Die Internet-Suchmaschine Google gibt in ihrem Unternehmensprofil ca. 8 Milliarden durchsuchte Internetseiten an (Google Unternehmensprofil 2008, zugegriffen am 28.10.2008).

\subsubsection{Gesundheitsinformationen im Internet}

Allein im deutschsprachigen Raum gibt es ein vielfältiges Angebot an Internetseiten unterschiedlicher Art, die sich mit Gesundheitsthemen befassen. Zum einen gibt es kommerzielle Seiten, die in Form von Gesundheitsportalen Informationen zu den unterschiedlichsten medizinischen Themen verbreiten (z. B. www.netdoctor.de, www.medizinforum.de oder www.gesundheit.de). Auf diesen Seiten finden sich häufig so genannte Gesundheitslexika, in denen Ratsuchende gezielt zu verschiedenen Themen Informationen suchen können. Auch Expertenforen oder Chats, in denen individuell Fragen gestellt werden können und oftmals ein reger Austausch von Betroffenen erfolgt, sind dieser Art von Seiten häufig angegliedert. Außerdem gibt es eine Vielzahl an Internetauftritten, die von Krankenhäusern, Arztpraxen, medizinischen Universitäten, Krankenkassen und anderen medizinbezogenen Organisationen und Gesellschaften unterhalten werden. Hier werden oftmals gezielt Informationen präsentiert, die bestimmte Patientengruppen ansprechen sollen. Zudem existieren kostenpflichtige Internetangebote, auf denen gegen Ge- 
bühr Ärzte virtuell konsultiert werden können und unter anderem auch rezeptpflichtige Medikamente verschrieben werden (Bloom und lannacone 2006).

Die Informationsflut zum speziellen Thema „Kopfschmerz“ ist gewaltig, unter dem Suchbegriff „Kopfschmerz" werden 532.000 deutschsprachige Schlagworttreffer im Internet angezeigt (Google, zugegriffen am 01.02.2009). Die Schlagworte „Migräne“ und „Spannungskopfschmerz" ergeben 1.220.000 bzw. 48.600 Treffer. Der Begriff „Clusterkopfschmerz" findet sich 35.200 Mal auf deutschsprachigen Internetseiten (Google, jeweils zugegriffen am 01.02.2009).

\subsubsection{Reflexion der unbeschränkten Informationsgewinnung in der Literatur}

Die vielfältigen Möglichkeiten der Informationsgewinnung bergen neben den Vorteilen der unbegrenzten Recherche jedoch auch Risiken und Gefahren hinsichtlich möglicher Fehlinformationen oder Missinterpretationen. So stellt das Internet neben seinen unbegrenzten Möglichkeiten auch eine Gefahr hinsichtlich nicht-validierter Informationen dar (Genzen 1998). Zu dieser Überzeugung gelangt auch eine australische Studie, die zehn Vergleichsstudien zur medizinbezogenen Internetnutzung bezüglich deren wissenschaftlicher Qualität untersuchte (Besell et al. 2002). Dabei sei das Problem, dass Patienten nun die gleichen Informationsquellen nutzen können wie medizinisch ausgebildetes Personal, ohne diese Information allerdings hinsichtlich ihres Wahrheitsgehaltes objektiv prüfen zu können. Zudem seien die Effekte der Internetnutzung von Patienten in Bezug auf deren Wissensstand, Kenntnis über ihre Krankheit und ihr Verhalten nur unzulänglich untersucht. Zusätzliche Studien seien notwendig, um neben den Gründen der im Internet ratsuchenden Patienten vor allem auch die Vor- und Nachteile dieser Entwicklung zu untersuchen. Auf die Gefahr der Potenzierung von Ängsten durch unvalide und unreliable Informationen im Internet machen Smith et al. aufmerksam (2006). Der somit „verwundbare“, nach medizinischen Informationen suchende Internetnutzer sei angreifbar durch kommerzielle Seiten, z. B. von pharmazeutischen Unternehmen, und könne so finanziell belastet werden.

Webseiten enthalten häufig widersprüchliche Angaben zu der Art des dargebotenen Inhalts und es ist selten ersichtlich, welche Qualifikation die jeweiligen Betreiber der Seite besitzen (Recupero und Rainey 2006). Giffin untersuchte verschiedene Seiten aus dem angloamerikanischen Sprachraum hinsichtlich Aufbau und Themenvielfalt, allerdings wurde hier keine Stellung zu den Inhalten und deren Relevanz bzw. deren Richtigkeit genommen (2002). Anders Peroutka (2001), bei dessen Studie im Januar 2000 das Stichwort „headache“ in zehn verschiedene führende Internetsuchmaschinen eingegeben wur- 
de und die jeweils zehn erstgelisteten Seiten auf ihre Relevanz als Informationsquelle für Kopfschmerzpatienten hin untersucht wurden. Kernaussage der Studie ist, dass es Aufgabe der behandelnden Ärzte sein sollte, den Patienten eine Art „roten Faden“ für die nützliche Informationsgewinnung über Kopfschmerzen zu geben. Diesen Gedanken verfolgen auch die Autoren einer Arbeit, die sich mit Vor- und Nachteilen der Internetsuche zu medizinischen Themen beschäftigt: der behandelnde Arzt solle Empfehlungen von Internetseiten aussprechen, auf denen seine Patienten gezielt nach weiterführenden Informationen suchen können (Akerkar und Bichile 2004). Ein Problem stellt nämlich auch das Finden und Aufsuchen von qualitativ guten Internetseiten dar, da eine Vielzahl von nur mittelmäßigen Seiten existiert (Peterlin et al. 2008). 39 \% der Patienten, die vor einer Arztkonsultation Informationen im Internet über ihre Beschwerden suchten, informierten sich im Hinblick auf die letztendliche Diagnose über die falsche Erkrankung oder zogen falsche Schlüsse aus den von innen gefundenen Informationen (Larner 2006). Allerdings habe keiner der Patienten hierdurch einen Nachteil oder gar Schaden erlitten.

Andere Quellen wiederum sehen die Entwicklung in den letzten Jahren hin zum informierten Patienten als überaus positiv. Das Internet habe die Möglichkeit, eine wirkliche Partnerschaft zwischen Arzt und Patient aufzubauen und somit die Patientenversorgung zu verbessern (Bauer 2002). Es sei die Basis einer neuen Art der Beziehung zwischen Patient und Arzt, die nun mehr auf das Miteinander aufbaue. Der Patient sei durch seine Informationen mehr in der Lage, aktiv in das Geschehen, sprich in die Therapie miteinzugreifen und diese mitzugestalten (Pemberton und Goldblatt 1998). Allerdings wird auch hier auf das Problem der ungefilterten Information hingewiesen. Außerdem sei es möglich, dass Ärzte sich aufgrund des besseren Wissensstandes ihrer Patienten unter Druck gesetzt fühlen könnten und somit die Arzt-Patienten-Beziehung wiederum negativ beeinflusst sei. Neben diesem Aspekt wird darauf hingewiesen, dass unrelevante und falsche Informationen ebenfalls die Arzt-Patienten-Beziehung verschlechtern und einen negativen Einfluss auf die Therapie haben können (Murray et al. 2003). Viele Ärzte fühlen sich durch das im Internet gewonnene Wissen ihrer Patienten abgeschreckt und entwickeln Vermeidungsstrategien. Allerdings wird der Anteil an gut informierten Patienten weiter steigen und die Ärzte müssen sich Strategien überlegen, wie sie damit angemessen umgehen können. Außerdem kann es zum Problem werden, dass die Lücke zwischen den gut informierten Patienten und denen, die keinen Zugang zum Internet haben, immer größer wird (van Woerkum 2003). Auch wenn Internetinformationen immer noch sehr unregelmäßig in die Beratungen einfließen, sind die Allgemeinärzte diejenigen, die von allen Fachrichtungen am meisten mit der Präsentation von im Internet gewonnenen Informationen zu speziellen Sachverhalten durch die Patienten konfrontiert werden (Malone et al. 2004). Interessant ist, dass hauptsächlich die älteren ausbildenden (Lehr-) Ärzte über 60 
Jahre das Internet häufig dazu nutzen, Fachliteratur zu Erkrankungen ihrer Patienten zu suchen. Allgemein wird das Internet jedoch von $98 \%$ der Lehr-Ärzte mehr oder weniger regelmäßig zum Informationsgewinn genutzt (Carney et al. 2004).

\subsection{Die Rolle des Internets in der medizinischen Betreuung von Patienten}

\subsubsection{Internetnutzung im medizinischen Praxisalltag}

Viele Patienten stehen einer spezifisch medizinischen Nutzung des Internets sehr offen gegenüber. So würde z. B. ein Großteil der Patienten ihren Arzt per E-Mail kontaktieren, auch wenn noch Lücken hinsichtlich des Datenschutzes bestehen (Sittig et al. 2001). Die E-Mails von Patienten an ihre behandelnden Ärzte haben meistens neu aufgetretene Symptome oder Fragen nach aktuellen Laborwerten zum Thema.

Schätzungsweise 60 - $70 \%$ der Ärzte in den Industrieländern haben Zugang zum Internet, wobei die Nutzung im Praxisalltag weiterhin zunimmt (Masters 2008). Vor allem jüngere und weibliche Ärzte nutzen das Internet, um Informationen über spezielle, patientenbezogene Themen zu erhalten (Bennett et al. 2006). Rund ein Viertel der praktizierenden Ärzte gibt an, bereits Kontakt mit Patienten per E-Mail gehabt zu haben (Delbanco und Sands 2004). Eine Kommunikation zwischen Arzt und Patient per E-Mail kann vor allem bei der Betreuung von chronisch Kranken unterstützend eingesetzt werden, da so die kontinuierliche Betreuung besser gewährleistet werden kann und der Arzt in der Lage ist, weniger dringende Anfragen flexibel zu beantworten (Patt et al. 2003). Der Zeitfaktor spielt hierbei eine entscheidende Rolle (Houston et al. 2003).

Der Zeitaufwand macht auf Seiten der Ärzte aber auch einen großen Teil der Vorbehalte gegenüber der Nutzung des Internets innerhalb der Arzt-Patienten-Kommunikation aus. Zum einen wird die Mehrarbeit, die durch eine derartige zusätzliche Kommunikationsmöglichkeit auf die Ärzte zukommt, negativ bewertet (Neville et al. 2004 a). Zum anderen werden Lücken hinsichtlich der rechtlichen Situation und die fragliche angemessene Benutzung von derartigen E-Mails angeführt (Houston et al. 2003). Hierzu fanden White et al. in einer Studie allerdings heraus, dass die an Ärzte abgeschickten Patientenanfragen medizinisch relevant und präzise gestellt waren und somit Sorgen von Seiten der Ärzte um die Relevanz der angesprochenen Themen unbegründet seien (2004).

Trotz dieser angeführten Einschränkungen sind diejenigen Ärzte, welche bereits Patientenkontakt über das Internet hatten, gegenüber dieser Art der Konsultation jedoch durchaus positiv gestimmt. Sie seien durch den virtuellen Patientenkontakt dahingehend gefor- 
dert, mit unbekannten Patienten zu kommunizieren, ohne diese persönlich in ihrer Praxis zu sehen oder zu untersuchen (Umefjord et al. 2004). Damit die Einbeziehung des Internets die alltägliche Arbeit in der Praxis erleichtere und nicht behindere, seien allerdings strukturelle Veränderungen des Praxisablaufs nötig, um dies reibungslos gewährleisten zu können (Bodenheimer und Grumbach 2003).

\subsubsection{Gründe von Patienten zur Internetnutzung für medizinische Zwecke}

Die häufigsten Gründe, das Internet aufzusuchen, sind vor allem Informationen über das eigene Befinden, Therapien und Symptome zu bekommen und Rat zu Therapievorschlägen oder spezifischen Symptomen zu erhalten (Shuyler und Knight 2003). Eysenbach und Diepgen untersuchten E-Mails von Patienten mit hauptsächlich dermatologischen Problemen (1999). Es zeigte sich, dass die ratsuchenden Patienten größtenteils an chronischen Erkrankungen litten (81 \%) und eine zweite Meinung einholen wollten. $17 \%$ berichteten über schlechte Erfahrungen mit anderen Ärzten. Die Autoren warnen davor, dass der E-Mail-Kontakt zu Ärzten von chronisch kranken und enttäuschten Patienten überbeansprucht werden könnte, da diese Patienten verzweifelt nach zusätzlichen Informationen über ihre Krankheit suchten. Andererseits steht auch der geringe Zeitaufwand im Vordergrund (Umefjord et al. 2003). 52 \% der 2207 befragten Patienten nannten dies als Hauptgrund, online eine Frage an einen Arzt in einem Internetforum zu stellen. An zweiter und dritter Stelle folgten die Anonymität des Internets bzw. der Zeitmangel des eigenen behandelnden Arztes. Die Patienten, die Internetsprechstunden nutzen, gehören zumeist eher der jüngeren Generation an (Ramos Martinez et al. 2004). 


\section{Fragestellung und Ziel}

In der Literatur finden sich verschiedene Arbeiten, die die Internetnutzung von Patienten oder ihre Zufriedenheit mit Hausärzten darstellen. Jedoch wurden hierbei die Themen der hausärztlichen Versorgung, Internetnutzung und Zufriedenheit von Kopfschmerzpatienten mit ihrer ärztlichen Versorgung getrennt voneinander untersucht. In der vorliegenden Arbeit sollen diese Aspekte miteinander in Verbindung gebracht werden, um mögliche Abhängigkeiten und Zusammenhänge zu erkennen.

Bei der Entwicklung der Studie stellte sich die Frage, ob Kopfschmerzpatienten das Internet (und im Speziellen den untersuchten Kopfschmerz-Expertenrat) als Ergänzung zum ärztlichen Besuch oder sogar als Ersatz desselben sehen und hier die Beratung und Antworten bekommen, die sie unter Umständen bei ihrem eigenen Arzt vermissen. Außerdem ergab sich die Frage, ob die Nutzer möglicherweise sehr fordernde und anspruchsvolle Patienten darstellten, die in der normalen Hausarztversorgung unter Umständen nicht für ihre Bedürfnisse ausreichend versorgt werden konnten.

Ziel der vorliegenden Studie war es, die Beweggründe von Kopfschmerzpatienten zu explorieren, die einen Expertenrat im Internet besuchten und in diesem Forum Fragen an einen als Experten fungierenden Allgemeinmediziner stellten. Hierzu wurden die Nutzer des Expertenrats mittels eines Internet-Fragebogens zu ihren persönlichen Gründen, den Expertenrat zu besuchen, befragt und gebeten, ihre Erfahrungen mit den jeweiligen behandelnden Ärzten zu bewerten. Zusätzlich wurden sämtliche an den Expertenrat gerichteten Anfragen inhaltsanalytisch untersucht. Dabei wurden neben statistischen Daten wie Alter des Fragestellers oder Länge der Anfrage vor allem die jeweils angesprochenen Themen festgehalten.

Eine wesentliche Arbeitshypothese war daher, dass bei den betroffenen Kopfschmerzpatienten eine hohe Arztverdrossenheit und Unzufriedenheit mit der jeweiligen Behandlung der Kopfschmerzen herrschte. Kopfschmerzpatienten oder auch chronische Schmerzpatienten allgemein stellen nach wie vor eine große Herausforderung für die behandelnden Ärzte dar, vor allem in Zeiten der Budgetierung und des straffen Zeitmanagements einer Praxis (Manzoni und Torelli 2007). Die Behandlung dieser Patienten ist oftmals sehr zeitaufwendig und erfordert von den Ärzten eine hohe Bereitschaft, sich auf den Patienten und seine Beschwerden, Sorgen und Ängste einzulassen. Außerdem ist die Therapie von chronischen Kopfschmerzen ein „dynamischer Prozess“ (Mueller 2007), da sich die Kopfschmerzen im Verlauf der Behandlung häufig verändern und die Therapie stetig angepasst werden muss. 


\section{Methoden}

Um die Fragestellung möglichst umfassend bearbeiten zu können, wurden eine quantitative und eine qualitative Methode miteinander kombiniert. So wurde

1. ein Fragebogen erstellt, der den Expertenrat-Nutzern nach Abschicken einer Anfrage präsentiert wurde und

2. die Gesamtzahl der gespeicherten Anfragen seit Bestehen des Expertenrats in seiner zum Zeitpunkt der Erhebung aktuellen äußeren Form qualitativ untersucht $(n=894)$.

Mithilfe dieser zwei Methoden konnten zum einen die Gründe der Nutzer analysiert und zum anderen die Themen der Anfragen näher untersucht werden. Dies war wichtig, um die Dringlichkeit und Ernsthaftigkeit der Fragen beurteilen zu können. Nur so konnte herausgefiltert werden, welche Bedeutung der Expertenrat für die Nutzer hinsichtlich des Umgangs mit ihren gesundheitlichen Problemen hat.

\subsection{Online-Befragung}

\subsubsection{Methodische Anlage der Untersuchung}

In einem Erhebungszeitraum von neun Monaten und zwei Wochen (vom 20.07.2005 bis zum 04.05.2006) wurden Nutzer des Internet-Expertenrats „Kopfschmerz“ zu ihrer Kopfschmerzsymptomatik, den Gründen für die Nutzung des Expertenrats und die damit verbundenen Erwartungen befragt (cross sectional survey). Des Weiteren sollte festgestellt werden, inwieweit Defizite in der ambulanten Versorgung dem Internetbesuch zugrunde liegen.

\subsubsection{Genehmigung durch die Ethik-Kommission}

Die Studie wurde unter der Antragsnummer 26/7/05 am 15.07.2005 nach Erfüllung spezieller Auflagen von der Ethikkommission der Medizinischen Fakultät der Universität Göttingen genehmigt. So wurde das Anschreiben dahingehend verändert, dass die teilnehmenden Nutzer durch aktives Klicken auf einen Link zum Fragebogen weitergeleitet wurden (informed consent). 


\subsubsection{Teilnehmende Nutzer}

Es wurden alle Nutzer des Internet-Expertenrats „Kopfschmerz" in die Studie eingeschlossen, die vor Ausfüllen des Fragebogens eine Anfrage an den Expertenrat geschickt hatten und in den letzten sechs Monaten von Kopfschmerzen betroffen waren.

\subsubsection{Der Expertenrat}

Das in dieser Arbeit untersuchte Internetforum "Expertenrat Migräne und Kopfschmerz" ist auf der „Lifeline“-Homepage der Business Solutions Medicine Online (BSMO), ein Unternehmen von Springer Science + Business Media und der DGN Services GmbH, zu finden und hat sich zur Aufgabe gemacht, Fragen von Internetnutzern zum Thema „Kopfschmerz" zu beantworten. Als Experte fungiert hierbei ein Facharzt für Allgemeinmedizin, Neurologie und Kinder- und Jugendpsychiatrie, der die gestellten Fragen persönlich beantwortet.

Die Nutzer des Expertenrats geben ihre Fragen nach Akzeptieren der Nutzungsbedingungen in ein Onlineformular ein. Eine Anmeldung mit Benutzernamen und Kennwort ist nicht erforderlich, die Angaben zum Namen und der eigenen E-Mail-Adresse sind freiwillig.

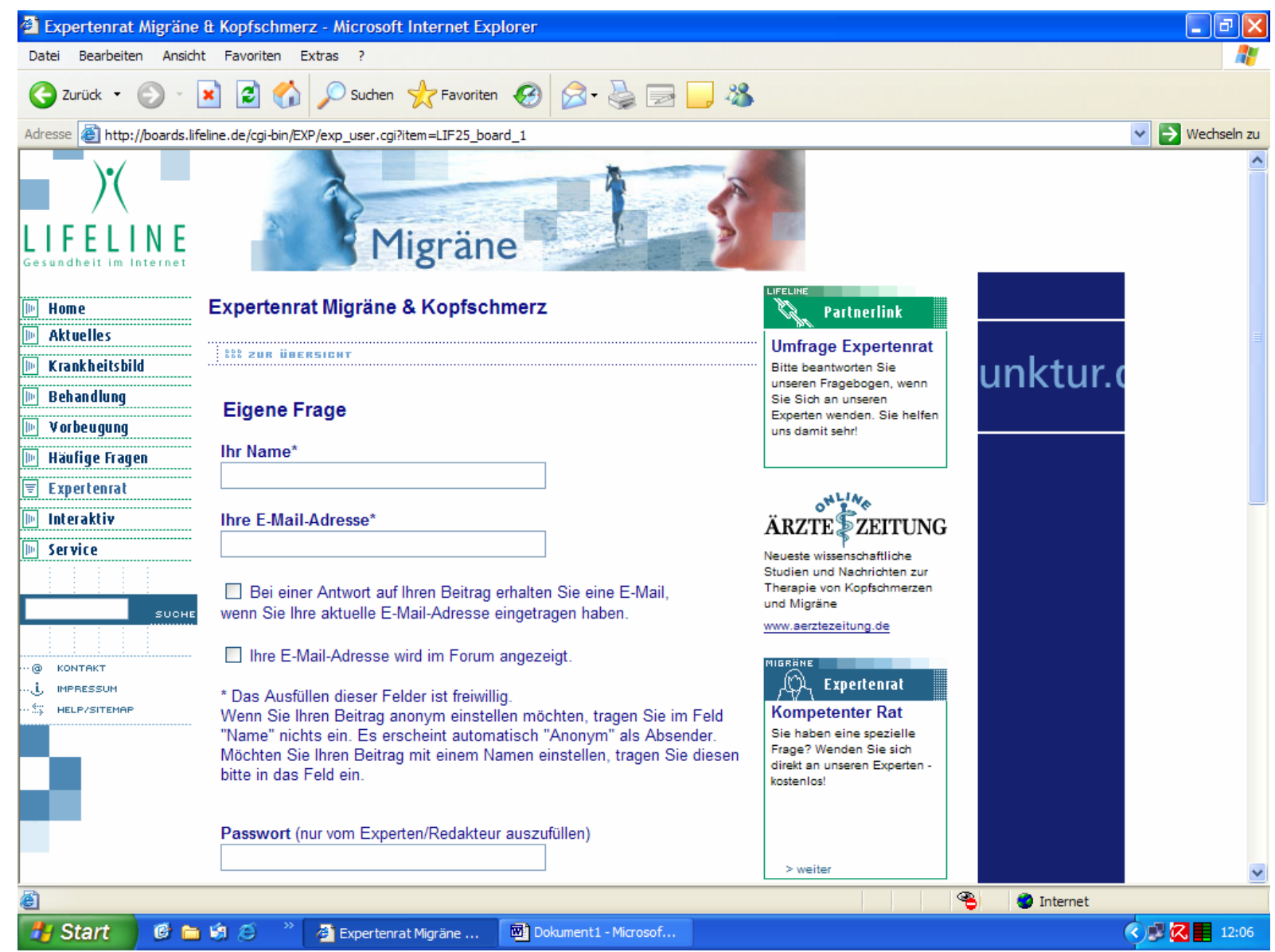

Abbildung 1: Eingabemaske des Expertenrats, Teil 1 


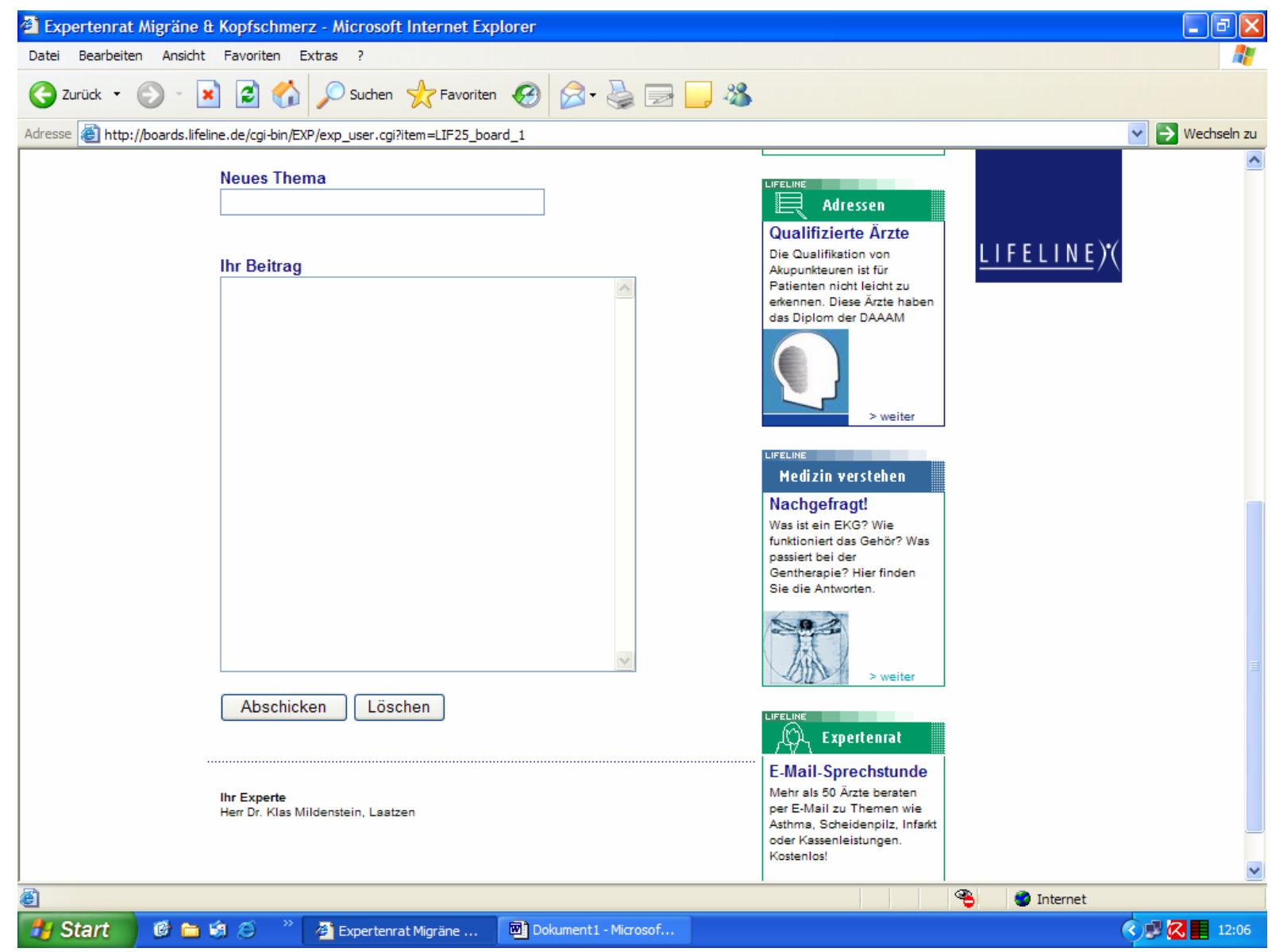

Abbildung 2: Eingabemaske des Expertenrats, Teil 2

Die Fragen und jeweiligen Antworten werden nach Erscheinungsdatum sortiert auf der Internetseite des Expertenrats gespeichert. Die Antworten des Experten sind hierbei den dazugehörigen Fragen unmittelbar zugeordnet. Alle Fragen und Antworten sind für jeden Besucher der Seite einsehbar. Nicht nur der Experte kann auf die Fragen antworten, auch andere Nutzer können schriftlich auf die einzelnen Anfragen reagieren und müssen sich auch hierfür nicht namentlich registrieren. 


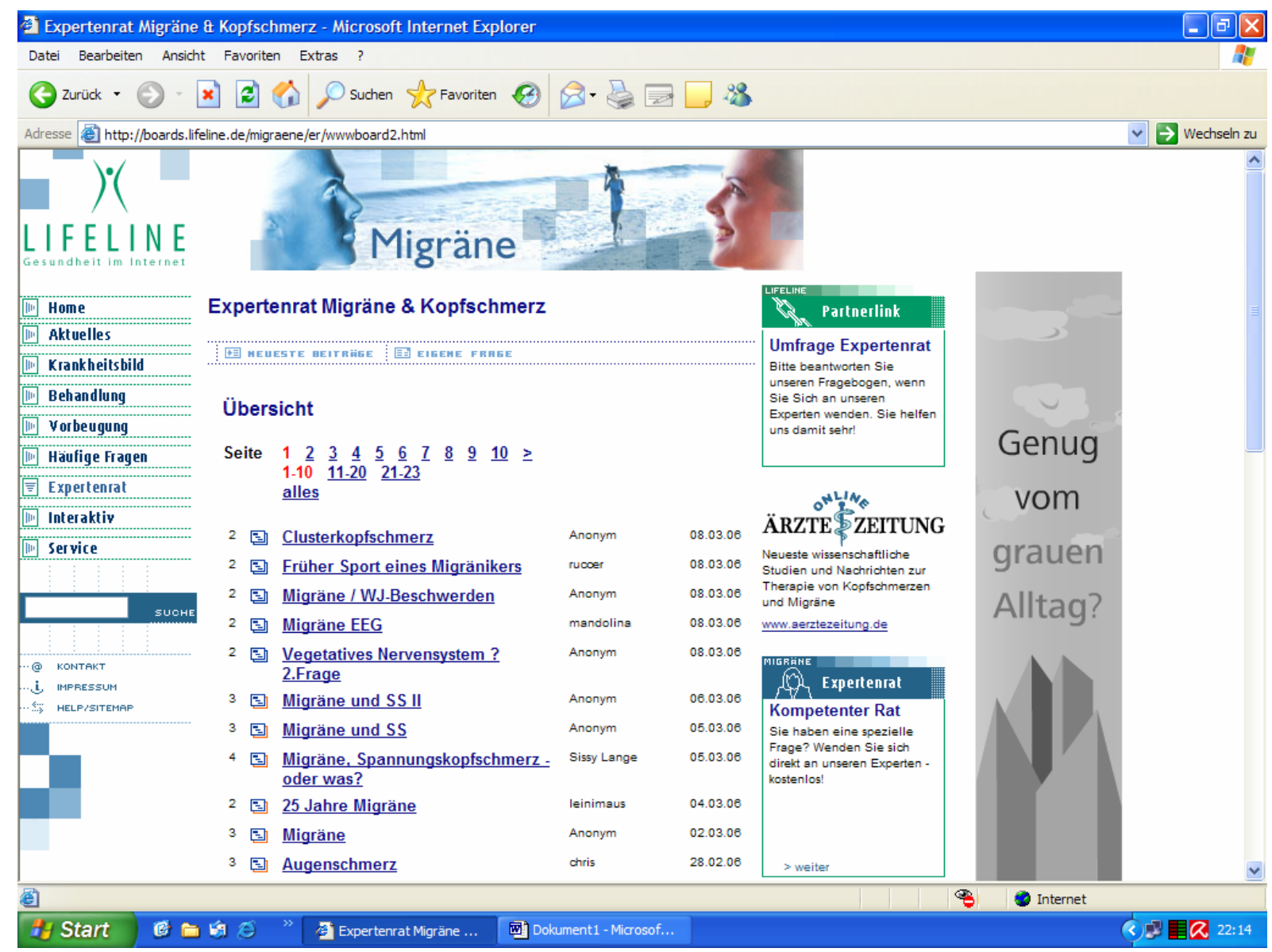

Abbildung 3: Die Anfragen auf der Expertenratseite

\subsubsection{Der Experte}

Der Allgemeinmediziner Herr Dr. med. Klas Mildenstein betreut das Forum seit dem Frühjahr 2002. Regelmäßig beantwortet er die an inn gestellten Fragen der ExpertenratNutzer. Er ist Facharzt für Allgemeinmedizin, Neurologie und Kinder- und Jugendpsychiatrie mit den Zusatzbezeichnungen der speziellen Schmerztherapie und der Chirotherapie. Zudem ist er Psychoanalytiker. Seit 1985 ist er in eigener Praxis in Laatzen als niedergelassener Allgemeinarzt tätig. Seine Praxis ist seit 1998 Schwerpunktpraxis für Diabetologie. Ferner ist er seit 1988 Lehrbeauftragter für Allgemeinmedizin an der Medizinischen Hochschule Hannover. Seine Arbeitsschwerpunkte sind Ernährungsmedizin, Schmerztherapie, Patientenseminare und die medizinische Lehre.

\subsubsection{Fragebogen}

Zur Online-Befragung der Expertenrat-Nutzer wurden zwei Fragebögen miteinander kombiniert. Dabei wurde ein selbst entworfener Fragebogen an den Anfang gestellt. Daran schloss sich der Kieler Kopfschmerzfragebogen von Prof. Hartmut Göbel aus Kiel fließend an. Am Schluss folgten soziodemographische Fragen zu Geschlecht, Staatsangehörigkeit etc. Das Design des Fragebogens wurde betont schlicht gehalten, um die Nutzer nicht 
von der Beantwortung der Fragen abzulenken. Die Online-Präsentation des InternetFragebogens ist in Abbildung 4 dargestellt.

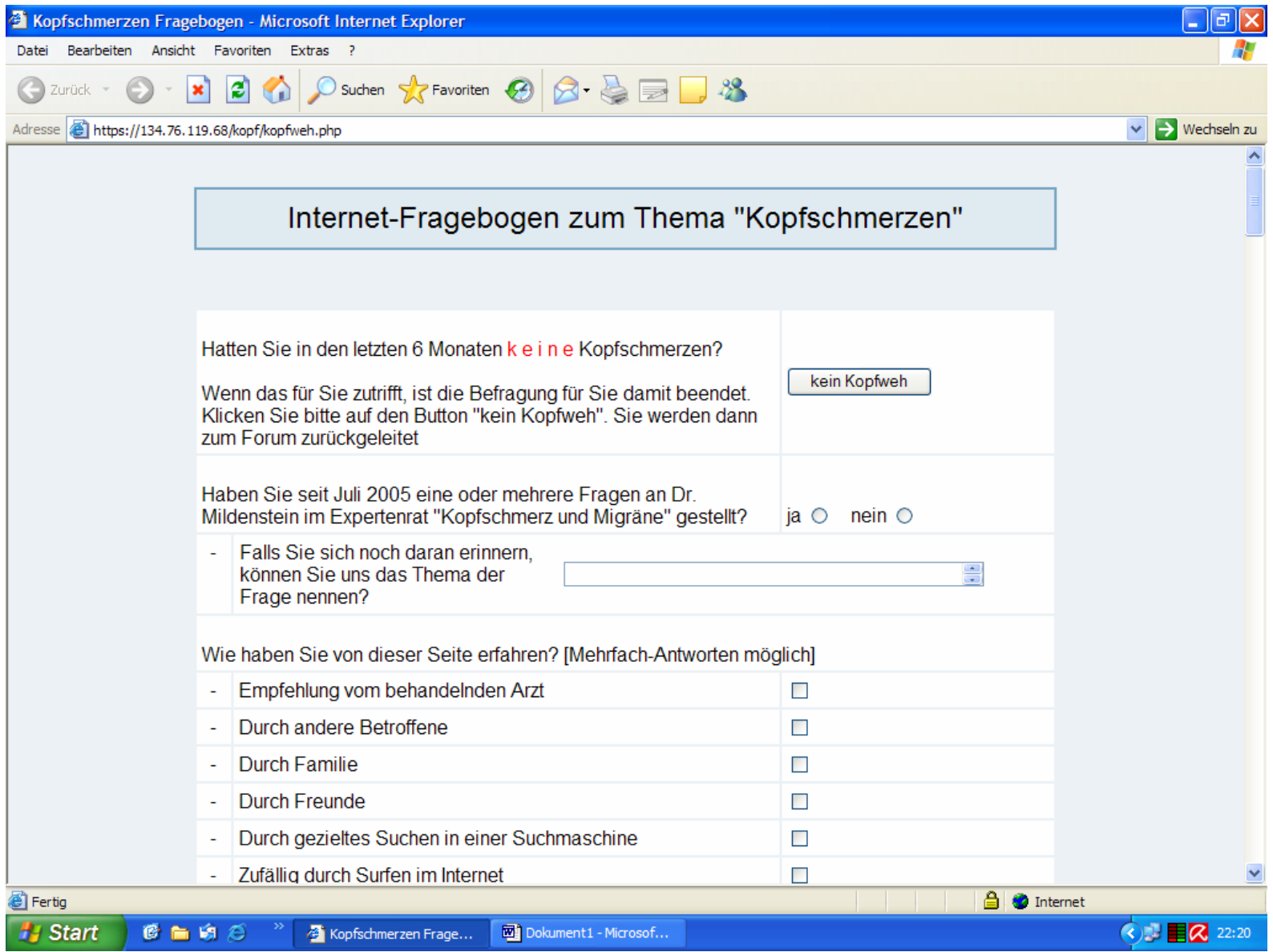

Abbildung 4: Online-Präsentation des Internet-Fragebogens

\section{Selbst entwickelter Fragebogen}

\section{Entwicklung}

Es wurde ein standardisierter Fragebogen entworfen, um die Nutzer nach ihrer Motivation zur Nutzung des Expertenrats und seinen Beratungsmöglichkeiten zu fragen. Der Fragebogen wurde innerhalb der Abteilung Allgemeinmedizin der Universität Göttingen diskutiert, sowohl im abteilungsinternen Doktorandenseminar, in dem die Studie in der Planungsphase vorgestellt wurde, als auch in einer kleineren Diskussionsrunde mit verschiedenen Mitarbeitern der Abteilung.

\section{$\underline{\text { Aufbau }}$}

Beim Aufbau und der Gliederung des Fragebogens wurde auf die gute Verständlichkeit und eindeutige Formulierung der Fragen geachtet. Es sollten beim Ausfüllen keine Ver- 
ständnisfragen auftreten, um eine präzise Bearbeitung der Fragen durch die ExpertenratNutzer zu gewährleisten. Dabei wurden mehrere Fragetypen verwendet:

- $\quad$ dichotome Fragen, mit ,ja“ oder „nein“ zu beantworten, z. B.:

Haben Sie schon einmal andere Internetsprechstunden genutzt?

$$
\text { ja nein } \square
$$

- Mehrfachantwort-Fragen, z. B.:

Wie oft nutzen Sie das Internet, um Informationen zu Kopfschmerzen zu bekommen?

- Täglich

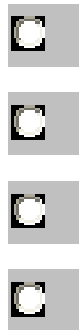

- Wöchentlich

- Monatlich

- Weniger als zwölf Mal pro Jahr

- $\quad$ offene Fragen mit Freitextfeldern, z. B.:

Welche zusätzlichen Erkrankungen außer den Kopfschmerzen sind bei Ihnen bekannt?

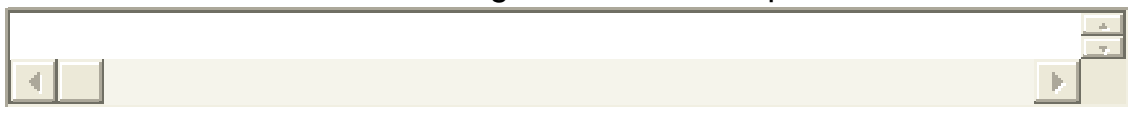

- $\quad$ Fragen mit Antwortmöglichkeiten in Form von Likert-Skalen, z. B.:

\begin{tabular}{lccccc} 
Fühlen Sie sich mit Ihren Beschwerden ernst genommen? & $\begin{array}{c}\text { überhaupt } \\
\text { nicht ernst } \\
\text { genommen }\end{array}$ & & & $\begin{array}{c}\text { sehr ernst } \\
\text { genommen }\end{array}$ \\
- Durch Familie & 1 & 2 & 3 & 4 & 5 \\
- Durch enge Freunde & $\mathbf{C}$ & $\mathbf{C}$ & $\mathbf{C}$ & $\mathbf{C}$ & $\mathbf{C}$ \\
- Durch Bekannte & $\mathbf{C}$ & $\mathbf{C}$ & $\mathbf{C}$ & $\mathbf{C}$ & $\mathbf{C}$ \\
- Durch Arbeitskollegen & $\mathbf{C}$ & $\mathbf{C}$ & $\mathbf{C}$ & $\mathbf{C}$ & $\mathbf{C}$ \\
- Durch den behandelnden Arzt & $\mathbf{C}$ & $\mathbf{C}$ & $\mathbf{C}$ & $\mathbf{C}$ & $\mathbf{C}$ \\
\hline
\end{tabular}


- $\quad$ Folgefragen, die sich auf vorherige Fragen beziehen, z. B.:

Wenn Sie nicht zufrieden sind, was stört Sie bzw. was fehlt Ihnen (bitte geben Sie an, auf welchen Arzt sich Ihre Antwort bezieht: Hausarzt, Neurologe, Augenarzt etc.)?

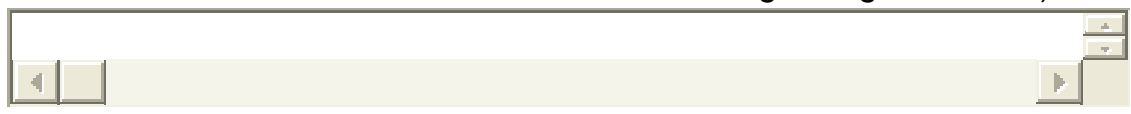

Zunächst erfolgte eine Selektion der Teilnehmer, da nur Nutzer antworten sollten, die in den letzten sechs Monaten über Kopfschmerzen berichten konnten. Dies wurde mit einer Ausschlussfrage sichergestellt:

Hatten Sie in den letzten sechs Monaten k e i n e Kopfschmerzen?

Wenn das für Sie zutrifft, ist die Befragung für Sie damit beendet. Klicken Sie bitte auf den Button "kein Kopfweh". Sie werden dann zum Forum zurückgeleitet

Im Anschluss folgten dann zunächst allgemeine Fragen über die individuelle Nutzung des Expertenrats und das Internetverhalten der Nutzer. Danach wurden Fragen zum Arztverhalten des jeweiligen Nutzers gestellt, wobei auch die Zufriedenheit mit den unterschiedlichen Fachärzten berücksichtigt wurde. Hierbei wurde auch gefragt, inwieweit das Internet mit seinen Beratungsmöglichkeiten in den Augen der Betroffenen Defizite ausgleichen könne. Fragen zur individuellen Behandlung der Kopfschmerzen und anderen Erkrankungen wurden ebenso gestellt wie die Frage, inwieweit sich Betroffene durch behandelnde Ärzte, Familie, Freunde und Bekannte ernst genommen fühlten.

\section{Fragebogen zur Differenzierung der Kopfschmerzen}

Der in dieser Studie verwendete Kieler Kopfschmerzfragebogen wurde von Prof. Hartmut Göbel aus Kiel entwickelt und 1994 erstmals publiziert. Er erlaubt die Differenzierung zwischen Migräne und episodischen bzw. chronischen Kopfschmerzen vom Spannungstyp und wurde auf Grundlage der Kriterien der International Headache Society erstellt (Göbel 1994). Er ist auf zahlreichen Internetseiten frei verfügbar (zum Beispiel auf „www.aspirin.de/service/kopfschmerz-fragebogen.html" oder auf „www.kopfschmerzen.de/Service/Kopfschmerzfragebogen/"). Der standardisierte und gut validierte Fragebogen besteht aus 26 Fragen und einem Auswertungsbogen. Letzterer wurde in der Online-Version des Fragebogens auf der Expertenrat-Homepage weggelassen, da die Auswertung den einzelnen Nutzern nicht zugänglich sein sollte. Die Fragen beziehen sich präzise auf die möglichen Symptome und Begleiterscheinungen, die während eines Kopfschmerzanfalls auftreten können. Von der Gliederung her ist der Fragebogen in zwei Teile geteilt. Jeder Teil enthält 13 dichotome Fragen, die jeweils mit ,ja“ 
oder „nein“ zu beantworten sind. Der erste Teil fragt nach Symptomen, die mit einer Migräne einhergehen können, im zweiten Teil geht es um die Kopfschmerzen vom Spannungstyp. Am Anfang eines jeden Teils steht eine Sondierungsfrage, die sich auf die Hauptsymptome der jeweiligen Kopfschmerzform bezieht:

1. Teil: Treten bei Ihnen Kopfschmerzen auf, die so oder ähnlich aussehen?

- Dauer ohne Behandlung: 4 bis 72 Stunden

- anfallsweises Auftreten,

- zwischen den Anfällen keine Kopfschmerzen

- einseitiges Auftreten

- pochender, pulsierender oder hämmernder Schmerz

- $\quad$ Übelkeit, Erbrechen, Lärm- oder Lichtempfindlichkeit können den Schmerz begleiten

2. Teil: Treten bei Ihnen Kopfschmerzen auf, die man wie folgt beschreiben kann?

- Dauer ohne Behandlung: 30 Minuten bis 7 Tage

- beidseitiges Auftreten

- $\quad$ kann anfallsweise oder täglich auftreten

- drückender, ziehender, dumpfer Schmerz

- $\quad$ kein Erbrechen oder starke Übelkeit

Bei Verneinung dieser Frage wird die ausfüllende Person zum zweiten Teil des Fragebogens geleitet bzw. die Befragung für beendet erklärt:

1. Teil: Falls bei Ihnen solche oder ähnliche Kopfschmerzen auftreten, beantworten Sie bitte die folgenden Fragen.

Treten solche Kopfschmerzen bei Ihnen nicht auf, setzen Sie bitte die Beantwortung bei der Frage 13 fort.

2. Teil: Falls bei Ihnen solche oder ähnliche Kopfschmerzen auftreten, beantworten Sie bitte die folgenden Fragen.

Treten solche Kopfschmerzen bei Ihnen nicht auf, ist die Befragung abgeschlossen.

Anhand des ausgefüllten Fragebogens kann mithilfe des Auswertungsbogens differenziert werden, um welchen Kopfschmerztyp es sich bei dem jeweiligen Betroffenen handelt. Hierbei kann jedoch nur zwischen Migräne, episodischem Kopfschmerz und Spannungskopfschmerz unterschieden werden, andere Kopfschmerzformen werden nicht berücksichtigt. Selbstverständlich kann der Fragebogen nicht eine eingehende körperliche Untersuchung ersetzen, er kann jedoch Anhaltspunkte für eine präzise Anamnese geben 
und nach Ausschluss von sekundären Kopfschmerzformen zur Diagnosestellung herangezogen werden.

\subsubsection{Durchführung der Untersuchung}

\section{Pilotierungsphase}

Auf eine Pilotierungsphase wurde bewusst verzichtet, da der Fragebogen einen relativ langen Entwicklungsprozess durchlaufen hatte und sowohl im Doktorandenseminar der Abteilung Allgemeinmedizin als auch in einer kleineren Runde mit Mitarbeitern der Abteilung diskutiert und bearbeitet wurde. Außerdem waren aufgrund der inhaltlichen Struktur und des Aufbaus der Expertenrat-Homepage technische Schwierigkeiten bei der Umsetzung einer Pilotierung vorhersehbar.

\section{Anschreiben}

Der Fragebogen wurde am 20.07.2005 online geschaltet und konnte ab diesem Zeitpunkt von den Nutzern des Expertenrats bearbeitet und abgeschickt werden. Nach Abschicken einer neuen Frage an den Experten öffnete sich zunächst ein neues Fenster mit einem Anschreiben.

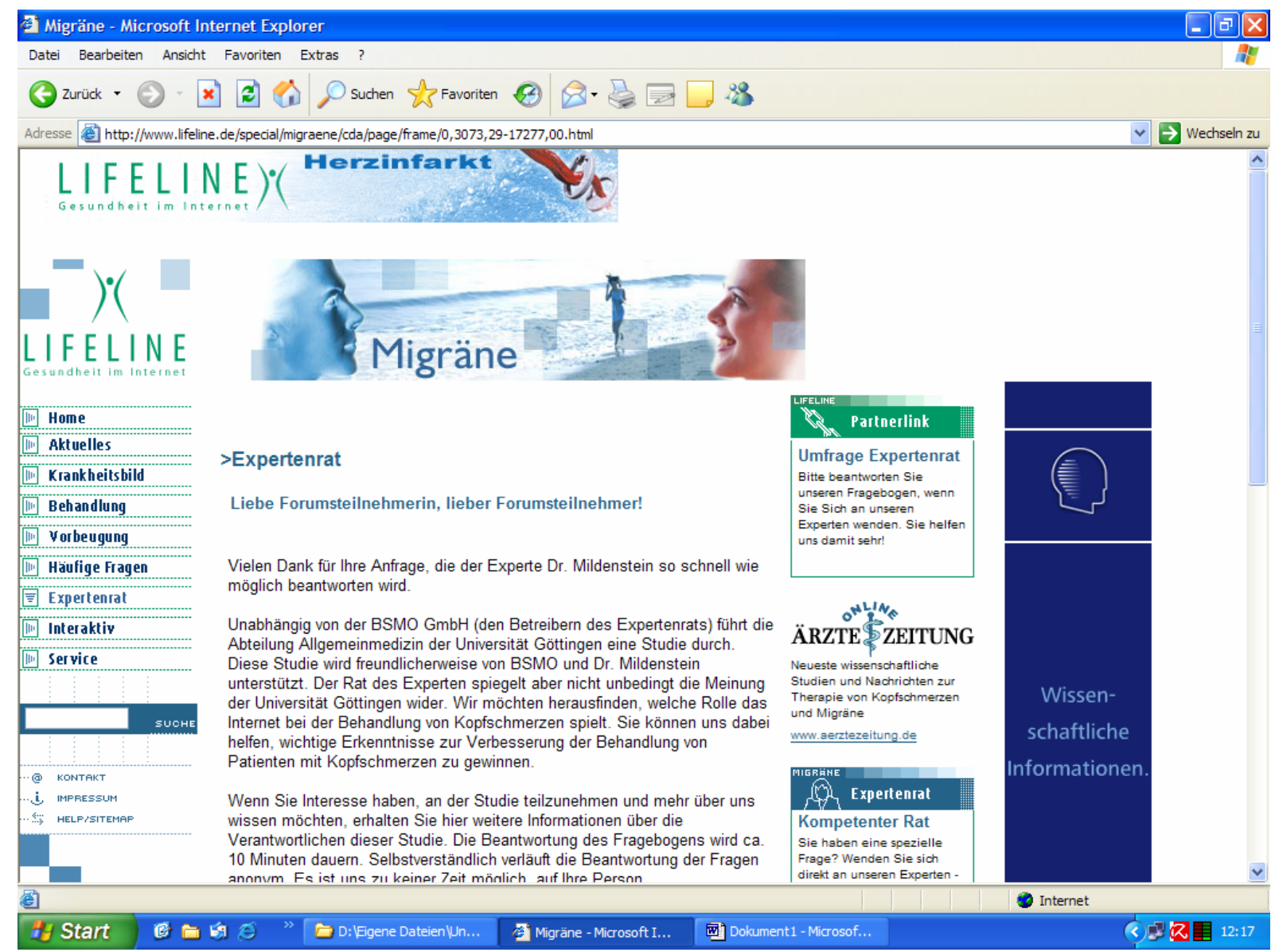

Abbildung 5: Aufruf auf der Expertenrat-Homepage 
In diesem Aufruf, der in dieser Form von der Ethikkommission genehmigt wurde, wurde die Studie kurz vorgestellt und die Nutzer gebeten, daran teilzunehmen. Dabei wurde darauf hingewiesen, dass die Teilnahme an der Umfrage vollständig anonym war und die Beantwortung der Anfrage durch den Experten davon unabhängig ablief. Außerdem wurde die strikte Trennung zwischen Studiendurchführung durch die Abteilung Allgemeinmedizin der Universität Göttingen und der Betreibung des Expertenrats durch die Gesellschaft BSMO hervorgehoben. Erst nach Klicken auf einen Link am Ende des Textes wurden die Nutzer zum Fragebogen geleitet. Am Schluss des Schreibens wurden E-MailAdresse und Homepage der Abteilung Allgemeinmedizin angegeben, um den Nutzern eine Möglichkeit der weiteren Informationsgewinnung zu geben.

\section{Verarbeitung der Daten}

Die Daten der ausgefüllten Fragebögen wurden nach Abschicken in einer verschlüsselten Internet-Datenbank gespeichert. Es handelte sich hierbei um eine "MySQL“-Datenbank, die Administration der Daten erfolgte durch die Applikation „phpMyAdmin 2.3.2“. Das Abfragen der Daten war nur mit Benutzerkennung und Passwort möglich, welche nur ausgewählten Mitarbeitern der Abteilung Allgemeinmedizin bekannt waren. Ein Zugriff Dritter wurde somit verhindert.

Das Ausfüllen des Fragebogens erfolgte anonym. Ein Rückschluss vom ausgefüllten Fragebogen zur Anfrage des jeweiligen Nutzers konnte zwar anhand der Überprüfung der zeitlichen Übereinstimmung der Dateneinträge erfolgen, jedoch erfolgte auch die Absendung einer Frage an den Expertenrat größtenteils anonym. Wenn ein vollständiger Name angegeben wurde, so geschah dies auf freiwilliger Basis. Die IP-Adressen der Benutzer waren nicht zugänglich und wurden somit auch nicht erfasst.

\section{Zusätzliche Internetanzeige}

Um die anfänglich schleppende Rücklaufquote zu erhöhen, wurde am 01.02.2006 eine Teaserbox auf die Expertenrat-Seite gestellt, die die Nutzer direkt zum Fragebogen führte. Diese Box wurde fest in eine Randspalte der Homepage integriert und war somit auf allen Seiten des Expertenrates zu sehen.

Der Inhalt der Teaserbox lautete:

\section{- „Umfrage Expertenrat}

Bitte beantworten Sie unseren Fragebogen, wenn Sie sich an unseren Experten wenden. Sie helfen uns damit sehr!" 
Durch Klicken auf die Teaserbox wurden die Nutzer zum Fragebogen weitergeleitet.

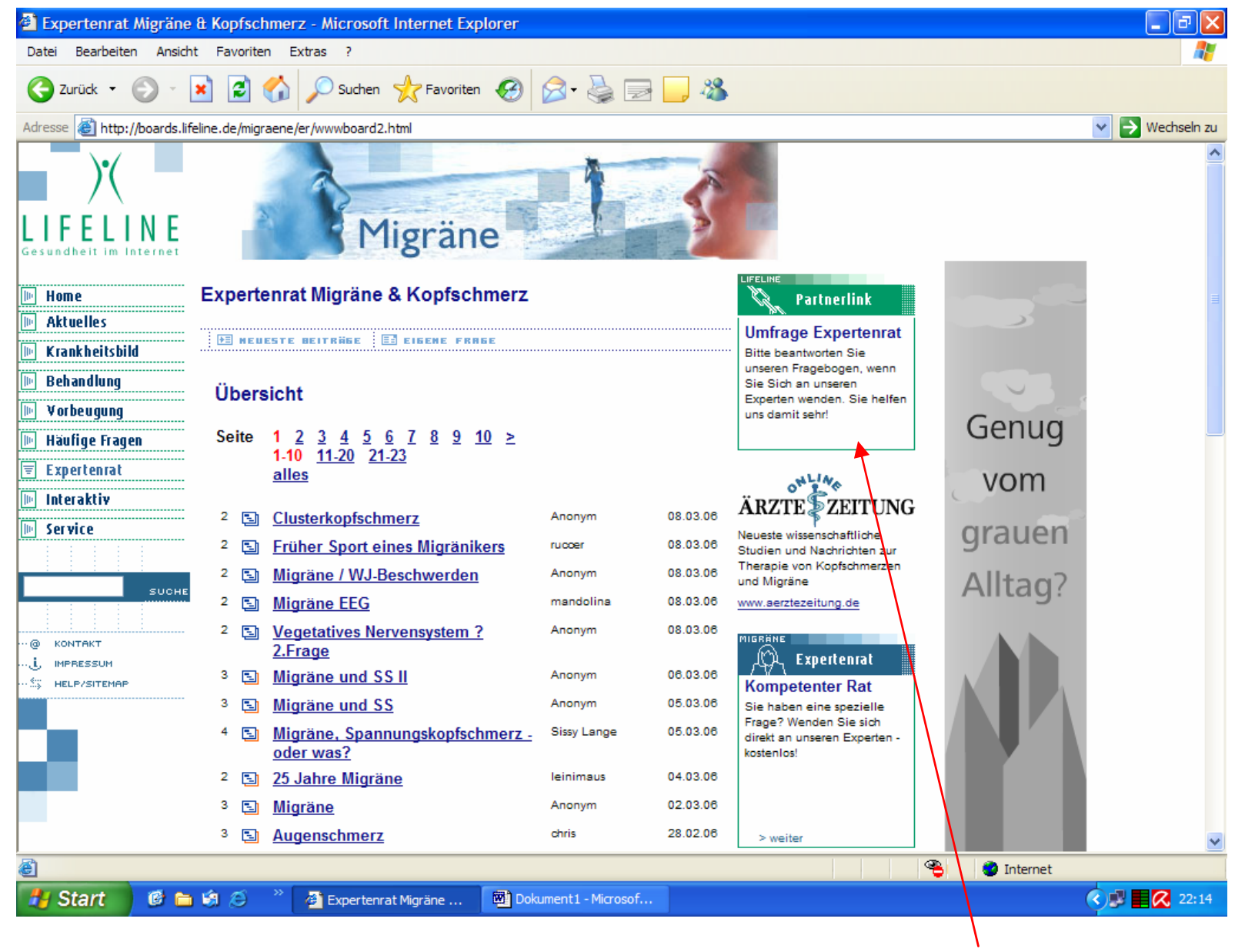

Abbildung 6: Homepage des Expertenrats „Migräne und Kopfschmerz" mit Teaserbox

Um wirklich nur Nutzer in die Studie einzuschließen, die auch eine Anfrage abgeschickt hatten, wurden die Einträge im Expertenrat und der Datenbank des Fragebogens zeitlich miteinander verglichen. Gab es hierbei Übereinstimmungen, so konnte davon ausgegangen werden, dass der Fragebogen-Eintrag und die Anfrage vom selben Nutzer stammten.

Ab dem 15.02.2006 wurde eine veränderte Version des Fragebogens ins Netz gestellt. Dieser Fragebogen enthielt eine zusätzliche Sondierungsfrage, um zu eruieren, ob der jeweilige Nutzer eine Anfrage an den Experten geschickt hatte.

Haben Sie seit Juli 2005 eine oder mehrere Fragen an Dr. Mildenstein im Expertenrat "Kopfschmerz und Migräne" gestellt?

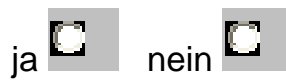

- Falls Sie sich noch daran erinnern, können Sie uns das Thema der Frage nennen?

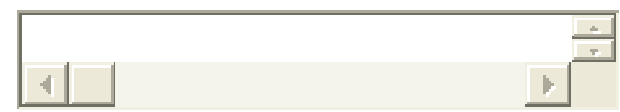


Somit konnte einfacher ermittelt werden, ob der betreffende Nutzer in die Studie eingeschlossen werden konnte oder nicht.

Zusätzlich zu der Teaserbox wurde ab dem 14.02.2006 jeweils eine eigene Antwort mit einem Hinweis auf die Studie und den Fragebogen unter die einzelnen Anfragen gesetzt.

- „Liebe Fragestellerin, lieber Fragesteller!

Wir von der Universität Göttingen, Abteilung Allgemeinmedizin, führen im Moment eine Studie zum Thema „Kopfschmerz" durch und beschättigen uns dabei mit diesem Expertenrat. Sie helfen uns sehr, wenn Sie unseren Fragebogen ausfüllen. Den Link dorthin finden Sie in der Box mit der Überschrift „Umfrage Expertenrat" auf der rechten Seite.

Vielen Dank für Ihre Hilfe!"

Ausgespart wurden hierbei Anfragen, deren Verfasser entweder keine Kopfschmerzen hatte oder den Fragebogen ausgefüllt hatte. Auch dies wurde wieder mit den zeitlichen Übereinstimmungen der Dateneinträge überprüft.

Die eigene Antwort wurde ab dem 22.02.2006 um die Angabe der E-Mail-Adresse von Prof. Martin Scherer ergänzt, um Rückfragen der Benutzer möglich zu machen. Tatsächlich gab es am 25.04.2006 eine Reaktion einer Nutzerin, die den in der Antwort beschriebenen Link auf den Fragebogen nicht auf der Expertenrat-Seite finden konnte. Daher wurde die Antwort ab dem 25.04.2006 dahingehend verändert, dass die Lokalisation der Teaserbox mit dem Link auf der Homepage noch näher beschrieben wurde.

- „Liebe Fragestellerin, lieber Fragesteller!

Wir von der Universität Göttingen, Abteilung Allgemeinmedizin, führen im Moment eine Studie zum Thema „Kopfschmerz" durch und beschäftigen uns dabei mit diesem Expertenrat. Sie helfen uns sehr, wenn Sie unseren Fragebogen ausfüllen. Klicken Sie dafür einfach auf der rechten Seite in der obersten Box „Partnerlink“ auf die Überschrift „Umfrage Expertenrat“.

Sollten Sie noch Fragen haben, schreiben sie bitte an Martin.Scherer@medizin.uni-goettingen.de

Vielen Dank für Ihre Hilfe! 
Mit freundlichen Grüßen,

Sophie Zitterbart und Martin Scherer

\author{
Abt. Allgemeinmedizin Universität Göttingen \\ http://www.allgemeinmedizin.med.uni-goettingen.de/"
}

Außerdem wurden jeweils am 16.02.2006 und 16.03.2006 eigene Anfragen formuliert, die wiederum auf die Studie verwiesen und in denen die Nutzer dazu aufgefordert wurden, den Fragebogen auszufüllen und somit an der Studie teilzunehmen. Der Wortlaut dieser Anfragen glich dem der oben beschriebenen eigenen Antworten.

Ab dem 02.05.2006 schließlich wurden auf der Expertenrat-Homepage von Seiten der Betreiberfirma her Wartungsarbeiten durchgeführt, so dass keine neuen Anfragen möglich waren. Der Fragebogen konnte in dieser Zeit aber noch über die Teaserbox erreicht und ausgefüllt werden. Am 04.05.2006 kam es dann zur vollständigen Umgestaltung des Online-Auftritts des Expertenrats, der in diesem Zuge auch eine neue Internetadresse erhielt. Damit war keine Verlinkung zum Fragebogen mehr vorhanden, so dass die OnlineBefragung für beendet erklärt wurde.

\title{
4.2 Anfragen
}

Als zweite Säule der Methodik wurden alle gespeicherten Anfragen an den Expertenrat seit Beginn der Beratungstätigkeit des Experten bis zur Beendigung der Online-Befragung der Nutzer qualitativ untersucht $(n=894)$. Es wurden demnach alle Anfragen analysiert, die in der Zeit vom 22.04.2002 bis zum 04.05.2006 an den Expertenrat gestellt wurden.

\subsubsection{Kategorien der Anfragenanalyse}

Die Anfragen wurden inhaltsanalytisch ausgewertet, das heißt sie wurden ihrem Inhalt und Aufbau entsprechend in 29 verschiedene Kategorien eingeordnet. Hierbei ging es neben formalen (wie z. B. die Anzahl der Wörter) und soziodemographischen Aspekten (Alter und Geschlecht des Fragestellers) vor allem um die Klassifizierung des Themas und des Inhalts der Anfrage. Hinsichtlich der inhaltlichen Einordnung wurden sämtliche Themen berücksichtigt, die in der Frage angesprochen wurden. Dabei konnten die einzelnen Fragen auch in mehrere Kategorien gleichzeitig einsortiert werden. Die dabei erhaltenen Werte wurden mithilfe des Programms „SPSS“ quantitativ ausgewertet. 


\section{Charakteristika der Anfragen}

- Anfrage-Nummer

Fortlaufende Nummerierung der Anfragen nach Erscheinen auf der ExpertenratHomepage

- Datum der Anfrage

Erstellungsdatum der Anfrage

- $\quad$ Anzahl der Wörter der Anfrage

Wörterzählung mit automatischer Wörterzählung des Programms "Microsoft Word“

- Geschlecht des Fragestellers

Die Zuordnung zu einem Geschlecht erfolgte, wenn möglich, entweder durch direkte Angabe des Fragestellers oder durch Rückschluss auf den angegebenen Namen.

- $\quad$ Alter

Das Alter des Fragestellers wurde, wenn angegeben, berücksichtigt. Hierbei wurden ungefähre Werte gerundet (Angabe „fast 30“ $=>30$, „Mitte 40“ => 45).

- $\quad$ Name

Hierbei wurde unterschieden, ob der Nutzer seinen Namen nicht angegeben hatte oder ob er nur die Initialen oder einen Phantasie-/ Spitznamen, nur den Vornamen mit evtl. Angabe der Initialen des Nachnamens oder den Nachnamen bzw. den vollen Namen angegeben hatte. Der Name konnte entweder im dafür vorgesehenen Namensfeld oder in der Anfrage selbst (z. B. als Verabschiedungsformel) angegeben werden.

- E-Mail

Es wurde vermerkt, ob der Absender der Anfrage seine E-Mail-Adresse angegeben hatte oder nicht. Bis Anfrage Nr. 490 geschah dieses entweder im Fragentext oder als Teil des Namens, später wurde von der Betreiberfirma der Website ein zusätzliches Eingabefeld für die E-Mail-Adresse in die Maske für neue Anfragen eingefügt. 
- Anfrage

Es wurde unterschieden, ob der Nutzer die Frage für sich selbst, für jemanden anderes oder nicht-personenbezogen gestellt hatte.

- $\quad$ Antwort des Experten

Festgehalten wurde, ob der Experte überhaupt auf die Anfrage antwortete, nicht jedoch der Inhalt oder die Ausführlichkeit der Antwort.

- Antwort des Fragestellers auf den Experten

Wenn der Fragesteller auf die Antwort des Experten mit einem schriftlichen Kommentar reagierte, wurde dies in dieser Kategorie festgehalten. Hierbei erfolgte die Identifizierung des jeweiligen Nutzers entweder über die eindeutige Angabe des Namens oder über die direkte Bezugnahme auf die Anfrage innerhalb des Textes.

- Antwort anderer Nutzer

Sofern andere Nutzer auf die Anfrage reagierten, wurde dies hier dokumentiert. Wurden in diesen Antworten neue Anfragen formuliert, wurden diese nicht extra kategorisiert.

- Antwort auf frühere Mails

Wurde Bezug zu früheren Anfragen genommen, so wurde dies hier vermerkt.

- fehlerhafte, unvollständige Mail/ Datenfehler

War die Anfrage fehlerhaft gespeichert und somit nicht vollständig lesbar, wurde dies in dieser Untergruppe dokumentiert. Diese Fragen wurden bei der quantitativen Auswertung nicht berücksichtigt.

\section{Inhaltliche Kategorien der Anfragen}

- $\quad$ Symptombeschreibung

- Aufzählungen von Symptomen und Fragen nach einer möglichen Differentialdiagnose

- Frage nach bestimmten Symptomen (wie z. B. die visuelle Aura bei Migräne)

- Diagnostik, Untersuchungsergebnisse

- Fragen zu weiteren, evtl. nötigen diagnostischen Schritten und zu speziellen, den einzelnen Nutzer betreffenden Untersuchungsergebnissen 
- $\quad$ ögliche Therapien

- Fragen nach möglichen Therapien der individuellen Beschwerden und nach Therapievorschlägen des Experten

- bestimmte Medikamente/ Therapien

- Fragen nach bestimmten Medikamenten oder Medikamentengruppen

- Fragen zu bestimmten (schulmedizinischen) Therapieformen wie z. B. operative Verfahren

- alternative Therapien

- Fragen zu alternativen Therapien (wie z. B. Akupunktur, Entspannungstechniken etc.) und deren Wirksamkeit und Nutzen

- Vorbeugung/ Prophylaxe

- Fragen nach möglicher Vorbeugung von Kopfschmerzanfällen

- Ursachen der Kopfschmerzen

- Fragen zu möglichen Ursachen der Kopfschmerzen (Pathophysiologie, Grunderkrankungen wie z. B. Epstein-Barr-Virus-Infektion, Borreliose)

- Hormonelle Ursachen/ Behandlung

- Fragen zu hormonellen Zusammenhängen (Menstruation, Pilleneinnahme, Menopause, Schilddrüsenhormone etc.)

- Schwangerschaft und Migräne

- Fragen nach Migräne in der Schwangerschaft und Stillzeit (Besonderheiten etc.)

- Triggerfaktoren/ Auslöser/ Verstärker

- Fragen nach möglichen Auslösern und Triggerfaktoren der Kopfschmerzen

- Zusammenhang mit anderen Erkrankungen

- Fragen zu anderen bestimmten Erkrankungen, die mit den Kopfschmerzen in Verbindung stehen könnten

- Fragen zu Risiken von Folgeerkrankungen und/ oder Spätschäden (z. B. Schlaganfall, Aneurysma, Erblindung durch Aura) 
- Fragen zu Erkrankungen, die unter Umständen durch Migräne ausgelöst werden können (z. B. Depressionen)

- Kindermigräne

- Fragen zu Kopfschmerzen (vor allem Migräne) im Kindes- und Jugendalter

- (Fach-) Arzt-/ Therapeutenempfehlung

- Fragen nach speziellen Facharztempfehlungen (Neurologe, HNO, Augenarzt etc.) bzw. nach Empfehlungen von anderen Therapeuten (Physiotherapeut etc.)

- Fragen zu namentlichen Empfehlungen bestimmter Ärzte/ Therapeuten

- Fragen zu Kuraufenthalten

- Hilfe

- Fragen, die die Suche um psychologischen und emotionalen Beistand beinhalteten

- Anfragen, in denen Äußerungen von Angst und Unsicherheit laut wurden

- Schlaf-/ Lebens-/ Essgewohnheiten, Sport/ körperliche Betätigung

- Fragen zu bestimmten Lebensgewohnheiten und deren Nutzen und/ oder Schaden

- Sonstige Anfragen

- Anfragen, die nicht den anderen Kategorien zugeordnet werden konnten

\subsubsection{Datenanalyse}

Die erhobenen Daten wurden in das Auswertungsprogramm „SPSS“ eingegeben und nach Abschluss der Datenerhebung quantitativ ausgewertet. Dabei wurden bei Kategorien wie z. B. "Geschlecht des Fragestellers" und „Angabe der E-Mail-Adresse“ und bei den einzelnen Themen-Untergruppen die Häufigkeiten in Prozent berechnet. Bei den Kategorien „Alter des Fragestellers“ und „Anzahl der Wörter“ wurden Minimum, Maximum, Mittelwert und Standardabweichung (SD) bestimmt. 


\section{Ergebnisse}

\subsection{Online-Befragung}

\subsubsection{Stichprobe}

Der Fragebogen konnte in der Zeit vom 20.07.2005 bis zum 04.05.2006 von den Besuchern des Kopfschmerz-Expertenrats auf der „Lifeline“-Homepage der Gesellschaft Business Solutions Medicine Online (BSMO) ausgefüllt und abgeschickt werden.

Während des Erhebungs-Zeitraums der Online-Umfrage wurden 180 neue Anfragen an den Expertenrat gestellt, 61 Fragesteller klickten in dieser Zeit auf den Link zum Fragebogen. Davon hatten zehn Nutzer keine Kopfschmerzen, die den Fragebogen nicht ausfüllten, da sie durch die Bejahung der Sondierungsfrage (,Hatten Sie in den letzten sechs Monaten keine Kopfschmerzen?") nicht dem für die Studie notwendigen Profil entsprachen. Zwölf weitere Studienteilnehmer hatten seit Juli 2005 keine eigene Frage an den Expertenrat gestellt, so dass sie bei der Auswertung des Fragebogens nicht berücksichtigt wurden.

Somit konnten 39 Fragebögen vollständig für die Analyse verwendet werden.

Tabelle 1: Stichprobenausschöpfung der Online-Befragung

\begin{tabular}{lll}
\hline & Anzahl (n) & Prozent (\%) \\
\hline Neue Anfragen während des Erhebungszeitraums & 180 & 100,0 \\
Abschicken des Fragebogens & 61 & 33,9 \\
Keine Kopfschmerzen & 10 & 5,6 \\
Keine eigene Anfrage & 12 & - \\
Studienteilnehmer & 39 & 21,7 \\
\hline
\end{tabular}

\subsubsection{Stichprobenpopulation der Online-Befragung (soziodemographische Daten)}

Der Großteil der Studienteilnehmer war weiblich $(87,2 \%, n=34)$, nur vier der befragten Nutzer waren männlichen Geschlechts (10,3 \%). Ein/e Nutzer/in gab das Geschlecht nicht an $(2,6 \%)$. 
84,6\% der teilnehmenden Probanden waren deutsche Staatsbürger $(n=33)$, jeweils ein Spanier, ein Österreicher, ein Schweizer und ein Kroate waren vertreten (je 2,6 \%). 5,1 \% der Befragten gaben ihre Staatsangehörigkeit nicht an $(n=2)$.

Von den 39 Studienteilnehmern gaben 25 (64,1 \%) an, mit einem festen Partner zusammenzuleben. 13 (33,3 \%) verneinten diese Frage, einmal wurde sie nicht beantwortet $(2,6$ \%). Durchschnittlich lebten 2,6 Personen in den betreffenden Haushalten (Minimum 1, Maximum 7, SD 1,443).

Das Durchschnittsalter der Stichprobe betrug 33,8 Jahre (Minimum 17, Maximum 57, SD 9,887), wobei einmal keine Angabe zum Alter gemacht wurde.

Tabelle 2: Stichprobenpopulation der Online-Befragung

\begin{tabular}{|c|c|c|c|c|}
\hline & & Anzahl (n) & \multicolumn{2}{|c|}{ Prozent (\%) } \\
\hline \multicolumn{5}{|l|}{ Geschlecht } \\
\hline - Weiblich & & 34 & & \\
\hline - Männlich & & 4 & & \\
\hline - Keine Angabe & & 1 & & \\
\hline \multicolumn{5}{|l|}{ Staatsangehörigkeit } \\
\hline - Deutsch & & 33 & & \\
\hline - Spanisch & & 1 & & \\
\hline - Schweizerisch & & 1 & & \\
\hline - Österreichisch & & 1 & & \\
\hline - Kroatisch & & 1 & & \\
\hline - Keine Angabe & & 2 & & \\
\hline \multicolumn{5}{|l|}{ Zusammenleben mit festem Partner } \\
\hline - Ja & & 25 & & \\
\hline - Nein & & 13 & & \\
\hline \multirow[t]{2}{*}{ - Keine Angabe } & & 1 & & \\
\hline & Mittel & Minimum & Maximum & SD \\
\hline Anzahl der Personen im Haushalt & 2,6 & 1 & 7 & 1,443 \\
\hline Alter der Studienteilnehmer (Jahre) & 33,8 & 17 & 57 & 9,887 \\
\hline
\end{tabular}




\subsubsection{Kontakt zum Expertenrat}

Die Betroffenen gelangten am häufigsten durch gezieltes Suchen mit Hilfe einer InternetSuchmaschine zum Kopfschmerz-Expertenrat $(61,5 \%, n=24) .30,8 \%$ stießen zufällig beim Surfen durch das Internet auf die Homepage $(n=12)$. Ein Teilnehmer gab an, schon früher den Expertenrat aufgesucht zu haben, damals habe er ebenfalls zufällig durch Surfen im Internet die Seite gefunden. Zwei Nutzern (5,1 \%) wurde der Expertenrat von anderen Betroffenen empfohlen. Direkte Empfehlungen der Seite durch Freunde, Familie oder aber den behandelnden Arzt spielten jedoch keine Rolle.

\subsubsection{Internetsprechstunden, Internetverhalten}

38,5 \% der Studienteilnehmer $(n=15)$ hatten bereits früher einmal Internetsprechstunden genutzt, für den Großteil $(56,4 \%, n=22)$ aber war der Kopfschmerz-Expertenrat der erste Kontakt zu dieser elektronischen Form des Arztbesuches.

Durchschnittlich wurde der Expertenrat von den unterschiedlichen Nutzern seit 15,3 Wochen benutzt (Minimum 0, Maximum 99, SD 28,766). Dabei gaben 61,5\% ( $n=24)$ an, Internetsprechstunden weniger als zwölf Mal im Jahr zu besuchen. 15,4 \% $(n=6)$ der Befragten nutzen dieses elektronische Angebot wöchentlich. Vier weitere Teilnehmer $(10,3 \%)$ waren monatliche Nutzer von Internetsprechstunden, nur zwei der Befragten (5,1 \%) besuchten täglich Sprechstunden im Netz.

Bei der Informationsgewinnung zum Thema „Kopfschmerz“ gaben 41,0 \% ( $n=16)$ der Teilnehmer an, weniger als zwölf Mal im Jahr Informationen über Kopfschmerzen im Internet zu suchen. Immerhin 25,6\% $(n=10)$ suchten wöchentlich gezielt nach Kopfschmerz-Themen, während jeweils 15,4\% $(n=6)$ monatlich beziehungsweise täglich danach suchten.

Im Hinblick auf das generelle Internetverhalten der Studienteilnehmer ist festzustellen, dass der Großteil das Internet zur allgemeinen Informationsgewinnung nutzte (79,5\%, n = 31). Die Mehrzahl der Nutzer $(61,5 \%, n=24)$ machte auch Gebrauch vom OnlineBanking. Weitere 53,8 \% ( $n=21)$ kauften online ein, unter anderem auch in OnlineAuktionshäusern. Wichtig schien auch die Nutzung von Foren und Chats zu sein, dies gaben 48,7 \% ( $n=19)$ an. Einen weiteren großen Aspekt der Internet-Nutzung stellte die Reisebuchung dar, die 46,2 \% $(n=18)$ der Befragten wahrnahmen. Weitere Nutzungsgründe waren die Suche nach speziellen Fachinformationen für Studium und Beruf (10,3 $\%, n=4)$, das Versenden und Empfangen von E-Mails $(5,1 \%, n=2)$, sowie Preisvergleiche, Online-Telefonieren und private Hobbyseiten (jeweils 2,6 \%, $\mathrm{n}=1$ ). 


\subsubsection{Medikamente/ Therapie}

Die Betroffenen wurden in dem Fragebogen nach ihrer aktuellen Medikation gefragt. 79,5 $\%(n=31)$ der Studienteilnehmer gaben an, medikamentös gegen ihre Kopfschmerzen vorzugehen. Dabei wurden an erster Stelle der Akuttherapie die Triptane genannt. 46,2 \% der Befragten $(n=18)$ gaben an, diese speziellen Migränemittel bei einem akuten Anfall einzunehmen. Eine weitere wichtige Rolle spielten bei der Therapie die Non-Steroidalen Antirheumatika (NSAR), wobei hier am häufigsten ASS, Paracetamol und Ibuprofen genannt wurden $(35,9 \%, n=14)$. Diese Medikamentengruppe wurde häufig auch als Kombinationspräparat, z. B. mit Metoclopramid oder Koffein, eingenommen. Neben weiteren Akutpräparaten wie Metamizol, Tramadol oder Opioiden wurden außerdem Medikamente zur Anfallsprophylaxe genannt. Dies war vor allem die Gruppe der Betablocker (17,9 \%, n $=7)$.

Tabelle 3: Medikation der Studienteilnehmer

Anzahl (n)

Prozent (\%)

Akutmedikation:

- Triptane

46,2

- NSAR (auch Kombipräparate)

- Antiemetika (Metoclopramid/ Dimenhydrinat)

- Metamizol/ Phenazon

- Benzodiazepine 3

5,1

- Tramadol

- Ergotamin

- Katadolon

- Opioide

Anfallsprophylaxe:

- Betablocker 7

- Antidepressiva

- Magnesium

- Pestwurzpräparat

- Calciumantagonisten

2

- ACE-Hemmer 
Neben der medikamentösen Therapie wurden die Expertenrat-Nutzer auch nach anderen Therapieformen gefragt. Hierbei gaben 35,9 \% $(n=14)$ an, aufgrund der Kopfschmerzen schon einmal in physiotherapeutischer Behandlung gewesen zu sein. 33,3\% ( $n=13)$ berichteten über Therapieversuche mit Massagen, 41,0 \% $(n=16)$ hatten bereits Erfahrungen mit Akupunktur gemacht. Autogenes Training hatten bereits 20,5\% ( $n=8)$ der Betroffenen ausprobiert, 30,8 \% ( $n=12)$ hatten versucht, die Kopfschmerzen mit Entspannungstechniken zu bekämpfen. Immerhin sieben der befragten Nutzer (17,9\%) hatten aufgrund ihrer Kopfschmerz-Problematik bereits psychotherapeutische Hilfe in Anspruch genommen. Drei der Studienteilnehmer (7,7 \%) gaben an, andere Therapieansätze probiert zu haben, darunter die Hydrocolontherapie, Eigenurinbehandlung, Chiropraktik, Neuraltherapie, Osteopathie und die Verwendung eines TENS-Gerätes (TENS = Transkutane elektrische Nervenstimulation) und einer Zahnschiene.

\subsubsection{Krankengeschichte der Studienteilnehmer}

Nach ihrer eigenen Krankengeschichte befragt, gaben 51,3\% $(n=20)$ der Studienteilnehmer an, an weiteren Erkrankungen neben den Kopfschmerzen zu leiden. Hierbei wurden ganz unterschiedliche Krankheiten aufgeführt, so z. B. Allergien, rezidivierende Harnwegsinfekte und Schilddrüsenunterfunktion. Aber auch psychische Erkrankungen wie Depression, Angstzustände und Bulimie wurden genannt. Am häufigsten waren mit 10,3 $\%$ die Schilddrüsenerkrankungen vertreten, hieran waren vier der Befragten erkrankt. Tabelle 4 gibt eine Übersicht über die von den Studienteilnehmern aufgeführten Erkrankungen. 
Tabelle 4: Zusätzliche Erkrankungen der Studienteilnehmer

\begin{tabular}{llll}
\hline & Anzahl (n) & Prozent (\%) \\
\hline - Schilddrüsenerkrankung & 4 & 10,3 \\
- Hypertonus & 3 & 7,7 \\
- Augenerkr. (z. B. Schielen, Fehlsichtigkeit) & 3 & 7,7 \\
- Tinnitus & 2 & 5,1 \\
- Depression & 2 & 5,1 \\
- Allergien & 2 & 5,1 \\
- Endometriose & 2 & 5,1 \\
- Sonstige internistische Erkrankungen & 4 & 10,3 \\
- Sonstige orthopädische Erkrankungen & 4 & 10,3 \\
- Sonstige psychiatrische Erkrankungen & 3 & 7,7 \\
- Sonstige gynäkologische Erkrankungen & 2 & 5,1 \\
- Sonstige neurologische Erkrankungen & 2 & 5,1 \\
- Sonstige HNO-Erkrankungen & 1 & 2,6 \\
- $\quad$ Sonstige urologische Erkrankungen & 1 & 2,6 \\
\hline
\end{tabular}

\subsection{7 (Haus-) Arztsituation}

Von den 39 Studienteilnehmern gaben 92,3 \% $(n=36)$ an, einen Hausarzt zu haben. 82,1 $\%(n=32)$ hatten diesen aufgrund ihrer Kopfschmerzen bereits aufgesucht und zwar durchschnittlich 3,8 Mal. Damit nahm der Hausarzt die wichtigste Position in der Kopfschmerz-Behandlung der Teilnehmer ein. Am zweithäufigsten wurde der Neurologe aufgesucht, den 51,3\% $(n=20)$ durchschnittlich 3,3 Mal besuchten. Der Orthopäde wurde von 15 Befragten (38,5\%) im Mittel 1,6 Mal konsultiert, der Augenarzt von 14 Teilnehmern (35,9 \%) durchschnittlich 1,3 Mal. Als weiteres wurde von zwölf Betroffenen (30,8 \%) noch der HNO-Arzt in die Behandlung miteingebunden, der im Mittel 1,8 Mal konsultiert wurde. Internist, Heilpraktiker (jeweils $23,1 \%, n=9)$ und Chirotherapeut $(15,4 \%, n=$ 6) spielten eher eine untergeordnete Rolle. Dabei wurde der Internist durchschnittlich 1,5 Mal, der Heilpraktiker fünf Mal und der Chirotherapeut im Durchschnitt 2,3 Mal aufgesucht.

Auf die Frage, welche Arztbesuche in der Zukunft noch geplant seien, um die Kopfschmerzen behandeln zu lassen, antworteten elf der Befragten (28,2\%), dass sie ihren Hausarzt aufsuchen wollten. Einen Neurologen und einen Heilpraktiker wollten jeweils 
$15,4 \%(n=6)$ konsultieren. Fünf Studienteilnehmer $(12,8 \%)$ dachten darüber nach, einen Augenarzt aufgrund der Kopfschmerzen aufzusuchen, jeweils vier Betroffene (10,3\%) wollten einen Orthopäden beziehungsweise einen HNO-Arzt zu Rate ziehen. Einen Chirotherapeuten planten lediglich 7,7 \% $(n=3)$ zu besuchen, während ein Besuch des Internisten gar nicht in Erwägung gezogen wurde.

\subsubsection{Zufriedenheit mit Ärzten}

Im Hinblick auf die Zufriedenheit der Studienteilnehmer mit ihren behandelnden Ärzten ist festzustellen, dass der Hausarzt auf einer Likert-Skala von 1 - 5 (1 = überhaupt nicht zufrieden, 5 = sehr zufrieden) einen Durchschnittswert von 2,6 erreichte. Besser schnitt hierbei nur der Heilpraktiker mit einem Durchschnitt von 3,0 ab. Ebenfalls einen Wert von 2,6 erhielt der Orthopäde. Der Augenarzt erreichte einen Durchschnittswert von 2,4, dem Chirotherapeuten und dem HNO-Arzt wurde jeweils ein Wert von 2,3 zugeordnet. Am wenigsten zufrieden waren die Betroffenen mit der Behandlung ihrer Kopfschmerzen durch den Neurologen mit einem Wert von 2,2 und mit der Betreuung durch den Internisten, der mit einem mittleren Wert von 2,1 das Schlusslicht der Bewertung darstellte.

Nach der Zufriedenheit mit den Antworten des Experten befragt, vergaben die Nutzer durchschnittlich 4,1 Punkte auf der Skala.

Bei der Frage, was den Betroffenen bei ihren behandelnden Ärzten fehlen, beziehungsweise was sie stören würde, wurden unterschiedliche Aussagen gemacht. Ein wichtiger Aspekt, den insgesamt sieben der Befragten (17,9\%) in ihren Freitextantworten angaben, war die fehlende Kompetenz der behandelnden Ärzte. Ein Studienteilnehmer gab an, er habe das Gefühl, die Ärzte würden an Kopfschmerzpatienten nur „rumdoktern“ und wüssten dann „selber nicht mehr weiter“. Als mögliches „Versuchsobjekt“ sah sich ein ClusterKopfschmerz-Patient, der seinem Neurologen fehlende Fachkenntnisse bescheinigte. Ein weiterer Betroffener vermisste bei seinem Arzt die Ehrlichkeit, eventuell bestehende Wissenslücken offen zuzugeben und ihn rechtzeitig zu auf diesem speziellen Gebiet kompetenteren Fachärzten zu überweisen.

Ebenfalls ein wichtiger Kritikpunkt für die Expertenrat-Nutzer war auch das Desinteresse, was teilweise durch die in die Kopfschmerz-Therapie miteinbezogenen Ärzte zu Tage gelegt wurde. Dies bemängelten ebenfalls sieben Befragte (17,9 \%). Zum einen berichteten sie, sich bei ihrem Arzt „lästig“ zu fühlen, anderen schlug „völliges Desinteresse“ entgegen. Es wurde auch erwähnt, dass die jeweiligen Ärzte ungenügend auf die Beschwerden ihrer Patienten eingingen und teilweise keinerlei Therapieoptionen dargelegt wurden. 
Einer der Studienteilnehmer bezeichnete seinen Hausarzt gar als „Scherztherapeut“, da dieser in keiner Weise nachfragte, ob die begonnene Therapie Wirkung zeigte und sich die Beschwerden gebessert hätten.

Vier Befragte (10,3\%) sprachen deutlich aus, sich von ihrem behandelnden Arzt nicht ernst genommen zu fühlen, drei (7,7 \%) berichteten, ihr Arzt würde sich zu wenig Zeit für sie nehmen.

Ein weiterer Punkt in der Kritik der betroffenen Kopfschmerz-Patienten war außerdem die fehlende Ursachenforschung durch die Ärzte beziehungsweise die fehlende ganzheitliche Behandlung. Es würden nur Medikamente verschrieben, ohne auf mögliche Ursachen der Kopfschmerzen näher einzugehen.

Problematisch fand ein Studienteilnehmer die unterschiedlichen Meinungen der verschiedenen Fachärzte zum Symptom „Kopfschmerz“. Je nachdem, zu welchem Arzt man gehen würde, würden andere Ursachen in Betracht gezogen. Diese Tatsache würde Patienten verunsichern und sie wüssten gar nicht mehr, wem sie nun Glauben schenken sollten.

Ein Kopfschmerz-Patient bemängelte, dass die Ärzte teilweise auf einfache Lösungen nicht kommen würden und führte das Beispiel an, dass er erst in einem Forum den Tipp bekam, vor der Einnahme der Triptane Metoclopramid einzunehmen, um die Wirkung der Triptane zu verbessern. Auch auf die für ihn hilfreiche Idee, pflanzliche Pestwurzpräparate statt Betablocker oder Antidepressiva als Migräneprophylaxe auszuprobieren, habe inn erst eine Heilpraktikerin gebracht.

\subsubsection{Fühlen sich die Betroffenen mit ihren Beschwerden ernst genommen?}

Bei der Frage, ob die Befragten sich mit ihren Beschwerden von verschiedenen Personengruppen ernst genommen fühlten, wurde deutlich, dass hierbei dem familiären Umfeld die entscheidende Rolle zukam. Auf einer Skala von 1 - 5 (1 = überhaupt nicht ernst genommen, 5 = sehr ernst genommen) bekam die Familie mit 4,2 Punkten den höchsten Mittelwert zugeordnet. Dahinter folgte die Gruppe der engen Freunde. Dem behandelnden Arzt wurden im Mittel 3,3 Punkte auf der Skala zugeordnet. Die Gruppe der Bekannten bekam 3,2 Punkte, während die Teilnehmer bei den Arbeitskollegen am wenigsten das Gefühl hatten, mit ihren Kopfschmerzen ernst genommen zu werden. Die Abbildung 7 gibt eine Übersicht über die Werte im Einzelnen. Die Spannbreite der Einzelwerte der jeweiligen Personengruppen reichte dabei in allen Fällen vom Minimum 1 bis zum Maximum 5. 
Abbildung 7: Wie sehr fühlen sich die Betroffenen ernst genommen durch...

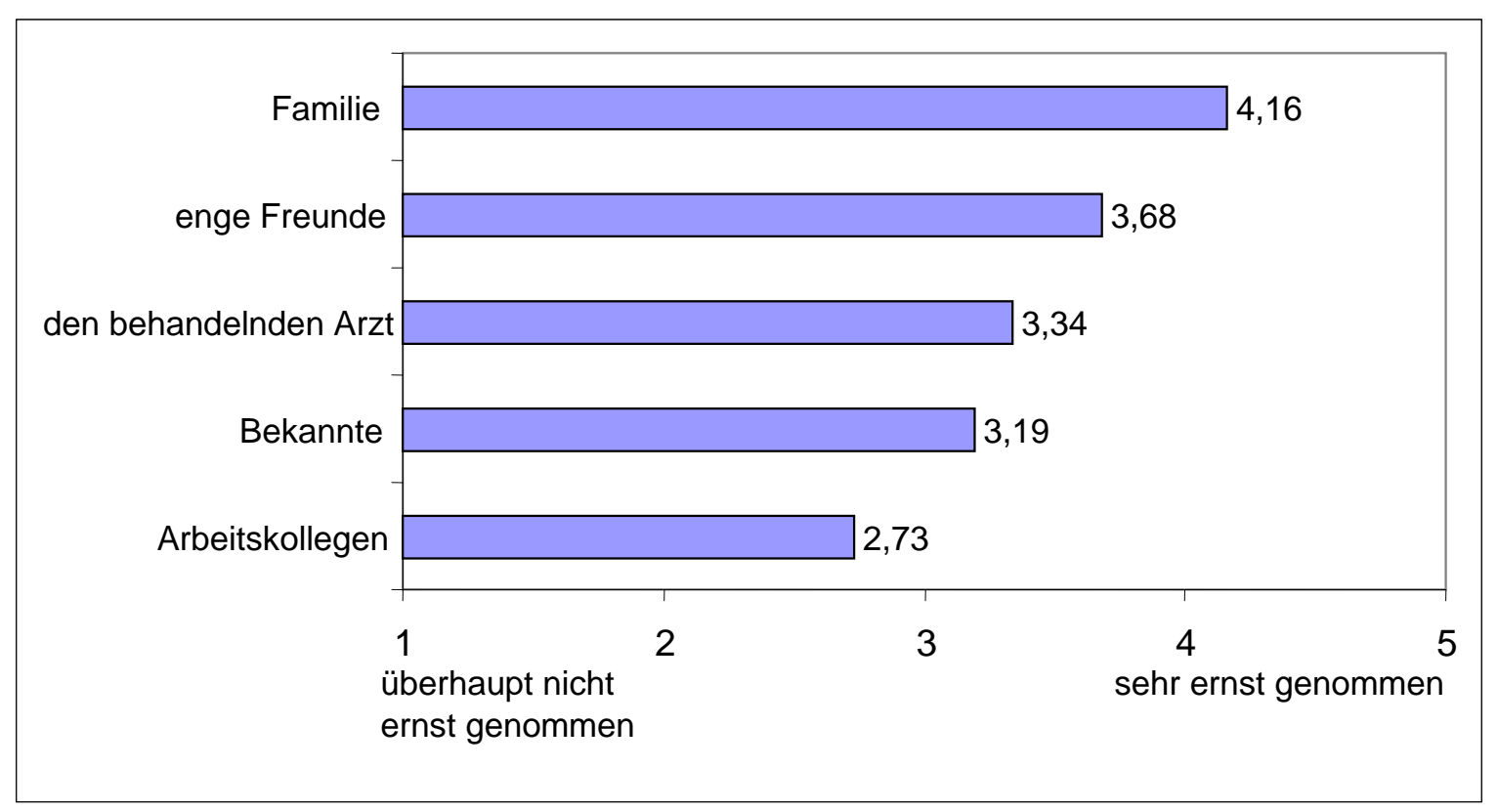

\subsubsection{Gründe für die Nutzung des Expertenrats im Internet}

Bei den Beweggründen der Benutzer, den Expertenrat zu besuchen, stand vor allem der bequeme Informationszugang im Vordergrund. Dieser wurde auf einer Skala von 1 - 5 (1 = trifft überhaupt nicht zu, 5 = trifft voll zu) mit durchschnittlich 4,4 Punkten bewertet. Von ebenfalls großer Bedeutung schienen die Zeitersparnis und der Kontakt zu anderen Betroffenen zu sein. Der Vorteil der Anonymität des Einzelnen im Internet lag mit 2,6 Punkten im Mittelfeld. Die mangelnde Kompetenz des behandelnden Arztes wurde im Durchschnitt mit 2,2 Punkten bewertet, das fehlende Vertrauen des jeweiligen Betroffenen zum eigenen Arzt lag auf der Skala bei 2,0 Punkten. Andere Gründe für die Nutzung des Expertenrates, die nicht näher erörtert wurden, wurden mit 3,0 Punkten angegeben. Die Abbildung 8 zeigt die einzelnen Werte der von den Studienteilnehmern angegebenen Gründe. Die Streuungsbreite der Werte lag auch hier zwischen dem Minimum 1 und dem Maximum 5. 
Abbildung 8: Gründe für die Nutzung des Expertenrats im Internet

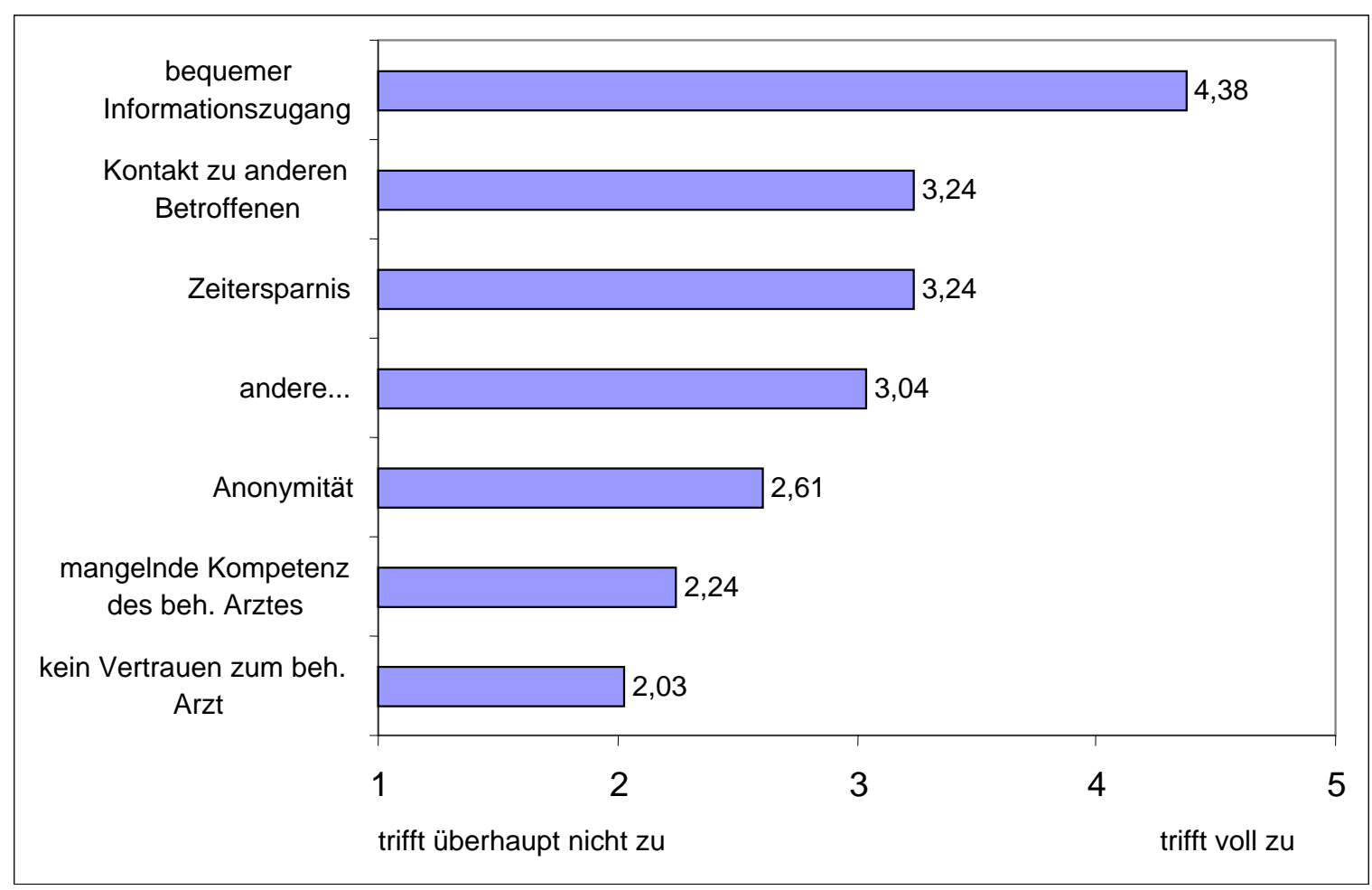

\subsubsection{Verbesserung durch den Expertenrat}

Der wichtigste Aspekt hinsichtlich einer Verbesserung der Versorgungssituation von Kopfschmerz-Patienten durch den Expertenrat war nach Meinung der Betroffenen vor allem die weiterführende Informationsgewinnung und somit ein Abbau von Unsicherheiten über die eigene Therapie. Dieser Punkt erschien in insgesamt 13 Antworten (33,3\%). Die Befragten schätzten besonders die Möglichkeit, sich in Ruhe zu Hause über mögliche Therapieoptionen zu informieren („bequem, schnell, ohne Zeitdruck und Termindruck“, „auch mit wenig Zeit und außerhalb der Praxisöffnungszeiten") und verschiedene Meinungen zu dem Thema „Kopfschmerz" miteinander zu vergleichen. Dies könne laut einigen der Studienteilnehmer „bei der Einschätzung des Arztes helfen“ und eine „Eigeneinschätzung der Symptomatik“ möglich machen. Auch sei der Expertenrat hilfreich bei der Gewinnung „eines besseren Überblicks“ und ermögliche, sich „selber ein Bild [zu] machen“. So würden zum Beispiel andere Heilmethoden aufgeführt, die der jeweilige behandelnde Arzt eventuell noch nicht aufgezeigt hatte. Die Ratschläge des Experten seien „ein bisschen ,weiterdenkend““. Ein Teilnehmer berichtete, „unzählige Tipps hinsichtlich Medikation, Erfolgsaussichten bestimmter Therapien [...] und [...] Prophylaxemöglichkeiten" bekommen zu haben. Einem anderen Studienteilnehmer war vor allem auch der Kontakt zu anderen Betroffenen wichtig. 
Der Experte und seine Ratschläge zu den individuellen gesundheitlichen Problemen wurden generell als sehr kompetent eingeschätzt. Ein Patient schrieb zum Beispiel: "Hier finde ich Ärzte, die wissen, was sie tun."

Nur zwei der Befragten (5,1 \%) beschieden dem Expertenrat „keine bis geringe“ Verbesserung der Problematik, wobei einem davon auch wiederum allein das Gefühl half, „dass man nicht alleine [sei] mit dem Problem".

\subsubsection{Fragebogen zur Differenzierung der Kopfschmerzen}

Bei der Auswertung des Kieler Kopfschmerz-Fragebogens wurde deutlich, dass der Großteil der Studienteilnehmer an Migräne litt $(59,0 \%, n=23)$. Zwei Betroffene zeigten die Symptome eines episodischen Kopfschmerzes (5,1\%), auf vier Patienten (10,3\%) trafen sowohl die Kriterien einer Migräne als auch eines episodischen Spannungskopfschmerzes zu. Insgesamt zehn Teilnehmer (25,6 \%) konnten nicht eindeutig klassifiziert werden, da sie die Kriterien der einzelnen Kopfschmerzformen nicht zur Gänze erfüllten.

Tabelle 5: Kopfschmerzarten der Studienteilnehmer

\begin{tabular}{lll}
\hline & Anzahl (n) & Prozent (\%) \\
\hline Migräne & 23 & 59,0 \\
Episodische Spannungskopfschmerzen & 2 & 5,1 \\
Migräne + episod. Spannungs-KS & 4 & 10,3 \\
Nicht klassifizierbar & 10 & 25,6 \\
\hline Gesamt & 39 & 100 \\
\hline
\end{tabular}

\subsection{Auswertung der Anfragen}

\subsubsection{Stichprobe}

In der Zeit seit Bestehen des Expertenrats in seiner zum Zeitpunkt der Erhebung aktuellen äußeren Form (April 2002) bis zur Beendigung der Online-Umfrage (Mai 2006) gingen insgesamt 894 Fragen an den Experten ein. Davon waren 59 Anfragen fehlerhaft auf der Homepage des Expertenrats gespeichert, das heißt, der Text der Fragen war gar nicht oder nur teilweise lesbar. Hierbei handelte es sich zumeist um ältere Anfragen (letzter fehlerhafter Eintrag am 24.08.2004). Somit wurden 835 Anfragen inhaltsanalytisch ausgewertet. Davon fielen 180 Mails in den Erhebungszeitraum der Online-Befragung. 


\subsubsection{Stichprobenpopulation (soziodemographische Daten)}

Auch bei den Fragestellern, die seit Frühjahr 2002 eine Anfrage gestellt hatten, war der Großteil weiblich $(65,6 \%, n=548)$. Nur $11,4 \%(n=95)$ der ausgewerteten Fragen wurden von männlichen Betroffenen gestellt. Bei 192 Anfragen (23,0 \%) konnte das Geschlecht des jeweiligen Verfassers nicht ermittelt werden, da es nicht im Text der Frage erwähnt wurde und auch nicht aus dem Namen ersichtlich war.

Das Durchschnittsalter der Nutzer des Expertenrates, die ihr Alter in ihrer Frage erwähnten, lag bei 33,2 Jahren (Minimum 14, Maximum 68, SD 11,288). 188 Frager (22,5 \%) machten diese freiwillige Angabe.

233 der Fragenden blieben vollständig anonym (27,9 \%), überwiegend wurde jedoch der Vorname angegeben $(59,3 \%, n=495) .12,8 \%(n=107)$ gaben entweder ihren vollen Namen oder nur den Nachnamen an. Die eigene E-Mail-Adresse wurde nur von 61 Nutzern veröffentlicht $(7,3 \%), 92,7 \%(n=774)$ hielten diese Information lieber zurück.

\subsubsection{Charakteristika der Anfragen}

Die Länge der Anfragen war sehr variabel. Teilweise gab es nur ganz kurze präzise Fragen, teilweise schilderten die Nutzer ihre Problematik sehr ausführlich. Die durchschnittliche Anzahl an Wörtern lag bei 107,91 Wörtern pro Frage (Minimum 5, Maximum 1075, SD 88,410).

Die meisten Fragen wurden von den Nutzern zu Themen gestellt, die sie selbst betrafen $(82,4 \%, n=688)$, nur 90 Fragesteller $(10,8 \%)$ wandten sich mit Problemen von Familie, Freunden oder Bekannten an den Experten. In einem Fall $(0,1 \%)$ wurde die Frage sowohl auf den Verfasser selbst als auch auf andere Personen bezogen. 53 (6,3 \%) der Fragen waren allgemein gefasst und bezogen sich nicht auf personengebundene Aspekte. Bei insgesamt drei Beiträgen $(0,4 \%)$ konnte die Zuordnung nicht eindeutig erfolgen.

Von den insgesamt 835 ausgewerteten Anfragen wurden 793 (95,0 \%) von dem Experten persönlich beantwortet. In 87 Fällen (10,4 \%) erfolgte auf diese Antwort eine erneute Reaktion des ursprünglichen Fragestellers, die zumeist weitergehende Fragen oder eine Danksagung für die erfolgte Antwort enthielt. Insgesamt 109 Anfragen (13,1\%) wurden neben dem Experten auch von anderen Nutzern des Expertenrates beantwortet oder kommentiert. 
Bei 42 Beiträgen (5,0 \%) handelte es sich um Fragen, in denen die Betroffenen Bezug auf früher von innen gestellte und bereits beantwortete Fragen nahmen. So wandten sich einige Nutzer erneut an den Experten, wenn z. B. neuere Untersuchungsergebnisse vorlagen oder zusätzliche Symptome auftraten. Im Hauptteil der Anfragen (95,0 \%, n = 793) wurden jedoch jeweils neue individuelle Problematiken angesprochen.

\subsubsection{Inhalte der Anfragen}

Ein Großteil der Anfragen an den Experten beschäftigte sich mit Fragen nach Symptomen $(32,2 \%, n=269)$ und speziellen Medikamenten bzw. Therapien $(32,0 \%, n=267)$. So beschrieben viele Betroffene ihre Symptome und fragten nach möglichen Diagnosen (In Klammern ist jeweils die laufende Nummer und das Datum der jeweils zitierten Anfrage angegeben. Die Zitate wurden unverändert übernommen, das heißt mögliche Rechtschreib- und Grammatikfehler wurden nicht korrigiert.):

- $\quad$ „...die Symptome waren:

Doppelbilder nur rechtsbetont

Lähmungen der rechten Zungenhälfte

Benommenheit

keinerlei Kopfschmerzen

Die Symptome sind nach ca. 6 - 7 Stunden verschwunden. [...] Können diese

Symptome tatsächlich auf eine Migräne hinweisen ?..."

(Nr. 17, 20.06.2002)

- $\quad$ „...Vor einigen Wochen hatte Sie ein besonderes Erlebnis. Sie saß mit Freunden beisammen und besprach ihre letzte (vor drei Wochen) gemeinsame Reise in ein arabisches Land. Plötzlich war Sie nicht mehr in der Lage zu sprechen. Ihrer Schilderung nach konnte Sie klar denken und auch Gedanken formulieren, das stoppte aber laut eigener Aussage beim Mund und es kam nichts sinnvolles heraus. Nach einigen Versuchen, blieb Sie ruhig und sagte nichts mehr. Nach etwa einer halben Stunden konnte Sie wieder normal reden..."

(Nr. 592, 26.02.2005)

Bei den Anfragen zu bestimmten Medikamenten und Therapien handelte es sich um Fragen, die gezielt auf Wirkungsweise, Nebenwirkungen oder Risiken etc. von einzelnen Medikamenten (-gruppen) eingingen. Auch Fragen zu bestimmten (schulmedizinischen) Therapieformen wie z. B. operative Verfahren wurden hierbei berücksichtigt. 
- $\quad$ „...welches ist das bessere Medikament bzw. der bessere Wirkstoff: Almogran (Almotriptan 12,5mg) oder AscoTop 5mg (Zolmitriptan)?

Welche entscheidenden Unterschiede gibt es in der Wirkung und Nebenwirkung? Gibt es einen großen Kostenunterschied [...] Welche Spät-Folgen hat eine "regelmäßige" (2-3x pro Monat) Einnahme von diesen Triptanen?..."

(Nr. 19, 22.06.2002)

- „ „...Wie sollte man nach 3 Jahren Annahme von Amitritilin (50 mg/Tag) dieses Madikament abzusetzen. Ich habe gelesen, dass das Medikament abhängig macht..." (Nr. 581, 17.02.2005)

Ein weiteren großen Bereich stellten die Fragen zu möglichen Therapien der Kopfschmerzen dar $(23,6 \%, n=197)$, die auf Therapievorschläge seitens des Experten abzielten:

- „..Was kann ich gegen die Migräneanfälle tun?...“

(Nr. 59, 17.09.2002)

- „ „...Was kann ich dagegen tun. Kalte Kompressen helfen nicht immer, und ich möchte nicht so starke Schmerzmittel nehmen..."

(Nr. 260, 25.06.2003)

In vielen Anfragen wurden (oftmals implizit geäußerte) psychosoziale Unterstützungsbedürfnisse deutlich $(16,9 \%, n=141)$. Viele der Betroffenen äußerten Angst, Unsicherheit und Verzweiflung im alltäglichen Umgang mit ihren Kopfschmerzen:

- $\quad$ „...ich kann noch immer nicht mit dieser ätzenden krankheit umgehen und verzweifel daran, denn die lebensqualität sinkt immer mehr!medikamente halfen nur für kurze zeit..."

(Nr. 160, 26.01.2003)

- $\quad$ „...Ich habe aber Angst vor der Untersuchung bei einem Neurologen, bei dem einen sollte ich mich total ausziehen und ich hab so doll Angst. Was könnte das sein und wie läuft eine Voruntersuchung ab ?...“

(Nr. 255, 18.06.2003)

- $\quad$ „...Weiß nicht mehr was ich machen soll.Vielleicht können Sie mir einen Rat geben. Wäre Ihnen sehr dankbar..."

(Nr. 614, 30.03.2005) 
Die Betroffenen fühlten sich allein gelassen mit ihren Beschwerden und wussten sich oftmals nicht mehr zu helfen. Die scheinbar ausweglose Situation führte häufig zu einem hohen Leidensdruck:

- $\quad$ „...Ich bekomme währenddessen totale Angstzustände so eine Art von Panik dass das nie vorbei gehen würde. [...] Das konnte ich bisher ganz gut verkraften bin aber jetzt schwanger und mache mir Sorgen wie das nach der Entbindung weitergeht. ich stoße bei meinem Mann und meiner Familie auf totales Unverständnis aber wahrscheinlich ist es auch total schwer so etwas nachzuvollziehen wenn man selbst nicht betroffen ist..."

(Nr. 312, 29.10.2003)

Ein besonders anschauliches Beispiel für diese Angst und psychische Belastung ist dieser Bericht einer Betroffenen, die sich mit ihren Sorgen an den Experten wandte:

- „Ich habe 3 Kinder und seit ca.2 Jahren angeblich Migräne.Seit November 02 ,bin ich nicht mehr ich selber.Ich lag 3 Wochen in meinem dunklem Schlafzimmer.Hatte Schwindel, Sehstörungen, Übelkeit, Lähmungen ,Hitzewallungen, Kälte, Schüttelfrost,Angstzustände, ein Ziehen über dem Auge,das über das Gesicht ging,Kribbeln und als ob ich Besoffen bin konnte überhaupt nicht am Leben teilnehmen.War bei so vielen Ärzten und nahm soviele Tabletten nichts half.Es wurde zwar ein bischen besser aber es ging nie richtig weck. Vor ca. 5 Wochen lag ich 2 Wochen im Krankenhaus, dort machten sie viele Tests, sogar wurde mir Nervenwasser entzogen, aber nichts. Vermutungen sind Migräneanfälle und Durchblutungsstörunden im Gehirn.Sie fanden im MRI Bläschen,sogenannte Narben die meist nur bei Älteren Menschen zu finden sind,aber keiner konnte mir sagen woher sie kamen.Ich nehme seitdem ich aus dem Krankenhaus draußen bin Aspirin 100, Magnesium, Gingseng, Multivitamine und ein Schildrüsenhormon das ich täglich nehmen muß. Hatte auch Vitamin b genommen aber abgesetzt.Seit Montag dem 31.3.03 geht es mir wieder schlechter, ich habe Angst.Habe Kribbeln in den Fingern,Flimmern,kann nicht richtig Denken ,Kopfdruck, Hitzewallungen,Kalte Füße und Hände,Übelkeit,bin nur halb da, wieder wie Besoffen und es kommt mir vor als will die aura der Migräne raus und kann nicht.Letzte Nacht weinte mein Baby, ich machte das licht an um zu kucken was los ist und machte dann das Licht wieder aus und da fing es an mir ganz schlecht zu werden alles wurde noch schlimmer was ich oben schon beschrieben habe und dazu kamen noch solche komischen Zuckungen, wie Schüttelfrost in der art, es ging und kam wieder das ganze für ca.20 Minuten .Ich hatte das Medikament Migränerton genommen ich weiß nicht ob das geholfen hatte aber nach einer Stunde ca. konnte ich wieder schlafen. Bitte helfen sie mir ich habe Angst.Ich treibe auch Sport, jeden tag 20 Minuten , weiß aber nicht ob ich das wei- 
ter machen soll wenn es mir so schlecht geht und weiß auch nicht mehr wann ich irgendwas einnehmen soll und was. Möchte wieder normal Leben.Ich bitte um Rat.Mit freundlichem Gruß Bianka“

(Nr. 199, 03.04.2003)

Dieses Beispiel zeigt die Beeinträchtigung von Altagstätigkeiten durch die Kopfschmerzen. Die Patientin war vermutlich nicht optimal medikamentös versorgt, obwohl sie schon einen Krankenhausaufenthalt hinter sich hatte. Der Experte empfahl ihr, sich bei einem Kopfschmerzspezialisten vorzustellen bzw. sich in eine Schmerzklinik zu begeben und bereits vorher ein Kopfschmerztagebuch zu führen, um einen eventuell medikamentös induzierten Kopfschmerz ausschließen zu können.

128 der untersuchten Anfragen (15,3 \%) beschäftigten sich mit möglichen Ursachen der Kopfschmerzen, also z. B. pathophysiologischen Vorgängen oder eventuellen Grunderkrankungen.

- $\quad$ „... Ich habe Diskusprotrusionen in C 3/4 u. C4/5, Im März sind durch meinen Orthopäden Blockaden der WS gelöst worden, seitdem habe ich extreme Schwindel, u. Migräneattacken. Könnte ein Zusammenhang bestehen?“

(Nr. 7, 06.05.2002)

- $\quad$ „...Vor einigen Tagen habe ich erfahren, dass ein Onkel von mir vor Jahren an einem geplatzten Aneurysma verstorben ist. Ich bin jetzt sehr beunruhigt. Meine Fragen:

1. Wie wahrscheinlich ist es, dass meine Symptome auch für ein Aneurysma sprechen oder könnten auch andere Ursachen in Frage kommen?..."

(Nr. 773, 05.11.2005)

Die weiteren Kategorien waren im Vergleich zu den bereits aufgeführten Themen nicht mehr ganz so häufig vertreten. So bezogen sich $10,3 \%$ der Anfragen $(n=86)$ auf weitere evtl. nötige diagnostische Schritte und spezielle, den einzelnen Nutzer betreffende Untersuchungsergebnisse. 68 Nutzer (8,1 \%) wollten vom Experten eine Empfehlung spezieller Fachärzte (teilweise auch namentlich) oder anderer Therapeuten erhalten. Der Bereich der hormonellen Ursachen bzw. der hormonellen Therapie wurde in 7,5 \% der Fragen angesprochen $(n=63)$. Die Themen „mögliche Triggerfaktoren, Auslöser oder Verstärker von Kopfschmerzen“ und „Vorbeugung und Prophylaxe“ wurden in 5,4 \% ( $n=45)$ bzw. 5,3 $\%(n=44)$ der Anfragen zur Sprache gebracht. Die weiteren Themen „alternative Therapien“ $(4,1 \%, n=34)$, „Zusammenhänge mit bestimmten Erkrankungen bzw. Folgeer- 
scheinungen“ (4,1 \%, n = 34), „Schwangerschaft und Kopfschmerz" $(2,2 \%, n=18)$ und „Einfluss von Lebensgewohnheiten“ $(2,0 \%, n=17)$ wurden seltener in den Anfragen der Nutzer angeschnitten.

58 Anfragen (6,9 \%) beschäftigten sich mit dem Thema „Kopfschmerzen bei Kindern“. Hierbei wurde häufig auch die scheinbar ausweglose Situation der Eltern deutlich, die sich oftmals nicht mehr zu helfen wussten.

- $\quad$ "Meine Tochter 7 hat seit 2,5 Jahren Migräneanfälle meistens am Wochenende oder anfangs der Woche.Das ganze nennt sie selber Augenweh denn es ist im linken Auge und zwar so heftig daß sie nicht nur einmal bricht sogar wie letzten Dienstag 6x.Sie hat schon 2 Kernspin hinter sich EEG und EKG war auch gut.Wir waren schon bei verschiedenen Heilpraktikern aber im großen und ganzen hat noch nichts geholfen.Bevor sie die Migräne bekam hatte sie zwei mal einen Unfall mit Gehirnerschütterung und kurz davor ist sie von meinen Schwiegerelterns Hund ins Gesicht gebissen worden. Sie hat in diesem Jahr als es ausgebrochen ist auch erfahren daß sie ein Geschwisterchen bekommt. Wäre froh wenn uns jemand helfen könnte denn im September soll sie in die Schule und wenn sie dort jede Woche fehlt sehe ich schwarz."

(Nr. 563, 03.02.2005)

- $\quad$ „meine 5jährige Tochter leidet seit einem Jahr ca. 2 x pro Woche an anfallsartigen Kopfschmerzen im Stirnbereich. der Schmerz tritt plötzlich auf - sie "verfällt", bekommt dunkle Ringe unter den Augen, sie ist licht- $u$. lärmempfindlich, ab und zu erbricht sie und nach max. 10 Minuten fällt Sie in einen sehr tiefen Schlaf (12-14 Stunden) aus dem man sie nicht wecken kann.

SchädeIMR: vergrößerte Liquorräume, ansonsten ob EEG: unauffällig

24 Stunden EEG: unauffällig

Diagnose: kindliche Migräne

Therapie: Tegretol (Antiepileptikum) $250 \mathrm{mg}$ retard 0/0/1

Seit Therapiebeginn haben sich die Symptome u. die Anfallshäufigkeit rapide verbessert - ich bin allerdings mit der Medikation nicht glücklich! Meine Frage: Gibt es weniger invasive Möglichkeiten um meiner Tochter zu helfen?"

(Nr. 568, 07.02.2005) 
Ein großer Teil der Anfragen bezog sich auf Themen, die nicht in eine der aufgestellten Kategorien einzuordnen waren $(13,2 \%, n=110)$ und die Aspekte enthielten, die meistens nur von einzelnen Nutzern angeschnitten wurden. Diese Fragen wurden in der Kategorie „Sonstiges“ zusammengefasst. Angesprochen wurden hierbei ganz unterschiedliche Sachen, wie z. B.:

- Chance auf Heilung der Migräne?

- $\quad$ Fragen zur genetischen Disposition, Vererbung von Kopfschmerzen

- Schwerbehinderungsanerkennung aufgrund von Migräne möglich?

- $\quad$ Finanzierung von Therapien (Heilpraktiker) über Krankenkasse?

- $\quad$ Suche nach anderen Patienten mit ähnlicher Symptomatik

- Libidoverlust durch Migräne?

- Medikament vom Markt genommen => andere Bezugsquellen (Ausland)?

- Normale“ Häufigkeit von Migräneanfällen?

- Woher Auskunft über chemische Zusammensetzung von Medikamenten?

- Was ist eine Reizverarbeitungsstörung?

- Was passiert bei Ausdauersport im Gehirn?

- Verhalten während Aura (Ruhe, Bewegung, Arbeit...)?

- $\quad$ Medikamentenentzug

- Bestellung von Medikamenten über das Internet?

- Empfehlungen von Büchern etc. zur Selbsthilfe

- $\quad$ Freund weigert sich, zum Arzt zu gehen: Was tun?

- Wie führt man ein Schmerztagebuch?

- Höheres Risiko für Höhenkrankheit beim Bergsteigen bei Migränepatienten?

In Tabelle 6 sind die verschiedenen Inhalte der Anfragen noch einmal aufgeführt. 
Tabelle 6: Inhalte der Anfragen

\begin{tabular}{lll}
\hline & Anzahl (n) & Prozent (\%) \\
\hline Symptombeschreibung & 269 & 32,2 \\
Bestimmte Medikamente/ Therapien & 267 & 32,0 \\
Mögliche Therapien & 197 & 23,6 \\
Suche nach psych. Beistand/ Hilfe & 141 & 16,9 \\
Ursachen der Kopfschmerzen & 128 & 15,3 \\
Sonstiges & 110 & 13,2 \\
Diagnostik/ Untersuchungsergebnisse & 86 & 10,3 \\
(Fach-) Arzt-/ Therapieempfehlung & 68 & 8,1 \\
Hormonelle Ursachen/ Therapie & 63 & 7,5 \\
Kopfschmerzen bei Kindern & 58 & 6,9 \\
Triggerfaktoren/ Auslöser/ Verstärker & 45 & 5,4 \\
Vorbeugung/ Prophylaxe & 44 & 5,3 \\
Alternative Therapien & 34 & 4,1 \\
Zusammenhang mit bestimmten Erkrankungen & 34 & 4,1 \\
Schwangerschaft und Kopfschmerz & 18 & 2,2 \\
Einfluss von Lebensgewohnheiten & 17 & 2,0 \\
& & \\
\hline
\end{tabular}

\subsubsection{Kritik und Kommentare}

Sehr selten wurde auch Kritik an den Antworten des Experten geäußert, zumeist von anderen Nutzern, die mit dem Inhalt der Antwort nicht einverstanden waren und eine andere Meinung vertraten. Beispielsweise gab es einmal einen Beitrag eines Nutzers, der mit einer Empfehlung des Experten zu Triptanen nicht einverstanden war:

- „Unmöglicher Empfehlung!!!!

ich hatte mich zuerst gewundert, dass ein Expertenteam Naratriptan so in den Vordergrund stellt, aber nachdem ich gesehen habe von wem diese Seite gesponsert wird war mir einiges klar!(Schwarz Pharma=stellt Naratriptan her) Naratriptan hat zwar eine lange Wirkdauer, jedoch einen sehr langen Wirkeintrit von bis zu 3 Stunden, was bei einer starken Migräne nicht sehr hilfreich ist. Desweiteren wurde beispielsweise Almotriptan mit einer $24 \mathrm{~h}$ Wirkung und einem Wirkeintritt von 30 min als weiterer Wirkstoff total vergessen! Die Parameter für ein Triptan sollten der schnelle Wirkeintritt, die Schmerzfreiheit nach 2 h und die Wiederkehrkopfschmerzrate sein.

Es ist daher meiner Meinung nach äußerst unprofessionell Naratriptan so in den 
Vordergrund zu stellen, da es bis auf einen langen Wirkeintritt nichts hat, was ein gutes Triptan ausmacht!

Siehe auch Metaanalyse von Ferrari erschienen im Lancet!!"

(Nr. 420, 23.03.2004)

Der Experte bezog auf diese Vorwürfe in seiner Antwort Stellung und verteidigte seine Empfehlungen:

- $\quad$ „es ist nicht zutreffend, dass ich als Experte ein Triptan stärker in den Vordergrund stelle. Meine Beratungstätigkeit übe ich vollständig unabhängig aus. Gegenüber Pharmafirmen bestehen keinerlei Verpflichtungen.

Die Bewertung der Triptane erfolgt, wie Sie richtig schreiben, nach der Schnelligkeit des Wirkeintritts, der Schmerzfreiheit nach 2 Stunden und nach der Wiederkehrkopfschmerzrate.

Der Wirkeintritt erfolgt am schnellsten bei Sumatriptan subcutan. Bereits nach ca. 10 Minuten kann eine klinische Wirksamkeit erreicht werden. Auch Sumatriptan nasal hat mit ca. 15 Minuten einen schnellen Wirkungseintritt. Wird Sumatriptan als Tablette verabreicht, beträgt die Dauer bis zum Wirkeintritt 30 Minuten. Auch Rizatriptan, Eletriptan und Almotriptan benötigen 30 Minuten. Bei Zolmitriptan sind es 45 Minuten, bei Naratriptan 60 Minuten.

Kopfschmerzfreiheit nach 2 Stunden läßt sich bei vergleichbarer Dosierung durch Sumatriptan, Zolmitriptan, Rizatriptan, Almotriptan und Eletriptan in gleicher Weise bei etwa $20 \%$ der Patienten erreichen. Bei Naratriptan sind es etwa $12 \%$.

Die Wiederkehrkopfschmerzrate beträgt bei Rizatriptan $40 \%$, bei Sumatriptan und Zolmitriptan $32 \%$, bei Eletriptan $24 \%$, bei Almotriptan $23 \%$, bei Naratriptan $20 \%$. Die Nebenwirkungen der Triptane sind von der Dosierung abhängig. Der durchschnittliche Prozentsatz der Patienten, die nach Abzug der Placeborate über Nebenwirkungen klagten, liegt bei Sumatriptan 50 mg bei $8 \%$, bei Sumatriptan 100 mg bei $12 \%$, bei Zolmitriptan 2,5 mg bei $16 \%$, bei Zolmitriptan $5 \mathrm{mg} 24 \%$, Rizatriptan $5 \mathrm{mg}$ bei $8 \%$, Rizatriptan $10 \mathrm{mg}$ bei $14 \%$, Eletriptan $40 \mathrm{mg}$ bei $8 \%$, Eletriptan $80 \mathrm{mg}$ bei $18 \%$,Almotriptan bei $3 \%$, Naratriptan bei $3 \%$.

Mit freundlichem Gruß

Dr. Mildenstein“

Ein weiterer Nutzer, der anonym blieb, kommentierte die Antworten des Experten bei mehreren Anfragen und vertrat vehement eine andere Meinung. Der Experte bezog auch hier stets selbst Stellung zu diesen Anmerkungen. Die Beiträge wurden im Nachhinein von der Redaktion „Lifeline“ gelöscht, vermutlich um die Verbreitung von falschen Informationen zu verhindern. 
Generell fand jedoch wenig Diskussion im Bereich des Expertenrats statt. Zwar gab es bei 13,1 \% der Fragen Reaktionen von anderen Nutzern, in denen den Betroffenen teilweise auch Tipps zur Lösung des Problems gegeben wurden, vielfach erfolgte aber auch nur eine Schilderung der eigenen, oft ähnlichen Problematik, an die sich oftmals eine eigenständige Frage an den Experten anschloss. 


\section{Diskussion}

In dieser Arbeit wurden die an der Studie teilnehmenden Kopfschmerzpatienten nach ihrer Motivation befragt, den Kopfschmerz-Expertenrat im Internet aufzusuchen. Hierbei wurden neben der Auswertung eines Internet-Fragebogens auch die Anfragen an den für den Expertenrat zuständigen Allgemeinarzt analysiert. Auf diese Weise sollten die Beweggründe der Betroffenen für die Nutzung des Expertenrates analysiert werden.

Es zeigte sich, dass die Mehrzahl der Nutzer den Expertenrat aufgrund seiner Möglichkeit der schnellen, unkomplizierten und bequemen Informationsgewinnung schätzt. Bei der Inhaltsanalyse der Anfragen wurde jedoch auch deutlich, dass sich viele der Nutzer in letzter Instanz an den Expertenrat auf der Suche nach Hilfe wandten. Generell kann das Internet keinen Ersatz für den persönlichen Arzt-Patienten-Kontakt darstellen, es kann aber sehr wohl als Ergänzung der konventionellen medizinischen Betreuung dienen.

\subsection{Diskussion der Methode}

Es wurden in dieser Arbeit zwei Methoden miteinander kombiniert, zum einen wurde ein Internet-Fragebogen verwendet, zum anderen wurden die Anfragen der Nutzer an den Expertenrat inhaltsanalytisch ausgewertet. Diese Methode des cross sectional survey erlaubte einen Querschnitt durch die aktuelle Population der Expertenrat-Nutzer. Durch die Kombination von Fragebogen und Inhaltsanalyse wurde ermöglicht, die Studienteilnehmer und Nutzer des Expertenrats besser und näher zu charakterisieren, da die Inhalte der Anfragen ebenfalls Aufschluss über die Motivation der Nutzer verrieten, sich mit ihren Problemen an den Kopfschmerz-Experten zu wenden.

\subsubsection{Fragebogenerhebung}

Der selbst erstellte Fragebogen wurde hinsichtlich seiner Validität und Verständlichkeit in der Abteilung für Allgemeinmedizin der Universität Göttingen in mehreren Feedbackschleifen entwickelt. Er war einfach strukturiert und die Fragen wiesen eindeutige Formulierungen auf, so dass bei der Bearbeitung des Fragebogens durch die Expertenratnutzer keine Verständnisschwierigkeiten auftreten konnten. Der Kieler Kopfschmerz-Fragebogen von Herrn Prof. Hartmut Göbel ermöglicht eine einfache und unkomplizierte Informationsgewinnung über die Ausprägung von Migräne und Spannungskopfschmerzen mittels einer standardisierten, reliablen und validen Methode (Göbel 1994). Er wurde auf seine Validität und Reliabilität hin in einer klinischen Studie untersucht und auch im klinischen Alltag erprobt. Die beiden Fragebögen schlossen nahtlos aneinander an, so dass für die Studien- 
teilnehmer ein einheitliches optisches Bild entstand. Die Auswertung des Kieler Kopfschmerz-Fragebogens erfolgte streng nach dem vorgegebenen Auswertungsschema, um die Validität der Daten zu gewährleisten.

\subsubsection{Inhaltsanalytische Auswertung der Anfragen}

Die Anfragen, die in dieser Studie untersucht wurden, bilden die Gesamtanzahl der seit der Einführung des Expertenrates „Kopfschmerz“ auf der Lifeline-Homepage bis zum Ende des Erhebungszeitraums gestellten Fragen ab. Die Fragen wurden neben der Erhebung von statistischen Angaben in unterschiedliche Kategorien eingeteilt, die sich auf den Inhalt der Anfrage bezogen. Hierbei war die Einteilung einer Anfrage in mehrere Kategorien zulässig. So konnten die Anfragen inhaltlich analysiert und die in ihnen behandelten Themen dokumentiert werden. Hierbei wurde nach dem Prinzip der Inhaltsanalyse vorgegangen, wie es durch Krippendorff beschrieben wurde (1980) und welches drei Punkte umfasst: Das Erstellen und die Durchführung der Studie sowie die Dokumentation der Ergebnisse. Diese Art der Inhaltsanalyse ermöglicht das Umgehen mit unstrukturiertem Material (wie in diesem Fall Freitext-Anfragen) und das Erfassen von größeren Datenmengen (Krippendorff 1980). Der Vorteil ist, dass es sich hierbei um eine nicht-reaktive Technik handelt, die keinen Einfluss auf die Studienergebnisse nimmt und kontextsensitiv anzuwenden ist.

\subsubsection{Limitationen der Fragebogenerhebung}

Mit insgesamt 61 ausgefüllten und abgeschickten Fragebögen ergibt sich eine Rücklaufquote von 33,9 \%. Da 22 ausgefüllte Fragebögen die Einschlusskriterien nicht erfüllten und daher nicht in die Auswertung aufgenommen wurden, nahmen somit 21,7 \% der Expertenratnutzer, die im Erhebungszeitraum eine eigene Anfrage an den Experten gestellt hatten, an der Studie teil. Die geringe Rücklaufquote lässt sich durch vier unterschiedliche Faktoren erklären.

1. Strenge Einschlusskriterien

2. Niedrigere Rücklaufquote bei Internetumfragen allgemein

3. Auflagen der Ethikkommission

4. Technische Schwierigkeiten

Zu 1.: Die Einschlusskriterien umfassten, dass die Nutzer eine eigene Anfrage an den Experten gestellt und in den letzten sechs Monaten Kopfschmerzen gehabt hatten. Diese recht strengen Kriterien führten dazu, dass 22 der 61 ausgefüllten Fragebögen in der Auswertung nicht berücksichtigt wurden. 
Zu 2.: Das bekannte Problem der schlechten Rücklaufquoten bei Umfragen ist wahrscheinlich zu einem großen Teil durch die Übersättigung und Antwortmüdigkeit der angesprochenen Personen zu erklären. Dies betrifft insbesondere auch internetbasierte Befragungen (Wyatt 2000). In verschiedenen Studien hat sich gezeigt, dass die Rücklaufquoten internetbasierter Umfragen zumeist niedriger sind als von konventionellen, also papierbasierten Befragungen (Mavis und Brocato 1998, Leece et al. 2004). So wurden in einer Studie von Van Den Kerkhof et al. knapp halb so viele Fragebögen per E-Mail beantwortet wie per Brief (35 \% vs. 69 \%) (2004). Dahingegen stellten Truell et al. fest, dass bei beiden Umfrageverfahren (E-Mail vs. postalisch) eine ähnliche Rücklaufquote zu verzeichnen war (2002). Zudem seien die Antworten der Befragten per E-Mail deutlich schneller eingetroffen als jene auf postalischem Weg und die per E-Mail verschickten Umfragebögen seien eher vollständig ausgefüllt worden. Mit dem zunehmenden Einzug des Internets in das alltägliche Leben scheint sich die Zuverlässigkeit und Aussagekraft von internetbasierten Umfragen zu steigern, wie eine neuere Studie von Kongsved et al. belegt (2007). Nach einmaliger Erinnerung der Studienteilnehmer wurden 64,2 \% der internetbasierten und 76,5 \% der Papier-Fragebögen zurückgeschickt. Erwähnenswert ist hier vor allem, dass $97,8 \%$ der Internetfragebögen komplett, das heißt ohne fehlende Daten ausgefüllt wurden. Bei der Papierform war dies nur bei 63,4 \% der zurückgeschickten Fragebögen der Fall. Allerdings sind internetbasierte Umfragen zu wissenschaftlichen Zwecken nach Meinung der Autoren noch nicht dazu geeignet, in großem Umfang eingesetzt zu werden. Vielmehr seien sie zurzeit nur bei ausgesuchten Populationen empfehlenswert, die den Entwicklungen des Internets offen gegenüber stehen. Die Nutzer des KopfschmerzExpertenrats gehören dieser Gruppe sicherlich an, da sie das Internet bereits in ihr tägliches Leben integriert haben, wie die Auswertung des allgemeinen Internetverhaltens der Nutzer zeigt. Somit entsprechen sie den Kriterien für die Durchführung einer internetgestützten Befragung (Wyatt 2000). Zudem war es nur mittels des Internets möglich, Kontakt mit den Expertenratnutzern aufzunehmen, da in den Anfragen zumeist keine Kontaktangaben gemacht wurden.

Zu 3.: Eine wichtige Rolle spielte sicherlich die Präsentation der Umfrage und des Fragebogens. Aufgrund der datenschutzbezogenen Auflagen der Ethikkommission musste dem eigentlichen Fragebogen ein Text vorgeschaltet werden, in dem die Studie kurz vorgestellt wurde und eine Aufklärung über die Datensicherheit und Gewährung der Anonymität des einzelnen Nutzers erfolgte. Erst durch Herunterscrollen der Seite gelangte man zu dem unter dem Text stehenden Link, der den Nutzer letztendlich zum Fragebogen führte. Dies führte sicherlich dazu, dass einige Nutzer die Seite sofort wieder schlossen, ohne sich den Text durchzulesen oder den Fragebogen aufzurufen. Außerdem wandten sich einige 
Betroffene mit akuten Beschwerden an den Experten, die sich vermutlich nicht in der Lage sahen, den Text zu lesen oder den Fragebogen zu bearbeiten.

Zu 4.: Es ergab sich ein weiteres technisches Problem, das sich leider trotz aller Bemühungen nicht beheben ließ. Nach Klicken auf den Link zum Fragebogen öffnete sich ein Hinweisfenster, das auf ein abgelaufenes Sicherheitszertifikat der Internetseite hinwies, auf der sich der Fragebogen befand. Nur durch Beantworten der Frage „Soll der Vorgang fortgesetzt werden?" mit "ja“ durch Klicken auf den entsprechenden Button öffnete sich dann der Fragebogen. Wurde auf "nein“ geklickt, so öffnete sich die Seite nicht. Dieses Problem lag an einem abgelaufenen Sicherheitszertifikat der verwendeten Datenbank, welches durch die Informatiker der Abteilung für Allgemeinmedizin der Universität Göttingen aufgrund von fehlenden Administratorrechten am entsprechenden Programm nicht geändert werden konnte.

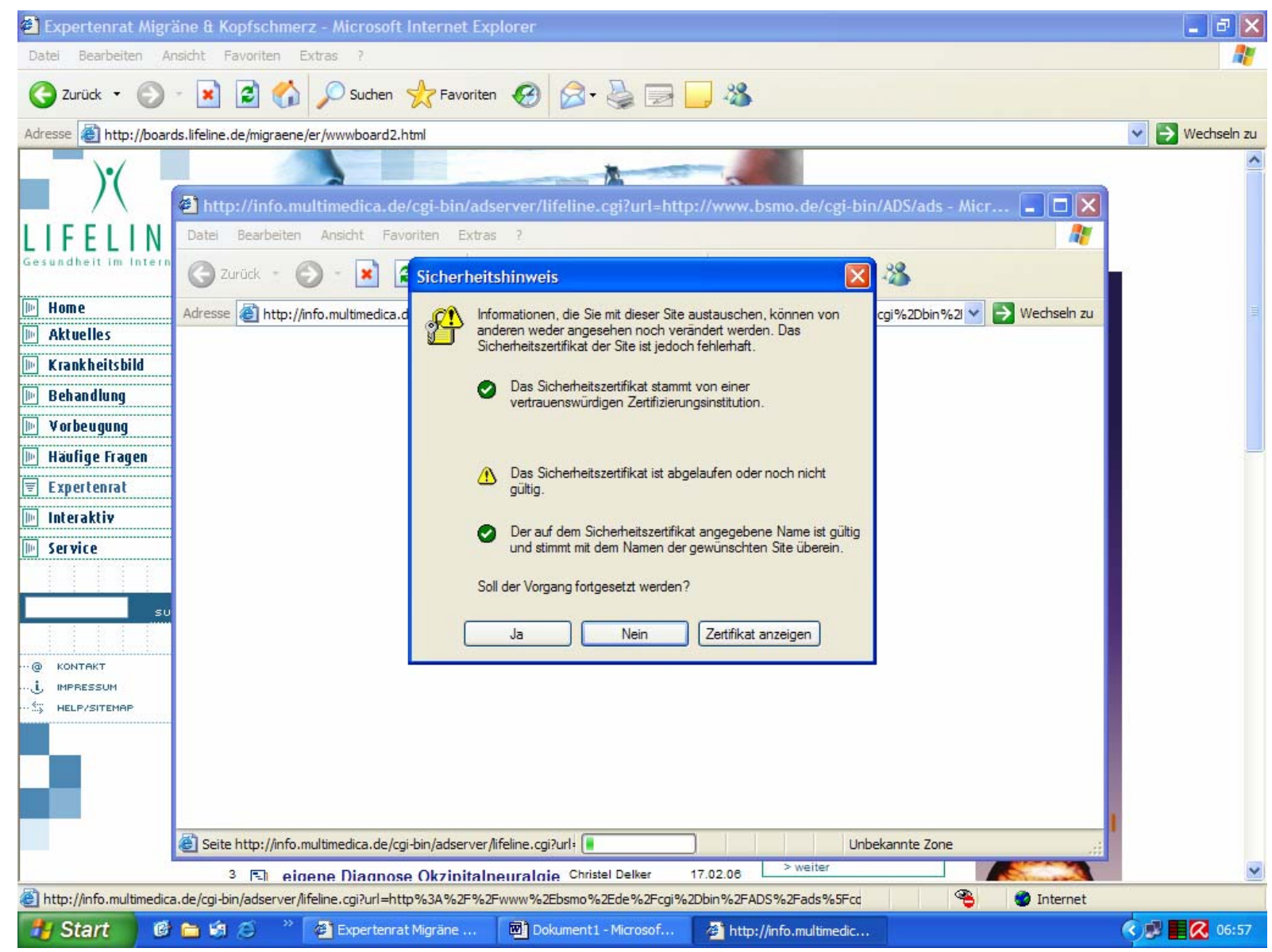

Abbildung 9: Sicherheitshinweis über abgelaufenes Sicherheitszertifikat 
Eine Besucherin des Expertenrats meldete sich per E-Mail, da sie die Teaserbox mit dem Link zum Fragebogen nicht finden konnte. Daraufhin wurde, wie bereits oben beschrieben, die Lokalisation dieser Teaserbox in den Aufforderungstexten noch näher beschrieben.

Trotz der geringen Rücklaufquote war die Durchführung der Online-Befragung der Expertenrat-Nutzer erfolgreich, da sie Aufschluss über die individuelle Vorgeschichte und die Erfahrung der Ratsuchenden mit behandelnden Ärzten gab. Die Nutzer des Expertenrates konnten somit besser charakterisiert und ihre Anforderungen an die ärztliche Betreuung eruiert werden. Zudem konnte die Zufriedenheit mit den Antworten des Experten evaluiert werden.

\subsubsection{Limitationen der inhaltsanalytischen Auswertung}

Auch die Inhaltsanalyse der an den Expertenrat gestellten Fragen ist in einigen Punkten in ihrer Aussagekraft begrenzt. So erfolgte eine annähernde Einkategorisierung der einzelnen Anfragen, das heißt die Inhalte der Anfragen wurden denjenigen vorher festgelegten Kategorien zugeordnet, mit denen sie am besten übereinstimmten. Außerdem lagen kaum Zusatzinformationen zu den einzelnen Nutzern vor, nur wenige machten die freiwillige Angabe über ihr Alter oder eventuelle Komorbiditäten. Auch das Geschlecht wurde nicht von allen Fragestellern preisgegeben. Die Angaben über die Krankheitsgeschichte oder den eigenen Leidensdruck der einzelnen Nutzer in ihren jeweiligen Anfragen waren subjektiv und somit nicht einheitlich bewertbar. Insgesamt gesehen sind die Ergebnisse daher nicht gut generalisierbar und nur bedingt auf andere Populationen übertragbar.

\subsection{Diskussion der Ergebnisse}

\subsubsection{Studienpopulation}

Die Studienpopulation bestand aus zwei Gruppen, zum einem aus dem Fragebogenkollektiv, zum anderen aus dem Anfragenkollektiv. Das Fragebogenkollektiv umfasste jene 39 Nutzer des Expertenrates, die den Fragebogen vollständig ausgefüllt hatten und die Einschlusskriterien erfüllten. Im Anfragenkollektiv wurden alle Nutzer zusammengefasst, die im Zeitraum von April 2002 bis Mai 2006 insgesamt 835 auswertbare Anfragen an den Expertenrat gestellt hatten. Die soziodemographischen Daten dieser Gruppe sind nur eingeschränkt auszuwerten, da die Nutzer nicht explizit hiernach gefragt und diese freiwilligen Angaben nicht immer gemacht wurden. Zudem kann nicht ausgeschlossen werden, dass ein und derselbe Fragesteller mehrmals eine Frage an den Experten richtete, da dies nicht immer klar erkennbar war, und somit mehrfach in der Auswertung berücksichtigt 
wurde. Aufgrund der geringen Rücklaufquote (vgl. Kapitel 6.1.3) können die Daten der relativ kleinen Stichprobe des Fragebogenkollektivs ebenfalls nur beschränkt bewertet werden.

Der Internetfragebogen wurde größtenteils von Frauen beantwortet, nur 10,3 \% der abgeschickten Fragebögen stammten von Männern. Ein ähnlicher Prozentsatz ergab sich bei der Auswertung der Anfragen, nur 11,4 \% der an den Experten gestellten Fragen stammten von männlichen Nutzern. Allerdings lässt sich dieser Prozentsatz nur bedingt bewerten, da sich bei immerhin $23 \%$ der Anfragen das Geschlecht des jeweiligen Fragestellers nicht ermitteln ließ. Dies steht im Einklang zu den Tatsachen, dass zumeist Frauen von Kopfschmerzen betroffen sind (Rasmussen et al. 1991, Lyngberg et al. 2005 a, Lipton et al. 2001) und auch vor allem junge Frauen eher einen Arzt aufgrund ihrer Kopfschmerzsymptomatik aufsuchen (Thomas et al. 2004). Umefjord et al. untersuchten eine schwedisches Expertenratforum im Internet, bei dem der typische Nutzer weiblich und zwischen 21 und 60 Jahren alt war (2008). Drei Viertel der Anfragen wurden hier von weiblichen Nutzern gestellt.

Das Durchschnittsalter der beiden Gruppen war mit 33,8 (Fragebogenkollektiv) bzw. 33,2 (Anfragenkollektiv) Jahren eher niedrig. Dieses lässt sich einerseits mit dem Altersgipfel von Kopfschmerzen in den jüngeren bis mittleren Altersstufen erklären (Rasmussen et al. 1991, Lipton et al. 2002), andererseits spielt hierbei sicherlich auch das eher niedrigere Durchschnittsalter der Internetnutzer eine Rolle (Pennekamp et al. 2006, Lenert et al. 2002). Vor allem gehören gerade Patienten, die Internetsprechstunden nutzen, eher der jüngeren Generation an (Ramos et al. 2004). Arican führte eine Studie durch, in der EMails von Patienten untersucht wurden, die an einen Expertenrat für Dermatologie gestellt wurden (2007). Das Durchschnittsalter der Nutzer, die in ihrer Anfrage ihr Alter angaben (34,3\% der Anfragen), betrug hier 29,3 Jahre.

$74,4 \%$ der an der Internetbefragung teilnehmenden Nutzer litten der Auswertung des Kieler Fragebogens gemäß an Migräne, episodischen Spannungskopfschmerzen oder beidem, wobei die Migräne den Großteil der Erkrankungen ausmachte. Bei 25,6 \% konnten die Kopfschmerzen nicht näher klassifiziert werden, da sie keiner der durch den Kieler Kopfschmerzfragebogen evaluierbaren Kopfschmerzarten eindeutig zugeordnet werden konnten. Die befragten Expertenratnutzer litten also tatsächlich an therapiebedürftigen Kopfschmerzen und stellten somit ernst zu nehmende Patienten dar. Es ist also zu vermuten, dass der Großteil der Betroffenen, die innerhalb des Expertenrates eine Anfrage stellen, ernsthaft an Kopfschmerzen erkrankt ist und durchaus einer ärztlichen Therapie und Betreuung bedarf. 
Bei der Frage, inwieweit die Aussagen der Betroffenen der Wahrheit entsprechen, lässt sich eine Studie von Lenert et al. anführen, die zeigte, dass $97 \%$ der Patienten, die im Internet angaben, an Migräne zu leiden, auch tatsächlich von Migräne betroffen waren (2002). Die Patientenangaben stellen also keinen Unsicherheitsfaktor hinsichtlich valider Untersuchungen dar.

\subsubsection{Leitliniengerechte Therapie der Nutzer}

Interessant ist die Tatsache, dass laut Auswertung des Kieler Fragebogens insgesamt 23 Studienteilnehmer an Migräne litten (teilweise kombiniert mit episodischen Spannungskopfschmerzen), jedoch nur 16 dieser Patienten angaben, Triptane zu nehmen. Dabei stellen laut den Leitlinien der Deutschen Gesellschaft für Neurologie die Triptane (5HT1B/ 1D-Agonisten) die wichtigste Substanzgruppe in der Behandlung des akuten Migräneanfalls dar, da sie die beste Wirksamkeit und eine gute Verträglichkeit haben (Kommission „Leitlinien der Deutschen Gesellschaft für Neurologie“ 2008 a). Häufig werden jedoch die betroffenen Patienten mit älteren Medikamenten behandelt, die vermutlich weniger effektiv sind (Gibbs et al. 2003), oder unzureichend medikamentös eingestellt (Gupta et al. 2007). Auch die Möglichkeiten der medikamentösen Migräneprophylaxe werden vielfach nicht voll ausgeschöpft (Stark et al. 2007).

\subsubsection{Der chronische (Kopf-) Schmerzpatient - ein schwieriger Patient?}

Die Mehrzahl der befragten Nutzer des Expertenrates gab an, neben den Kopfschmerzen noch andere Erkrankungen zu haben, hauptsächlich hormoneller Ursache (Schilddrüsenerkrankungen). Fünf Patienten gaben psychiatrische Erkrankungen an (Angststörung, Panikattacken, Depression, Borderline-Störung). In der Literatur finden sich verschiedene Arbeiten, die zeigen, dass Kopfschmerzpatienten häufig anfällig sind für psychiatrische Erkrankungen. So besteht eine Assoziation zwischen Migräne und schweren Depressionen oder Angststörungen, weswegen Migränepatienten sorgfältig auf Depressionen hin untersucht werden sollten (Frediani und Villani 2007). Von Migräne betroffene Patienten sind zudem drei bis zehn Mal so stark gefährdet, an Angststörungen und Panikattacken zu leiden (Baskin 2007). Sie zeigen häufig depressive Symptome und eine veränderte Aggressionsbewältigung mit einem Hang zu Kontrollzwängen. Daher sind sie empfindsamer für Stress und können schlechter mit ihren Schmerzen umgehen (Abbate-Daga et al. 2007). Auch die ständige sorgenvolle Erwartung des nächsten Migräneanfalls kann negativen Einfluss auf das alltägliche Leben der Betroffenen haben (Freitag 2007). Insgesamt sind Patienten mit chronischen Kopfschmerzen eher von diesen Problemen betroffen als Patienten mit episodischen Kopfschmerzen (Wiendels et al. 2006 a, b, Baskin 2007). 
Diese Tatsachen legen nahe, dass Kopfschmerzpatienten das Gesundheitswesen und ihre behandelnden Ärzte stark beanspruchen. Tatsächlich nehmen Patienten, die aufgrund ihrer chronischen Schmerzen eingeschränkt sind, häufiger als andere Gesundheitsleistungen (den Besuch des Hausarztes eingeschlossen) in Anspruch (Blyth et al. 2004, Vinding et al. 2007). Über die Hälfte der Schmerzpatienten einer Studie waren regelmäßige Nutzer des Gesundheitswesens im Vergleich zu einem Drittel der Kontrollgruppe (von Korff et al. 2007). Die Häufigkeit der Arztbesuche hängt hierbei von der Art des Kopfschmerzes ab, Migränepatienten suchen ihren Hausarzt am häufigsten auf (Hasse et al. 2002). Diese Patienten suchen allerdings nicht nur nach einer effektiven Schmerztherapie, sie suchen auch nach dem Grund ihrer Erkrankung und benötigen die Versicherung, dass ihren Kopfschmerzen keine maligne oder ernst zu nehmende Erkrankung zu Grunde liegt. Für viele Kopfschmerzpatienten ist letzteres ein wichtiger Aspekt (Leiper et al. 2006). Regelmäßige Folgekonsultationen seien nötig, um den Patienten das Gefühl geben zu können, sie in die Kopfschmerztherapie mit einzubeziehen (MacGregor 1997). Auch die in unserer Studie befragten Expertenratnutzer beklagten teilweise die fehlende Ursachenforschung durch ihre behandelnden Ärzte sowie die mangelnde Darlegung verschiedener Therapieoptionen.

In der hausärztlichen Praxis werden die anspruchsvollen chronischen Kopfschmerzpatienten häufig als schwierig zu führen angesehen. Vor allem fordernde Patienten mit häufig therapieresistenten chronischen Schmerzen und medizinischen Problemen wie multiplen, anhaltenden Schmerzen, dem Wunsch nach ausreichender analgetischer Medikation oder psychiatrischen Komponenten erschweren die unvorbelastete Begegnung in der hausärztlichen Umgebung (Elder et al. 2006). Wilm et al. zeigten in einer Studie, dass bei gleicher Anamnese und gleichen Beschwerden bei ängstlich wirkenden Kopfschmerzpatienten mehr kostenträchtige und weiterführende Untersuchungen und Überweisungen veranlasst wurden, die medizinisch eigentlich nicht indiziert waren (2008).

\subsubsection{Patientenzufriedenheit mit der ärztlichen Versorgung}

Die befragten Nutzer des in unserer Studie betrachteten Expertenrats gaben bei der Frage nach der Zufriedenheit ihrer Kopfschmerzbehandlung durch ihren Hausarzt eine eher durchschnittliche Zufriedenheit an. Trotzdem wurde die Behandlung durch den Hausarzt von allen ärztlichen Behandlungen noch am besten beurteilt. Kritikpunkte lagen vor allem bei der fehlenden Kompetenz der Ärzte in Bezug auf eine adäquate Kopfschmerztherapie und bei der fehlenden Empathie des behandelnden Arztes. Jeweils 17,9 \% der Befragten bemängelten diese beiden Aspekte. Einige fühlten sich „lästig“ oder beschieden dem Arzt Desinteresse für die jeweilige Symptomatik der Patienten. In Studien zeigte sich, dass nur 
ein kleiner Teil der Migränepatienten (34 \% bzw. 29 \%) vollends zufrieden ist mit ihrer individuellen Therapie (Lake 1999, Lipton und Stewart 1999). Der Großteil ist einigermaßen zufrieden, wobei Hauptkritikpunkt an der medikamentösen Therapie ist, dass der Wirkeintritt zu lange dauert (Lipton und Stewart 1999). Das Erreichen der Schmerzfreiheit ist das wichtigste Kriterium für eine schnell zu erzielende Zufriedenheit der Betroffenen (Davies et al. 2000). In einer neueren Studie zeigten Walling et al. eine Zufriedenheit behandelter Patienten von 74 \%, wovon 18 \% sogar sehr zufrieden mit ihrer Migränetherapie waren (2005). Trotzdem berichtet über ein Drittel der Patienten einer norwegischen Studie, dass ihre Ärzte nicht den Einfluss ihrer schweren Kopfschmerzen auf das alltägliche Leben verstehen würden (Dueland et al. 2005).

Die Zufriedenheit der Kopfschmerzpatienten hängt also von verschiedenen Faktoren ab: Neben dem möglichst schnellen Handeln des Arztes, Erreichen der Schmerzfreiheit, dem Nicht-Wiederauftreten der Schmerzen und dem Fehlen von Nebenwirkungen (LanteriMinet 2005) steht das Verständnis und die Empathie des behandelnden Arztes im Vordergrund. Wichtig ist aber auch, wie viel Zeit dem einzelnen Patienten zuteil wird, dies spielt eine bedeutende Rolle in der Kopfschmerztherapie (Manzoni und Torelli 2007). Verschiedene Faktoren stehen dem Gelingen einer für beide Seiten (Arzt und Patient) zufrieden stellenden Therapie im Weg: Nicht nur fehlende Kompetenz des Arztes und organisatorische Absurditäten (Manzoni und Torelli 2007), sondern auch die unzureichende Diagnosestellung und medikamentöse Therapie (Lipton et al. 2003 a) beeinflussen das Ergebnis negativ. Lipton et al. fassten diese Probleme in drei Stufen zusammen (1994): Zunächst gehen viele Migränepatienten nicht zum Arzt, dann wird häufig eine falsche Diagnose gestellt und zuletzt eine ineffektive Therapie verordnet. Mögliche Gründe für den nicht erfolgten Arztbesuch bei Kopfschmerzen analysierten Leiper et al. (2006). Neben der vermuteten Zeitknappheit des Arztes wurden hier vorangehende schlechte Erfahrungen mit Ärzten angegeben. Außerdem stellten die Fragen nach den Kosten der Behandlung und die Angst, was der Arzt wohl über den einzelnen Kopfschmerzpatienten denken würde, Hindernisgründe für die Patienten dar.

\subsubsection{Patientenwünsche an ihre medizinische Versorgung}

Die Hauptwünsche der Kopfschmerzpatienten, ihre individuelle Therapie betreffend, lassen sich in einigen Punkten zusammenfassen: Neben der Einbeziehung von alternativen Therapien ist vor allem die während eines Arztbesuches zur Verfügung stehende Zeit entscheidend. Auch die Bereitschaft des Arztes, sich über Symptome und (Kopfschmerz-) Erkrankungen genügend zu informieren, ist in den Augen der Patienten wichtig (Cottrell et al. 2001). Zudem möchten die Patienten aktiv in die Entscheidung über Therapien einbe- 
zogen werden und über mögliche Nebenwirkungen hinreichend aufgeklärt werden (Rozen 2006). Dieser Wunsch wurde auch in den Äußerungen der Expertenrat-Nutzer deutlich. Die Kommunikation zwischen Arzt und Patient und die weitere Betreuung während einer laufenden Therapie sind von entscheidender Bedeutung (Anderson et al. 2007).

\subsubsection{Internetnutzung durch Patienten}

Bis zu $70 \%$ der amerikanischen Internetnutzer benutzen das Internet für medizinische Zwecke (Vinker et al. 2007). Im Jahr 2005 suchten mehr als ein Drittel der kanadischen Erwachsenen im Internet nach Gesundheitsinformationen (Underhill und Mckeown 2008) und 11 - $38 \%$ der Patienten holten vor einem Klinikaufenthalt Informationen aus dem Internet ein (Larner 2006, Pennekamp et al. 2006). Allerdings wurde nur von maximal einem Drittel die aus dem Internet erhaltene Information auch mit dem behandelnden Arzt diskutiert (Underhill und Mckeown 2008, Sim et al. 2007).

Einer der Hauptgründe für die Internetnutzung in der heutigen Zeit ist die schnelle und unkomplizierte Informationsgewinnung zu aller Art von Themen, darunter natürlich auch gesundheitliche Themen und Aspekte. Dieser bequeme Zugang zu Informationen stand auch bei den Nutzern des Expertenrates deutlich im Vordergrund. Von möglichen fünf Punkten (= trifft voll zu) erlangte dieser Aspekt 4,38 Punkte auf der Likert-Skala. Bei einer Umfrage von Umefjord et al. bei einem schwedischen Expertenrat zu allgemeinen Gesundheitsfragen wurde die Bequemlichkeit ebenfalls als häufigster Grund der Nutzung des Expertenrates angegeben (2003). In einer anderen Studie wurden vor allem unbeantwortet gebliebene Fragen nach einem Arztbesuch als häufigster Grund der Internetrecherche bei einem Gesundheitsportal angegeben (Delic et al. 2006).

Die meisten Anfragen der Expertenrat-Nutzer befassten sich mit den Themenkomplexen „Symptombeschreibung“ (32,2 \% der Anfragen) und „bestimmte Medikamente und Therapien" (32,0 \% der Anfragen). Andere Studien kamen bei der Analyse von Anfragen an Expertenräte mit unterschiedlichen Schwerpunktthemen zu ähnlichen Ergebnissen (Shuyler und Knight 2003, Arican 2007). Expertenforen bilden somit oft eine wichtige Anlaufstelle für Betroffene, wenn es darum geht, sich über Symptome oder Medikamente zu informieren, sei es, weil sie ungenügende Informationen durch den jeweiligen behandelnden Arzt erhalten haben oder sich mit ihren Problemen nicht primär in eine unmittelbare ärztliche Behandlung begeben möchten.

16,9 \% der Anfragen an den Kopfschmerz-Experten enthielten die Bitte um Hilfe und/ oder psychologischen Beistand. In den Anfragen wurde häufig die Ausweglosigkeit der betrof- 
fenen Patienten deutlich, die sich aufgrund ihrer Kopfschmerzen nicht mehr zu helfen wussten. Viele sahen im Expertenrat eine letzte Anlaufstelle und eine Art „letzte Instanz" bei der Suche nach Hilfe. So spielt das Internet neben seinen vielfältigen Informationsmöglichkeiten auch als Beratungsmedium eine bedeutende Rolle.

\subsubsection{Verbesserung der medizinischen Versorgung durch das Internet}

Viele der Expertenrat-Nutzer (33,3 \%) gaben bei der Verbesserung ihrer persönlichen, die Kopfschmerzen betreffenden Versorgungssituation durch den Expertenrat die weiterführende Informationsgewinnung und den damit zusammenhängenden Abbau von Unsicherheiten über die eigene individuelle Kopfschmerztherapie an. Auch hier wurde wieder der Aspekt der bequemen, schnellen und einfachen Informationsgewinnung angegeben. Zudem konnte vielen die gewonnene Information bei der Einschätzung der eigenen Situation helfen.

Es gibt in der Literatur kaum Studien, die die Zufriedenheit von Nutzern mit einem Internetforum untersuchen. Umefjord et al. untersuchten einen schwedischen „Ask-the-doctorservice“, bei dem die Nutzer im Allgemeinen zufrieden waren mit der erhaltenen Antworten (2006). 43 \% der Umfrageteilnehmer gaben hier an, dass die Fragen umfassend beantwortet worden seien und sie ausreichend Informationen durch die Antwort des Experten bekommen hätten. Eine Studie über eine Internetseite mit Informationen und einem Expertenforum zum Thema "Lungenkrebs" berichtete, dass $83 \%$ der Patienten mit der gewonnenen Information zufrieden waren (Linssen et al. 2007).

Internetexpertenforen könnten in ihrer Funktionsweise als Informationsmedium auch eine wichtige Rolle dahingehend einnehmen, indem sie Patienten möglicherweise an eine ärztliche Behandlung heranführen und Verbindungen zwischen niedergelassenen Ärzten und Patienten aufbauen. Dies könnte zum Beispiel dadurch geschehen, dass Patienten, die sich mit ihren medizinischen Problemen an Internetforen wenden, hier gezielt Kontaktdaten von entsprechenden Ärzten in ihrer häuslichen Umgebung erhalten und so eine individuelle weiterführende Therapie eingeleitet werden kann.

\subsection{Ausblick}

\subsubsection{Zukunft der Internetsprechstunden}

Das Internet ist aus dem alläglichen Leben nicht mehr wegzudenken und hält auch immer mehr Einzug in den medizinischen Praxisalltag. Auch wenn noch einige Aspekte im täglichen Umgang mit dem Internet geklärt werden müssen, wie z. B. rechtliche oder abrech- 
nungstechnische Angelegenheiten (Weiss 2004, Gottlieb 2004), so sind OnlineArztkonsultationen doch ein leistungsstarkes Instrument für Patienten und Ärzte (Grazi 2006), welches die Kommunikation zwischen beiden Parteien erleichtern kann (Freed 2003). Es sollten von Seiten der Ärzte als mögliche Anbieter von Internetsprechstunden und der Gesundheitspolitik her Maßnahmen getroffen werden, um das Internet mehr und mehr in den klinischen Alltag zu integrieren und den Patienten neue Leistungen anbieten zu können. Hierbei sind derartige Online-Sprechstunden nicht als Ersatz, sondern vielmehr als Ergänzung zum „konventionellen“ Arztbesuch zu sehen (Umefjord et al. 2004). Internet- und/ oder E-Mail-gestützte Beratung kann einen festen Bestandteil der ArztPatient-Beziehung der Zukunft bilden (Umefjord et al. 2003). Gerade in der Betreuung chronisch erkrankter Patienten tun sich hier neue Möglichkeiten der Kommunikation auf, welche die versorgenden Arztpraxen entlasten können. So ist es vorstellbar, dass weniger dringliche Anfragen wie z. B. die Ausstellung von Folgerezepten oder einfache Fragen an den behandelnden Arzt immer mehr über E-Mail-Kontakt abgewickelt werden (Neville et al. 2004 b). Die Arzt-Patienten-Beziehung wird hierbei durchaus positiv gefördert, da die Patienten das Internet als eine zusätzliche, unterstützende Quelle von Informationen sehen (Stevenson et al. 2007). Die Anonymität des Internets erleichtert den ersten Zugang zu medizinischer Information gerade bei weniger angenehmen oder sensibel belegten Themen. Allerdings besteht durch die Vielzahl der im Internet dargebotenen Informationen die Gefahr der Fehlinformation oder der Missinterpretation von erlangten Informationen, so dass hier Instrumente zur Qualitätsmessung von Internetseiten sinnvoll erscheinen. Denkbar wäre auch die Einführung eines Gütesiegels, das geprüfte Internetseiten für die Internetnutzer erkennbar macht. Zu vermuten ist, dass vor allem diejenigen Patienten medizinische Internetangebote nutzen, deren Bedürfnisse im regulären Gesundheitssystem nicht angemessen zu befriedigen sind (Umefjord et al. 2003). Doch gerade bei diesen Patienten bietet das Internet dann die Möglichkeit, unerfüllt gebliebene Wünsche und Bedürfnisse zu erfüllen.

Neben den Internetsprechstunden bietet das Internet aber noch weitere Möglichkeiten der medizinischen Betreuung von Patienten. So wurde in Studien bereits über positive Erfahrungen mit z. B. internetbasierter Verhaltenstherapie oder Entspannungstechniken und Problemlösung bei chronischen Kopfschmerzen berichtet (Devineni und Blanchard 2005, Strom et al. 2000). Doch nicht nur das Internet, auch andere Medien bieten weiteres Potential in der Verbesserung der Patientenversorgung. So wurde von Farmer et al. ein Mobiltelefon-gestütztes Telemedizinsystem zur Überwachung von Blutglucosespiegeln bei jungen Typ-1-Diabetikern entwickelt (2005). 


\subsubsection{Mögliche Verbesserung der hausärztlichen Versorgung}

Chronische Schmerzpatienten (und somit auch Kopfschmerzpatienten) bleiben eine Herausforderung für den behandelnden Hausarzt im klinischen Alltag. Häufig bleibt für lange Anamnesegespräche und ausführliche Beratung im straff organisierten Praxisalltag nicht ausreichend Zeit. Hier sind die Hausärzte gefordert, trotz der knapp bemessenen Zeit empathisch auf die Patienten zuzugehen und Verständnis für die jeweilige Situation des Patienten zu zeigen. Dabei sollte das weitere Vorgehen oder die Therapie zusammen mit dem Patienten festgelegt und besprochen werden, um dem Patienten die Möglichkeit zu geben, rechtzeitig Fragen zu stellen und Bedenken zu äußern.

Zudem sollte der Umgang mit chronisch kranken Patienten fester Bestandteil der medizinischen Ausbildung sein. In einer amerikanischen Umfrage gaben Ärzte an, ihre Ausbildung im Umgang mit chronisch Kranken sei nicht ausreichend und eine bessere Ausbildung im Hinblick hierauf sei nötig (Darer et al. 2004). In England wurde der Ausdruck eines „Allgemeinarztes mit speziellen Interessen“ eingeführt, der eine zusätzliche Ausbildung in einem Spezialgebiet beinhaltet (Ridsdale et al. 2008). Hierbei ist auch die Spezialisierung auf die Versorgung von Kopfschmerzpatienten möglich. Im Vergleich mit Neurologen zeigte sich eine deutlich höhere Zufriedenheit und Symptomfreiheit der behandelten Patienten. Zudem waren die Kosten der jeweiligen Arztbesuche niedriger als die der neurologischen Konsultationen. Dies scheint eine zusätzliche Möglichkeit der speziellen Betreuung von Kopfschmerzpatienten neben den Schmerzkliniken zu sein, die durch ihren Aufbau und den meist erforderlichen stationären Aufenthalt ungleich kostenintensiver sind. 


\section{Zusammenfassung}

Hintergrund: Kopfschmerzen sind ein häufiger Konsultationsgrund in der hausärztlichen Praxis und können in den unterschiedlichsten Formen und Zusammenhängen auftreten. Besonders Migräne und chronische Spannungskopfschmerzen haben einen großen Einfluss auf die Lebensqualität und den Alltag der betroffenen Patienten. Dennoch werden Kopfschmerzen nur von einem Teil der Betroffenen als Beratungsanlass in Hausarztpraxen thematisiert. Es ist zu vermuten, dass bei den betroffenen Kopfschmerzpatienten eine hohe Arztverdrossenheit und Unzufriedenheit mit der jeweiligen Behandlung der Kopfschmerzen herrscht. Die hausärztliche Betreuung von chronischen Kopfschmerzpatienten ist aufwendig und erfordert neben viel Engagement auch einen großen Zeitaufwand des behandeInden Hausarztes. Alternative Konsultationsformen wie Telemedizin oder Internetforen gewinnen zunehmend an Bedeutung.

Fragestellung: In der vorliegenden Arbeit sollten die Beweggründe analysiert werden, die Betroffene zum Besuch des Internets veranlassen. Zudem sollte die Zufriedenheit mit der individuellen ärztlichen Versorgung im Bezug auf die Kopfschmerzsymptomatik untersucht werden.

Methoden: Unter den Nutzern eines Kopfschmerz-Expertenrates im Internet wurde eine Onlinebefragung mittels eines standardisierten Fragebogens durchgeführt und sämtliche Anfragen, die an den Expertenrat gestellt wurden, auf ihren Inhalt hin untersucht. Die Nutzer des Expertenrats wurden zu ihren persönlichen Gründen, den Expertenrat zu besuchen, befragt und gebeten, ihre Erfahrungen mit den jeweiligen behandelnden Ärzten zu bewerten. Bei der inhaltsanalytischen Untersuchung der Anfragen wurden neben statistischen Daten vor allem die jeweils angesprochenen Themen erhoben.

Ergebnisse: Es zeigte sich, dass der Hauptteil der Nutzer den Expertenrat hauptsächlich aufgrund seiner Möglichkeit der schnellen und unkomplizierten Informationsgewinnung schätzt. In der Analyse der Anfrage fanden sich jedoch auch viele Beispiele, in denen verzweifelte chronische Kopfschmerzerkrankte nach Hilfe suchten. Diese Betroffenen befanden sich teilweise in einer für sie ausweglos erscheinenden Situation und sahen im Expertenrat vielfach die letzte Anlaufstelle für ihre Problematik. In den Anfragen an den Expertenrat wurde zumeist nach Symptomen z. B. von Migräne oder nach bestimmten Therapien und Medikamenten gefragt. 
Schlussfolgerung: Chronische Kopfschmerzpatienten stellen ein Patientenkollektiv dar, welches ein besonderes Augenmerk in der hausärztlichen Versorgung verdient hat. Die medizinische Betreuung dieser Patienten fordert vom behandelnden Arzt häufig viel Empathie und Verständnis, da oftmals von Seiten der Betroffenen Anforderungen gestellt werden, die über das übliche Maß hinausgehen. Dies lässt sich hauptsächlich mit der durch Kopfschmerzen verursachten starken Beeinträchtigung des Einzelnen im alläglichen Leben erklären. In der Betreuung von Patienten spielt das Internet eine stetig zunehmende Rolle. Hierbei ist es jedoch nicht als Ersatz der individuellen persönlichen medizinischen Betreuung zu sehen, sondern stellt vielmehr eine Ergänzung des klinischen Alltags dar. 


\section{Anhang}

\subsection{Anfrage zur Teilnahme an der Internetumfrage}

\section{Liebe Forumsteilnehmerin, lieber Forumsteilnehmer!}

Vielen Dank für Ihre Anfrage, die der Experte Dr. Mildenstein so schnell wie möglich beantworten wird.

Unabhängig von BSMO (der Betreibergesellschaft des Expertenrats), führt die Abteilung Allgemeinmedizin der Universität Göttingen eine Studie durch. Diese Studie wird freundlicherweise von BSMO und Dr. Mildenstein unterstützt. Der Rat des Experten spiegelt aber nicht unbedingt die Meinung der Universität Göttingen wider. Wir möchten herausfinden, welche Rolle das Internet bei der Behandlung von Kopfschmerzen spielt und in wieweit es möglicherweise Versäumnisse niedergelassener Ärzte ausgleicht. Sie können uns dabei helfen, wichtige Erkenntnisse zur Verbesserung der Betreuung von Patienten mit Kopfschmerzen zu gewinnen.

Wenn Sie Interesse haben, an der Studie teilzunehmen und mehr über uns wissen möchten, erhalten Sie hier weitere Informationen über die Verantwortlichen dieser Studie. Die Beantwortung des Fragebogens wird ca. 10 Minuten dauern. Selbstverständlich verläuft die Beantwortung der Fragen anonym. Es ist uns zu keiner Zeit möglich, auf Ihre Person zurückzuschließen. Außerdem garantieren wir dafür, dass I hre Angaben nicht durch Dritte eingesehen werden können. Bitte nehmen Sie nur einmal an der Befragung teil, auch wenn beim erneuten Abschicken einer Frage an den Expertenrat diese Aufforderung nochmals erscheinen sollte.

Ob Sie den Fragebogen ausfüllen oder nicht: Sie erhalten in jedem Fall eine Antwort auf Ihre Frage. Aber bitte bedenken Sie, dass I hnen und anderen Betroffenen die Ergebnisse unserer Untersuchung zugute kommen können.

Ich habe die Informationen verstanden und möchte an der Studie teilnehmen

$\mathrm{Ja}=>$ Weiterleitung an FB

Nein $=>$ Weiterleitung zum Forum

\section{Herzlichen Dank für I hre Mitarbeit!}

Rufen Sie bei Fragen Dr. Martin Scherer unter 0551-39-14227 an. Auf der Homepage http://www. allgemeinmedizin.med.uni-goettingen.de/ finden Sie weitere Informationen über uns! 


\subsection{Internet-Fragebogen (Kieler Fragebogen ab Frage 20)}

Hatten Sie in den letzten sechs Monaten keine Kopfschmerzen?

Wenn das für Sie zutrifft, ist die Befragung für Sie damit beendet.

Klicken Sie bitten auf den Button „kein Kopfweh“.

Sie werden dann zum Forum zurückgeleitet.

1. Haben Sie seit Juli 2005 eine oder mehrere Fragen an Dr. Mildenstein im Expertenrat „Kopfschmerz und Migräne“ gestellt?

$$
\text { ja ( ) nein ( ) }
$$

2. Falls Sie sich noch daran erinnern, können Sie uns das Thema der Frage nennen?

(Freitextantwort)

3. Wie haben Sie von dieser Seite erfahren?

Empfehlung vom behandelnden Arzt

Durch andere Betroffene

Durch Familie

Durch Freunde.

Durch gezieltes Suchen in einer Suchmaschine.

Zufällig durch Surfen im Internet

Andere

4. Seit wann nutzen Sie den Expertenrat?

Seit Wochen

5. Haben Sie schon einmal andere Internetsprechstunden genutzt?

$$
\text { ja ( ) nein ( ) }
$$

6. Wie häufig benutzen Sie Internetsprechstunden?

Täglich....

Wöchentlich

Monatlich

Weniger als zwölf Mal pro Jahr...( ) 
7. Wie oft nutzen Sie das Internet, um Informationen zu Kopfschmerzen zu bekommen?

Täglich.....

Wöchentlich.

Monatlich....

Weniger als zwölf Mal pro Jahr...( )

8. Für welche Bereiche nutzen Sie das Internet außerdem noch?

Allgemeine Informationen (z. B. Nachrichten)....( )

Einkaufen.

Reisebuchung

Chat, Besuch von Foren.

Online-Banking

Andere

9. Haben Sie einen Hausarzt?

$$
\text { ja ( ) nein ( ) }
$$

10. Welche Gründe haben Sie persönlich, den Expertenrat aufzusuchen?

$$
\begin{gathered}
\text { trifft überhaupt } \\
\text { nicht zu }
\end{gathered}
$$

Geringer zeitlicher Aufwand....

(1) (2)

(3) (4)

Anonymität....

(2)

(3) (4)

Wenig Aufwand

(3) (4)

Kontakt zu anderen Betroffenen

(2)

(3) (4)

Kein persönliches Vertrauen

zum behandelnden Arzt. . .

(3) (4)

(5)

Mangelnde Kompetenz

des behandelnden Arztes.

(1) (2)

(3) (4)

(5)

Andere

(2)

(3)

(4)

(5) 
11. Waren Sie aufgrund Ihrer Kopfschmerzen in den letzten sechs Monaten schon einmal bei einem Arzt?

\begin{tabular}{|c|c|c|}
\hline ja & nein & wenn ja, wie oft? \\
\hline Hausarzt...................................... ( ) & () & $-{ }_{-}$Mal \\
\hline Neurologe $\ldots \ldots \ldots \ldots \ldots \ldots \ldots \ldots \ldots \ldots \ldots \ldots \ldots \ldots \ldots \ldots \ldots \ldots \ldots \ldots$ & () & __ Mal \\
\hline Augenarzt........................................ ( ) & () & $-{ }_{-}$Mal \\
\hline Orthopäde.................................. ( ) & () & _- Mal \\
\hline Internist........................................ ( ) & ( ) & $-{ }_{-}$Mal \\
\hline HNO-Arzt...................................... ( ) & ( ) & _- Mal \\
\hline Heilpraktiker.....................................( ) & () & $-{ }_{-}$Mal \\
\hline Chirotherapeut................................. ( ) & () & __ Mal \\
\hline
\end{tabular}

12. Wenn nicht, haben Sie vor, noch einen Arzt aufzusuchen?

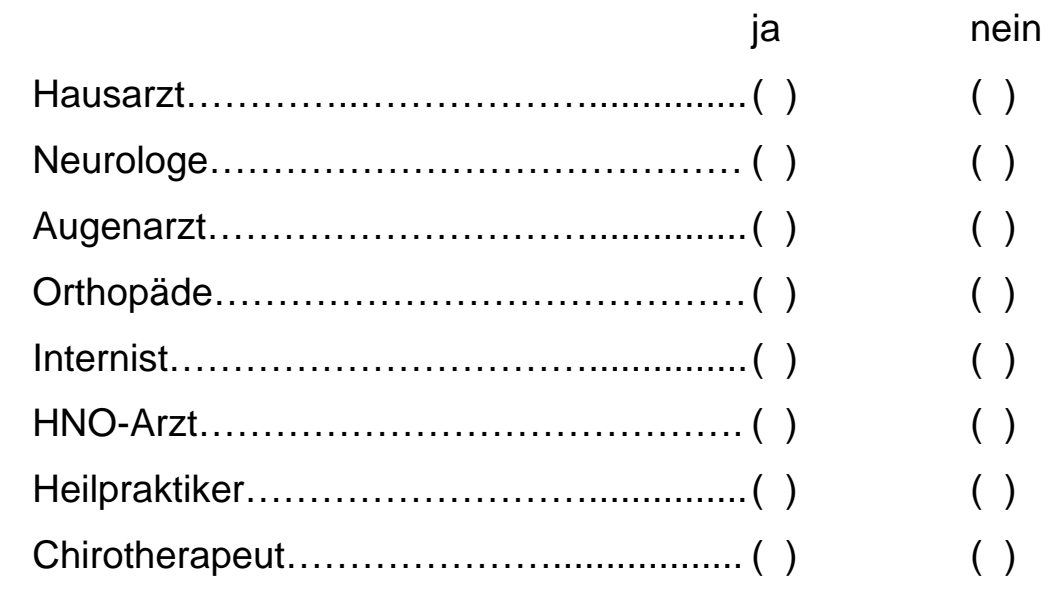

13. Welche Behandlungen haben Sie bis jetzt erhalten?

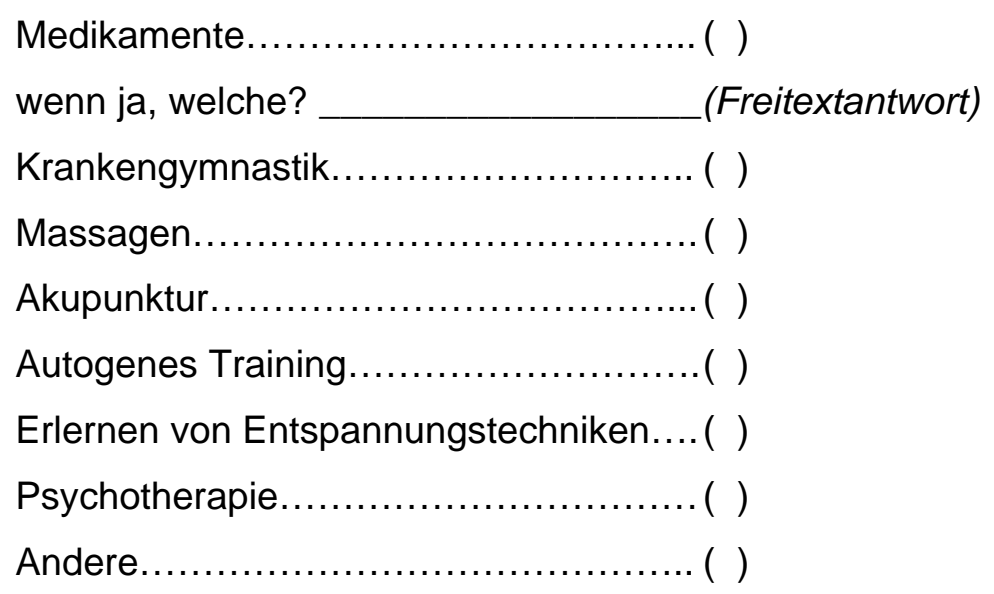


14. Wie zufrieden sind Sie mit der Behandlung Ihrer Kopfschmerzen durch Ihren...?

\section{Überhaupt nicht zufrieden}

Hausarzt

Neurologen

Augenarzt

Orthopäden

Internisten.

HNO-Arzt

Heilpraktiker

Chirotherapeuten
(1) (2)

(1) (2)

(1) (2)

(1) (2)

(1) (2)

(1) (2)

(1) (2)

(1) (2) vollkommen zufrieden
(3) (4) (5)

(3) (4)

(3) (4)

(3) (4)

(3) (4)

(3) (4)

(3) (4)

15. Wenn Sie nicht zufrieden sind, was stört Sie bzw. was fehlt Ihnen (bitte geben Sie an, auf welchen Arzt Sie sich beziehen: Neurologen, Augenarzt etc.)?

(Freitextantwort)

16. Inwieweit bringt das Internet bezüglich der oben genannten Probleme eine Verbesserung?

(Freitextantwort)

17. Wie zufrieden sind Sie mit den Antworten des Experten vom Expertenrat?
Überhaupt nicht

zufrieden

(1) (2)

(3) (4)

(5)


20. Treten bei Ihnen Kopfschmerzen auf, die so oder ähnlich aussehen?

Dauer ohne Behandlung: 4 bis 72 Stunden....

Anfallsweises Auftreten

Zwischen den Anfällen keine Kopfschmerzen

Einseitiges Auftreten

Pochender, pulsierender oder hämmernder Schmerz

Übelkeit, Erbrechen, Lärm- oder Lichtempfindlichkeit

können den Schmerz begleiten.

Falls bei Ihnen solche oder ähnliche Kopfschmerzen auftreten, beantworten Sie bitte die folgenden Fragen.

(Treten solche Kopfschmerzen bei Ihnen nicht auf, setzen Sie die Beantwortung bitte bei Frage 33 fort.)

21. Dauern diese Kopfschmerzanfälle 4 bis 72 Stunden an, wenn Sie kein Medikament einnehmen oder eine Behandlung erfolglos bleibt?

$$
\text { ja ( ) nein ( ) }
$$

22. Können sich diese Kopfschmerzen auf eine Kopfhälfte beschränken?

$$
\text { ja ( ) nein ( ) }
$$

23. Können diese Kopfschmerzen einen pulsierenden Charakter haben?

$$
\text { ja ( ) nein ( ) }
$$

24. Können diese Kopfschmerzen Ihre übliche Tätigkeit erheblich beeinträchtigen?

$$
\text { ja ( ) nein ( ) }
$$

25. Können diese Kopfschmerzen beim Treppensteigen oder durch andere körperliche Aktivität verstärkt werden?

$$
\text { ja ( ) nein ( ) }
$$

26. Können diese Kopfschmerzen von Übelkeit begleitet werden?

$$
\text { ja ( ) nein ( ) }
$$

27. Können diese Kopfschmerzen von Erbrechen begleitet werden?

$$
\text { ja ( ) nein ( ) }
$$

28. Können diese Kopfschmerzen von Lichtempfindlichkeit begleitet werden?

$$
\text { ja ( ) nein ( ) }
$$

29. Können diese Kopfschmerzen von Lärmempfindlichkeit begleitet werden?

$$
\text { ja ( ) nein ( ) }
$$

30. Sind bei Ihnen schon mindestens fünf Kopfschmerzanfälle aufgetreten, die der Beschreibung entsprechen?

$$
\text { ja ( ) nein ( ) }
$$


31. Wie lange leiden Sie an solchen Kopfschmerzanfällen? Geben Sie bitte die entsprechende Anzahl in Jahren an: Jahre

32. An wie vielen Tagen pro Monat leiden Sie durchschnittlich an entsprechenden Kopfschmerzanfällen? Geben Sie bitte die Anzahl der Tage pro Monat an: Tage

33. Treten bei Ihnen Kopfschmerzen auf, die man wie folgt beschreiben kann?

Dauer ohne Behandlung: 30 Minuten bis 7 Tage....... ( )

Beidseitiges Auftreten.............................. ( )

Kann anfallsweise oder täglich auftreten................ ( )

Drückender, ziehender, dumpfer Schmerz............. ( )

Kein Erbrechen oder Übelkeit.........................( )

Falls bei Ihnen solche oder ähnliche Kopfschmerzen auftreten, beantworten Sie bitte die folgenden Fragen.

(Treten solche Kopfschmerzen bei Ihnen nicht auf, ist die Befragung abgeschlossen.)

34. Dauern diese Kopfschmerzen gewöhnlich 30 Minuten bis 7 Tage an, wenn Sie kein Medikament einnehmen oder eine Behandlung erfolglos bleibt?

$$
\text { ja ( ) nein ( ) }
$$

35. Können diese Kopfschmerzen einen dumpfen, drückenden bis ziehenden Charakter haben?

$$
\text { ja ( ) nein ( ) }
$$

36. Können Sie trotz dieser Kopfschmerzen Ihrer üblichen Tagesaktivität nachgehen?

$$
\text { ja ( ) nein ( ) }
$$

37. Können diese Kopfschmerzen bei Ihnen beidseitig auftreten?

$$
\text { ja ( ) nein ( ) }
$$

38. Bleiben diese Kopfschmerzen durch körperliche Aktivität (z. B. Treppensteigen) unbeeinflusst?

$$
\text { ja ( ) nein ( ) }
$$

39. Können diese Kopfschmerzen von Übelkeit begleitet werden?

$$
\text { ja ( ) nein ( ) }
$$

40. Können diese Kopfschmerzen von Erbrechen begleitet werden?

$$
\text { ja ( ) nein ( ) }
$$

41. Können diese Kopfschmerzen von Lichtempfindlichkeit begleitet werden?

$$
\text { ja ( ) nein ( ) }
$$


42. Können diese Kopfschmerzen von Lärmempfindlichkeit begleitet werden?

$$
\text { ja ( ) nein ( ) }
$$

43. Sind bei Ihnen schon mindestens zehn Kopfschmerzanfälle aufgetreten, die der angegebenen Beschreibung gleichen?

$$
\text { ja ( ) nein ( ) }
$$

44. An wie vielen Tagen pro Monat leiden Sie durchschnittlich an solchen Kopfschmerzanfällen? Geben Sie bitte die entsprechende Anzahl an:

$$
\text { Tage }
$$

45. Leiden Sie schon länger an solchen Kopfschmerzen?

$$
\text { ja ( ) nein ( ) }
$$

46. Seit wie vielen Jahren leiden Sie an solchen Kopfschmerzen? Geben Sie bitte die entsprechende Zahl an:

Jahre

\section{Angaben zu Ihrer Person:}

47. Ihr Geschlecht? weiblich ( ) männlich ( )

48. Welche Staatsangehörigkeit haben Sie? (Freitextantwort)

49. Wann sind Sie geboren? Monat Jahr

50. Leben Sie mit einem festen Partner zusammen?. .ja ( ) nein ( )

51. Wie viele Personen leben ständig in Ihrem Haushalt, Sie selbst eingeschlossen? Insgesamt Personen 


\subsection{Grundauszählung des Internet-Fragebogens}

Durch das Runden der Prozentzahlen können Rundungsfehler auftreten.

Die Aufzählung der Fragen richtet sich nach der Reihenfolge im Online-Fragebogen. Freitextangaben werden nicht aufgeführt.

\section{Sondierungsfrage $A$}

\begin{tabular}{lccc}
$\begin{array}{l}\text { Hatten Sie in den letzten sechs Monaten keine } \\
\text { Kopfschmerzen? }\end{array}$ & Anzahl (n) & Prozent (\%) \\
\hline$-\quad$ Ja & 10 & $\begin{array}{l}16,4 \\
83,6\end{array}$ \\
$-\quad$ Nein & 51 & 100,0 \\
\hline Gesamt & 61 & \\
\hline
\end{tabular}

\section{Sondierungsfrage $B$}

Haben Sie seit Juli 2005 eine oder mehrere

Fragen an Dr. Mildenstein im Expertenrat

„Kopfschmerz und Migräne“ gestellt?

Anzahl (n) Prozent (\%)

\begin{tabular}{clcc}
\hline Ja & 39 & 76,5 \\
$-\quad$ Nein & 12 & 23,5 \\
\hline \multirow{2}{*}{ Gesamt } & 51 & 100,0 \\
\hline
\end{tabular}

Frage 1

Wie haben Sie von dieser Seite erfahren?

(Mehrfachantworten möglich)

Anzahl (n)

Prozent (\%)

- Empfehlung vom behandelnden Arzt

0

- Durch andere Betroffene

0

5,1

- Durch Familie

- Durch Freunde

2

0

- Durch gezieltes Suchen in einer

Suchmaschine

0

0

- Zufällig durch Surfen im Internet

61,5

13

33,3 


\section{Frage 3}

\begin{tabular}{|c|c|c|c|c|}
\hline $\begin{array}{l}\text { Seit wann nutzen Sie } \\
\text { den Expertenrat? } \\
\text { (Angaben in Wochen) }\end{array}$ & Mittel & Minimum & Maximum & SD \\
\hline & 15,26 & 0 & 99 & 28,766 \\
\hline \multicolumn{5}{|l|}{ Missings $=0$} \\
\hline \multicolumn{5}{|l|}{ Frage 5} \\
\hline \multicolumn{5}{|l|}{ Haben Sie schon einmal andere } \\
\hline Internetsprechstunden genutzt? & & Anzahl (n) & \multicolumn{2}{|c|}{ Prozent (\%) } \\
\hline - Ja & & 15 & \multicolumn{2}{|c|}{38,5} \\
\hline - $\quad$ Nein & & 22 & \multicolumn{2}{|c|}{56,4} \\
\hline - Keine Angabe & & 2 & \multicolumn{2}{|c|}{5,1} \\
\hline Gesamt & & 39 & \multicolumn{2}{|c|}{100,0} \\
\hline
\end{tabular}

Frage 6

\begin{tabular}{llll}
\hline Wie häufig nutzen Sie Internetsprechstunden? & Anzahl (n) & Prozent (\%) \\
\hline - Täglich & 2 & 5,1 \\
- Wöchentlich & 6 & 15,4 \\
- Monatlich & 4 & 10,3 \\
- Weniger als zwölf Mal pro Jahr & 24 & 61,5 \\
- Keine Angabe & 3 & 7,7 \\
\hline Gesamt & 39 & 100,0 \\
\hline
\end{tabular}

\section{Frage 7}

Wie oft nutzen Sie das Internet, um

Informationen über Kopfschmerzen zu

bekommen?

Anzahl (n) $\quad$ Prozent (\%)

- Täglich

- Wöchentlich

- Monatlich

- Weniger als zwölf Mal pro Jahr

- Keine Angabe

$6 \quad 15,4$

$10 \quad 25,6$

$6 \quad 15,4$

$16 \quad 41,0$

12,6

Gesamt 


\section{Frage 8}

Für welche Bereiche nutzen Sie das Internet außerdem noch?

(Mehrfach-Antwort möglich)

Anzahl (n)

Prozent (\%)

- Allgemeine Informationen (z. B. Nachrichten)

31

79,5

- Einkaufen

21

53,8

- Reisebuchung

18

46,2

- Chat, Besuch von Foren

- Online-Banking

- Andere

9

23,1

\section{Frage 9}

\begin{tabular}{cccc}
\hline Haben Sie einen Hausarzt? & Anzahl (n) & Prozent (\%) \\
\hline Ja & 36 & 92,3 \\
$-\quad$ Nein & 2 & 5,1 \\
$-\quad$ Keine Angabe & 1 & 2,6 \\
\hline Gesamt & 39 & 100,0 \\
\hline
\end{tabular}

\section{Frage 10}

Welche Gründe haben Sie persönlich, den Expertenrat aufzusuchen?

Mittel

Minimum Maximum SD

- Zeitersparnis

3,24

2,61

- Anonymität

- $\quad$ Bequemer Informationszugang 4,38

- Kein persönliches Vertrauen 2,03 zum behandelnden Arzt

- Mangelnde Kompetenz des $\quad 2,24$

$2,24 \quad 1$

1

1

1

1

1

SD
behandelnden Arztes

- Andere

3,04

1

5

1,211

1 = trifft überhaupt nicht zu, 5 = trifft voll zu 


\section{Frage 11}

Waren Sie aufgrund Ihrer Kopfschmerzen in den letzten sechs Monaten schon einmal bei einem Arzt? (Mehrfachantwort möglich)

Anzahl (n)

Prozent (\%)

- Hausarzt

32

- Neurologe

20

82,1

- Augenarzt

14

- Orthopäde

15

Internist

9

- HNO-Arzt

12

51,3

Heilpraktiker

9

35,9

38,5

23,1

- Chirotherapeut

6

23,1

15,4

\section{Frage 11 a}

\begin{tabular}{lllll}
\hline $\begin{array}{l}\text { Wenn ja, wie oft waren Sie schon } \\
\text { beim Arzt? }\end{array}$ & Mittel & Minimum & Maximum & SD \\
\hline - Hausarzt & 3,81 & 1 & 30 & 5,706 \\
- Neurologe & 3,33 & 1 & 18 & 4,304 \\
- Augenarzt & 1,27 & 1 & 3 & 0,647 \\
- Orthopäde & 1,64 & 1 & 4 & 1,151 \\
- Internist & 1,50 & 1 & 2 & 0,577 \\
- HNO-Arzt & 1,75 & 1 & 5 & 1,357 \\
- Heilpraktiker & 5,0 & 1 & 12 & 4,050 \\
- Chirotherapeut & 2,33 & 1 & 4 & 1,528 \\
\hline
\end{tabular}

Frage 12

Wenn nicht, haben Sie noch vor, einen

Arzt aufzusuchen?

Anzahl (n)

Prozent (\%)

- Hausarzt

11

28,2

- Neurologe

6

15,4

- Augenarzt

5

12,8

- Orthopäde

4

- Internist

- HNO-Arzt

0

10,3

- Heilpraktiker

4

0,0

- Chirotherapeut

6

10,3

15,4

$3 \quad 7,7$ 


\section{Frage 13}

Welche Behandlungen haben Sie

bisher erhalten?

Anzahl (n)

Prozent (\%)

- Medikamente

31

79,5

- Krankengymnastik

14

35,9

- Massagen

13

33,3

- Akupunktur

16

41,0

- Autogenes Training

8

20,5

- Erlernen von Entspannungstechniken

12

30,8

- Psychotherapie

- Andere

7

17,9

7,7

\section{Frage 13 a}

Welche Medikamente haben Sie bisher

eingenommen?

Anzahl (n)

Prozent (\%)

Akutmedikation:

- Triptane

46,2

- NSAR (auch Kombipräparate)

14

- Antiemetika (Metoclopramid/ Dimenhydrinat)

- Metamizol/ Phenazon

- Benzodiazepine

- Tramadol

- Ergotamin

- Katadolon

- Opioide

Anfallsprophylaxe:

- Betablocker

- Antidepressiva

- Magnesium

- Pestwurzpräparat

- Calciumantagonisten

- ACE-Hemmer

$2 \quad 5,1$

$2 \quad 5,1$

- Homöopathie 


\section{Frage 14}

Wie zufrieden sind Sie mit der

Behandlung durch Ihren...

Mittel Minimum Maximum SD

\begin{tabular}{lllll}
\hline - Hausarzt & 2,63 & 1 & 5 & 1,338 \\
- Neurologen & 2,15 & 1 & 5 & 1,348 \\
- Augenarzt & 2,43 & 1 & 5 & 1,158 \\
- Orthopäden & 2,60 & 1 & 5 & 1,549 \\
- Internisten & 2,11 & 1 & 5 & 1,453 \\
- HNO-Arzt & 2,27 & 1 & 4 & 1,104 \\
- Heilpraktiker & 3,00 & 1 & 5 & 1,732 \\
- Chirotherapeuten & 2,33 & 1 & 4 & 1,366
\end{tabular}

1 = überhaupt nicht zufrieden, 5 = sehr zufrieden

\section{Frage 17}

Wie zufrieden sind Sie mit den

Antworten des Experten

vom Expertenrat? Mittel Minimum Maximum SD

\begin{tabular}{lllll}
\hline 4,08 & 1 & 5 & 1,055 \\
\hline
\end{tabular}

1 = überhaupt nicht zufrieden, 5 = sehr zufrieden

\section{Frage 18}

\begin{tabular}{llccc}
\hline $\begin{array}{l}\text { Fühlen Sie sich mit Ihren } \\
\text { Beschwerden ernst genommen? }\end{array}$ & Mittel & Minimum & Maximum & SD \\
\hline - Durch Familie & 4,16 & 1 & 5 & 1,175 \\
- Durch enge Freunde & 3,68 & 1 & 5 & 1,165 \\
- Durch Bekannte & 3,19 & 1 & 5 & 1,238 \\
- Durch Arbeitskollegen & 2,73 & 1 & 5 & 1,126 \\
- Durch den behandelnden Arzt & 3,34 & 1 & 5 & 1,169 \\
\hline
\end{tabular}

1 = überhaupt nicht ernst genommen, 5 = sehr ernst genommen 


\section{Frage 19}

Welche zusätzlichen Erkrankungen außer den Kopfschmerzen sind bei Ihnen bekannt?

Anzahl (n)

Prozent (\%)

- Schilddrüsenerkrankung

- Hypertonus

- Augenerkr. (z. B. Schielen, Fehlsichtigkeit)

\section{4}

3

3

1

- Rezidivierende Blasenentzündungen

- Beinlängendifferenz, Beckenschiefstand; Rücken- und Nackenschmerzen als Folge

- Supraspinatussyndrom

- Hypotonus

- Verspannung der HWS durch Fehlhaltung

- Bulimie, Auto-Aggression, Depression im Rahmen einer Borderline-Störung

- Epilepsie

- Polyzystisches Ovar-Syndrom (PCOS)

- Osteochondrosis dissecans

- Innenohrschwerhörigkeit

- Encephalomyelitis disseminata (Multiple Sklerose)

- Panikattacken

- Herzrhythmusstörungen

- Magenprobleme

- Klimakterium praecox

- Leichte Venenschwäche
2

2

2

2

1

10,3

7,7

7,7

5,1

5,1

5,1

5,1

2,6

2,6

1

2,6

2,6

2,6

2,6

1

2,6

2,6

2,6

2,6

12,6

12,6

12,6

12,6

12,6

12,6

12,6

\section{Frage 20}

Treten bei Ihnen Kopfschmerzen auf, die Antwort ,ja“ so oder ähnlich aussehen? Anzahl (n)

Prozent (\%)

a) Dauer ohne Behandlung: 4-72 Stunden

32

27

c) Zwischen den Anfällen keine Kopfschmerzen

d) Einseitiges Auftreten

14

29

28

28
82,1

69,2

35,9

74,4

71,8

71,8

Die Fragen 21 - 32 sollten nur von denjenigen Nutzern beantwortet werden, die auf die Fragen 20 a - f zumindest einmal mit ,ja“ geantwortet hatten. 


\section{Frage 21}

Dauern diese Kopfschmerzen 4 -72 Std. an, wenn

Sie kein Medikament einnehmen oder eine

Behandlung erfolglos bleibt?

Anzahl (n) $\quad$ Prozent (\%)

\begin{tabular}{llll}
\hline - Ja & 34 & 87,2 \\
- & Nein & 1 & 2,6 \\
- & 4 & 10,3 \\
\hline Geine Angabe & 39 & 100,1 \\
\hline
\end{tabular}

\section{Frage 22}

\begin{tabular}{lcc}
\hline $\begin{array}{l}\text { Können sich diese Kopfschmerzen auf eine } \\
\text { Kopfhälfte beschränken? }\end{array}$ & Anzahl (n) & Prozent (\%) \\
\hline - Ja & 30 & 76,9 \\
$-\quad$ Nein & 5 & 12,8 \\
$-\quad$ Keine Angabe & 4 & 10,3 \\
\hline Gesamt & 39 & 100,0 \\
\hline
\end{tabular}

\section{Frage 23}

Können diese Kopfschmerzen einen

pulsierenden Charakter haben?

Anzahl (n) $\quad$ Prozent (\%)

- Ja

- Nein

29

74,4

- Keine Angabe

4

10,3

6

15,4

Gesamt

100,1

\section{Frage 24}

Können diese Kopfschmerzen Ihre übliche

Tätigkeit erheblich beeinträchtigen?

Anzahl (n) $\quad$ Prozent (\%)

\begin{tabular}{llll}
\hline - Ja & 37 & 94,9 \\
- Nein & 0 & 0 \\
- Keine Angabe & 2 & 5,1 \\
\hline Gesamt & 39 & 100,0
\end{tabular}




\section{Frage 25}

\begin{tabular}{lcc}
\hline $\begin{array}{l}\text { Können diese Kopfschmerzen beim Treppen- } \\
\text { steigen oder durch andere körperliche Aktivität } \\
\text { verstärkt werden? }\end{array}$ & Anzahl (n) & Prozent (\%) \\
\hline - Ja & 30 & 76,9 \\
- Nein & 6 & 15,4 \\
- Keine Angabe & 3 & 7,7 \\
\hline Gesamt & 39 & 100,0 \\
\hline
\end{tabular}

\section{Frage 26}

Können diese Kopfschmerzen von Übelkeit begleitet werden?

Anzahl (n) Prozent (\%)

\begin{tabular}{llll}
\hline - Ja & 26 & 66,7 \\
- & Nein & 9 & 23,1 \\
- Keine Angabe & 4 & 10,3 \\
\hline Gesamt & 39 & \multirow{2}{*}{100,1} \\
\hline
\end{tabular}

\section{Frage 27}

Können diese Kopfschmerzen von Erbrechen begleitet werden?

Anzahl (n) $\quad$ Prozent (\%)

\begin{tabular}{llll}
\hline - Ja & 21 & 53,8 \\
- Nein & 14 & 35,9 \\
- Keine Angabe & 4 & 10,3 \\
\hline \multirow{2}{*}{ Gesamt } & \multirow{2}{*}{39} & 100,0 \\
\hline
\end{tabular}

\section{Frage 28}

Können diese Kopfschmerzen von Lichtempfindlichkeit begleitet werden?

Anzahl (n) $\quad$ Prozent (\%)

\begin{tabular}{llll}
\hline - Ja & 24 & 61,5 \\
- Nein & 8 & 20,5 \\
- Keine Angabe & 7 & 17,9 \\
\hline Gesamt & 39 & 99,9 \\
\hline
\end{tabular}




\section{Frage 29}

Können diese Kopfschmerzen von Lärm-

empfindlichkeit begleitet werden?

Anzahl (n)

Prozent (\%)

\begin{tabular}{clll}
\hline - Ja & 29 & 74,4 \\
- Nein & 7 & 17,9 \\
- Keine Angabe & 3 & 7,7 \\
\hline Gesamt & 39 & 100,0
\end{tabular}

\section{Frage 30}

Sind bei Ihnen schon mindestens fünf Kopfschmerz-

anfälle aufgetreten, die der Beschreibung entsprechen?

Anzahl (n)

Prozent (\%)

- Ja 33

84,6

- Nein

3

- Keine Angabe

3

7,7

7,7

Gesamt

39

100,0

\section{Frage 31}

Wie lange leiden Sie an solchen

Kopfschmerzanfällen?

(Angabe in Jahren)

\begin{tabular}{cccc} 
Mittel & Minimum & Maximum & SD \\
\hline 12,31 & 1 & 40 & 11,987
\end{tabular}

Missings $=2$

\section{Frage 32}

An wie vielen Tagen pro Monat leiden

Sie durchschnittlich an entsprechenden

Kopfschmerzanfällen?

(Anzahl der Tage pro Monat)

Minimum Maximum

SD

$\begin{array}{llll}9,77 & 1 & 31 & 8,520\end{array}$

Missings $=1$ 


\section{Frage 33}

Treten bei Ihnen Kopfschmerzen auf, die

Antwort ,ja“

man wie folgt beschreiben kann?

Anzahl (n)

Prozent (\%)

a) Dauer ohne Behandlung: 30 min - 7 Tage

22

56,4

b) Beidseitiges Auftreten

41,0

c) Kann anfallsweise oder täglich auftreten

56,4

d) Drückender, ziehender, dumpfer Schmerz

22

46,2

e) Kein Erbrechen oder Übelkeit

43,6

Die Fragen 34 - 46 sollten nur von denjenigen Nutzern beantwortet werden, die auf die Fragen 33 a - e zumindest einmal mit "ja“ geantwortet hatten.

\section{Frage 34}

Dauern diese Kopfschmerzen gewöhnlich 30

Minuten bis 7 Tage an, wenn Sie kein

Medikament einnehmen oder eine Behandlung

erfolglos bleibt?

Anzahl (n)

Prozent (\%)

\begin{tabular}{clll}
\hline - Ja & 25 & 64,1 \\
- & Nein & 4 & 10,3 \\
- & Keine Angabe & 10 & 25,6 \\
\hline Gesamt & 39 & 100,0 \\
\hline
\end{tabular}

\section{Frage 35}

Können diese Kopfschmerzen einen dumpfen, drückenden bis ziehenden Charakter haben?

Anzahl (n) $\quad$ Prozent (\%)

\begin{tabular}{llll}
\hline - Ja & 18 & 46,2 \\
- & Nein & 9 & 23,1 \\
- & 12 & 30,8 \\
\hline Keine Angabe & 12 & 100,1 \\
\hline
\end{tabular}




\section{Frage 36}

Können Sie trotz dieser Kopfschmerzen Ihrer üblichen Tagesaktivität nachgehen?

Anzahl (n)

Prozent (\%)

\begin{tabular}{llll}
\hline - Ja & 16 & 41,0 \\
- & 13 & 33,3 \\
- Kein & 10 & 25,6 \\
\hline Gesame Angabe & 10 & 99,9 \\
\hline
\end{tabular}

\section{Frage 37}

Können diese Kopfschmerzen bei Ihnen beidseitig auftreten?

Anzahl (n) $\quad$ Prozent (\%)

\begin{tabular}{llll}
\hline - Ja & 23 & 59,0 \\
- & Nein & 6 & 15,4 \\
- & 10 & 25,6 \\
\hline \multirow{2}{*}{ Kesamt } & \multirow{2}{*}{39} & 100,0 \\
\hline
\end{tabular}

\section{Frage 38}

Bleiben diese Kopfschmerzen durch körperliche

Aktivität (z. B. Treppensteigen) unbeeinflusst?

Anzahl (n) $\quad$ Prozent (\%)

\begin{tabular}{llll}
\hline Ja & 10 & 25,6 \\
- & Nein & 19 & 48,7 \\
- & 10 & 25,6 \\
\hline \multirow{2}{*}{ Keine Angabe } & 10 & 99,9 \\
\hline
\end{tabular}

\section{Frage 39}

Können diese Kopfschmerzen von Übelkeit begleitet werden?

Anzahl (n)

Prozent (\%)

\begin{tabular}{llll}
\hline - Ja & 13 & 33,3 \\
- Nein & 16 & 41,0 \\
- Keine Angabe & 10 & 25,6 \\
\hline \multirow{2}{*}{ Gesamt } & 39 & 99,9
\end{tabular}




\section{Frage 40}

Können diese Kopfschmerzen von Erbrechen begleitet werden?

Anzahl (n)

Prozent (\%)

\begin{tabular}{llll}
\hline - Ja & 3 & 7,7 \\
- Nein & 26 & 66,7 \\
$-\quad$ Keine Angabe & 10 & 25,6 \\
\hline Gesamt & 39 & 100,0
\end{tabular}

\section{Frage 41}

Können diese Kopfschmerzen von Licht-

empfindlichkeit begleitet werden?

Anzahl (n) $\quad$ Prozent (\%)

\begin{tabular}{llll}
\hline - Ja & 14 & 35,9 \\
- & Nein & 14 & 35,9 \\
- & 11 & 28,2 \\
\hline Geine Angabe & 39 & 100,0 \\
\hline
\end{tabular}

\section{Frage 42}

\begin{tabular}{llc}
\hline $\begin{array}{l}\text { Können diese Kopfschmerzen von Lärm- } \\
\text { empfindlichkeit begleitet werden? }\end{array}$ & Anzahl (n) & Prozent (\%) \\
\hline - Ja & 19 & 48,7 \\
$-\quad$ Nein & 9 & 23,1 \\
$-\quad$ Keine Angabe & 11 & 28,2 \\
\hline Gesamt & 39 & 100,0 \\
\hline
\end{tabular}

\section{Frage 43}

Sind bei Ihnen schon mindestens zehn Kopfschmerzanfälle aufgetreten, die der angegebenen Beschreibung gleichen? Anzahl (n) $\quad$ Prozent (\%)

- Ja

- Nein

- Keine Angabe

24

39

61,5

12,8

25,6

Gesamt

99,9 


\section{Frage 44}

An wie vielen Tagen im Monat leiden

Sie durchschnittlich an solchen

Kopfschmerzanfällen?

(Anzahl der Tage pro Monat)

Mittel Minimum Maximum SD

$\begin{array}{llll}10,32 & 1 & 31 & 9,638\end{array}$

Missings $=11$

\section{Frage 45}

Leiden Sie schon länger an solchen

Kopfschmerzen?

Anzahl (n)

Prozent (\%)

\begin{tabular}{llll}
\hline - Ja & 25 & 64,1 \\
- & Nein & 2 & 5,1 \\
- & Keine Angabe & 12 & 30,8 \\
\hline Gesamt & 39 & 100,0 \\
\hline
\end{tabular}

Frage 46

Seit wie vielen Jahren leiden Sie an

solchen Kopfschmerzen?

(Angabe in Jahren)

Mittel Minimum Maximum SD

\begin{tabular}{lllll}
\hline 8,59 & 1 & 30 & 8,257 \\
\hline
\end{tabular}

Missings $=12$

\section{Frage 47}

\begin{tabular}{cccc}
\hline Ihr Geschlecht? & Anzahl (n) & Prozent (\%) \\
\hline - Weiblich & 34 & 87,2 \\
- Männlich & 4 & 10,3 \\
- Keine Angabe & 1 & 2,6 \\
\hline Gesamt & 39 & 100,1 \\
\hline
\end{tabular}




\section{Frage 48}

\begin{tabular}{rlcc}
\hline Welche Staatsangehörigkeit haben Sie? & Anzahl (n) & Prozent (\%) \\
\hline - & Deutsch & 33 & 84,6 \\
- & Spanisch & 1 & 2,6 \\
- & Schweizerisch & 1 & 2,6 \\
- Österreichisch & 1 & 2,6 \\
- & Kroatisch & 1 & 2,6 \\
- & Keine Angabe & 2 & 5,1 \\
\hline \multirow{2}{*}{ Gesamt } & & 39 & 100,1 \\
\hline
\end{tabular}

\section{Frage 49}

\begin{tabular}{|c|c|c|c|c|c|}
\hline \multirow{2}{*}{\multicolumn{2}{|c|}{ Wie alt sind Sie? }} & Mittel & Minimum & Maximum & SD \\
\hline & & 33,76 & 17 & 57 & 9,887 \\
\hline \multicolumn{6}{|c|}{ Missings $=1$} \\
\hline \multicolumn{6}{|c|}{ Frage 50} \\
\hline Leb & en Sie mit einem & «sammen? & Anzahl (n) & \multicolumn{2}{|c|}{ Prozent (\%) } \\
\hline & $\mathrm{Ja}$ & & 25 & \multicolumn{2}{|c|}{64,1} \\
\hline & Nein & & 13 & \multicolumn{2}{|c|}{33,3} \\
\hline- & Keine Angabe & & 1 & \multicolumn{2}{|c|}{2,6} \\
\hline Ges & amt & & 39 & \multicolumn{2}{|c|}{100,0} \\
\hline
\end{tabular}

\section{Frage 51}

Wie viele Personen leben ständig in Ihrem Haushalt, Sie selbst eingeschlossen?

\begin{tabular}{cccc} 
Mittel & Minimum & Maximum & SD \\
\hline 2,59 & 1 & 7 & 1,443
\end{tabular}

Missings $=2$ 


\subsection{Grundauszählung der Anfragenanalyse}

Stichprobenpopulation (Anfragen)

\begin{tabular}{|c|c|c|c|c|}
\hline & & Anzahl (n) & \multicolumn{2}{|c|}{ Prozent (\%) } \\
\hline \multicolumn{5}{|l|}{ Geschlecht } \\
\hline - Weiblich & & 548 & & \\
\hline - Männlich & & 95 & & \\
\hline - Keine Angabe & & 192 & & \\
\hline \multicolumn{5}{|l|}{ Namensangabe } \\
\hline - Voller Name/ Nachname & & 107 & & \\
\hline - Vorname & & 495 & & \\
\hline - Keine Angabe & & 233 & & \\
\hline \multicolumn{5}{|l|}{ Angabe der E-Mail-Adresse } \\
\hline \multirow{3}{*}{$\begin{array}{ll}- & \text { Ja } \\
- & \text { Nein }\end{array}$} & & 61 & \multirow{2}{*}{\multicolumn{2}{|c|}{$\begin{array}{l}7,3 \\
92,7\end{array}$}} \\
\hline & & 774 & & \\
\hline & Mittel & Minimum & Maximum & SD \\
\hline Alter der Anfragensteller (Jahre) & 33,23 & 14 & 68 & 11,288 \\
\hline (Diese freiwillige Angabe wurde & 88 Pers & $(22,5 \%) g$ & cht.) & \\
\hline
\end{tabular}

\section{Charakteristika der Anfragen}

Anzahl (n) Prozent (\%)

Bezug der Anfragen

- Auf Anfragesteller selbst

688

82,4

- Auf Familie, Freunde, Bekannte

90

10,8

- Auf Anfragesteller und andere Personen

- Auf keine personen-gebundenen Aspekte

- Keine eindeutige Zuordnung

1

53

0,1

3

6,3

$3 \quad 0,4$

Antwort auf die Anfrage

- Durch den Experten

- Durch andere Nutzer

- Erneute Reaktion des Anfragestellers

87

10,4

Bezugnahme der Anfragen auf frühere Fragen

- Ja

42

5,0

- Nein

793

95,0

\begin{tabular}{lcccc}
\hline & Mittel & Minimum & Maximum & SD \\
\hline Anzahl der Wörter & 107,91 & 5 & 1075 & 88,410 \\
\hline
\end{tabular}


Anzahl (n)

Prozent (\%)

Symptombeschreibung

Bestimmte Medikamente/ Therapien

Mögliche Therapien

Suche nach psych. Beistand/ Hilfe

Ursachen der KS

128

15,3

Sonstiges

Diagnostik/ U-Ergebnisse

(Fach-) Arzt-/ Therapieempfehlung

Hormonelle Ursachen/ Therapie

68

63

8,1

Kopfschmerzen bei Kindern

58

Trigger/ Auslöser/ Verstärker

45

Vorbeugung/ Prophylaxe

44

Alternative Therapien

34

Zusammenhang mit bestimmten Erkrankungen

34

Schwangerschaft und Kopfschmerz

18

Einfluss von Lebensgewohnheiten

6,9

5,4

5,3

4,1

4,1

2,2

2,0 


\section{Literaturverzeichnis}

Abbate-Daga G, Fassino S, Lo Giudice R, Rainero I, Gramaglia C, Marech L, Amianto F, Gentile S, Pinessi L (2007): Anger, Depression and personality dimensions in patients with migraine without aura. Psychother Psychosom 76 (2), 112 - 128

Akerkar SM, Bichile LS (2004): Health information on the Internet: Patient empowerment or patient deceit? Indian J Med Sci $\underline{58}, 321$ - 326

Anderson R, Barbara A, Feldman S (2007): What patients want: A content analysis of key qualities that influence patient satisfaction. J Med Pract Manage 22 (5), 255 - 261

Antilla P (2006): Tension-type headache in childhood and adolescence. Lancet Neurol $\underline{5}$ (3), $268-274$

Arican O (2007): E-dermatology: Emails about dermatological diseases in the Internet. J Dermatol $\underline{34}, 375-380$

Baskin SM (2007): Managing the "difficult" headache patient. Neurol Sci $\underline{28}$, S78 - S83

Bauer KA (2002): Using the Internet to empower patients and to develop partnerships with clinicians. World Hosp Health Serv $\underline{38(2)}, 2$ - 10

Bennett NL, Casebeer LL, Zheng S, Kristofco R (2006): Information-seeking behaviours and reflective practice. J Contin Educ Health Prof 26(2), 120 - 127

Besell TL, McDonald S, Silagy C, Anderson J, Hiller J, Sansom L (2002): Do Internet interventions for consumers cause more harm than good? A systematic review. Health Expect $\underline{5}, 28-37$

Bianco A, Parente MM, De Caro E, lannacchero R, Cannistra U, Angelillo IF (2005): Evidence-based medicine and headache patient management by general practitioners in Italy. Cephalalgia 25 (10), $767-775$

Bigal ME, Serrano D, Reed M, Lipton RB (2008): Chronic migraine in the population: burden, diagnosis, and satisfaction with treatment. Neurology $\underline{71}$ (8), 559 - 566 
Bloom BS, Iannacone RC (2006): Changing availability and cost of Internet physician consultations and prescription medications. Med Inform Internet Med 31(4), 247 - 253

Blyth FM, March LM, Brnabic AJ, Cousins MJ (2004): Chronic pain and frequent use of health care. Pain $\underline{111(1-2)}, 51-58$

Bodenheimer T, Grumbach K (2003): Electronic technology: a spark to revitalize primary care? JAMA $290(2), 259-264$

Breslau N, Rasmussen BK (2001): The impact of migraine: Epidemiology, risk factors, and

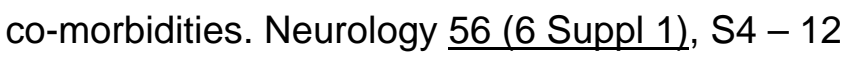

Carney PA, Poor DA, Schifferdecker KE, Gephart DS, Brooks WB, Nierenberg DW (2004): Computer use among community-based primary care physicians preceptors. Acad Med $\underline{79(6)}, 580-590$

Cottrell CK, Drew JB, Waller SE, Holroyd KA, Brose JA, O’Donnell FJ (2002): Perceptions and needs of patients with migraine. J Fam Pract $\underline{51}, 142-147$

Darer JD, Hwang W, Pham HH, Bass EB, Anderson G (2004): More training needed in chronic care: a survey of US physicians. Acad Med $\underline{79}$ (6), 541 - 548

Davies GM, Santanello N, Lipton R (2000): Determinants of patient satisfaction with migraine therapy. Cephalalgia 20 (6), $554-560$

Delbanco T, Sands DZ (2004): Electrons in flight - E-Mail between doctors and patients. N Engl J Med 350; 17, $1705-1707$

Delic D, Polasek O, Kern J (2006): Internet health-information seekers in Croatia - who, what, and why? Med Inform Internet Med 31(4), 267 - 273

Devineni T, Blanchard EB (2005): A randomized controlled trial of an Internet-based treatment for chronic headache. Behav Res Ther 43 (3), 277 - 292

Dueland AN, Leira R, Cabelli ST (2005): The impact of migraine on psychological wellbeing of young women and their communication with physicians about migraine: a multinational study. Curr Med Res Opin 21 (8), 1297 - 1305 
Elder N, Ricer, R, Tobias B (2006): How respected family physicians manage difficult patient encounters. J Am Board Fam Med 19 (6), 533 - 541

Eysenbach G, Diepgen TL (1999): Patients looking for information on the Internet and seeking teleadvice: motivation, expectations, and misconceptions as expressed in e-mails sent to physicians. Arch Dermatol $\underline{135(2)} 151-156$

Farmer A, Gibson O, Hayton P, Bryden K, Dudley C, Neil A, Tarassenko L (2005): A realtime, mobile phone-based telemedicine system to support young adults with type 1 diabetes. Inform Prim Care 13 (3), $171-177$

Frediani F, Villani V (2007): Migraine and depression. Neurol Sci 28 Suppl 2, S161 - 165

Freed DH (2003): Patient-physician e-mail: passion or fashion? Health Care Manag (Frederick) $\underline{22(3)}, 265-274$

Freitag FG (2007): The cycle of migraine: patients' quality of life during and between migraine attacks. Clin Ther 29 (5), $939-949$

Genzen, JR (1998): The Internet and migraine: headache resources for patients and physicians. Headache $\underline{38}, 312-314$

Gibbs TS, Fleischer AB Jr, Feldman SR, Sam MC, O'Donovan CA (2003): Health care utilization in patients with migraine: demographics and patterns of care in the ambulatory setting. Headache $\underline{43(4)}, 330-335$

Giffin N (2002): Surfing for headache. J Neurol Neurosurg Psychiatry $\underline{72}$, ii9 - ii9

Göbel H (1994): Paper-pencil tests for retrospective and prospective evaluation of primary headache on the basis of the IHS criteria. Headache $\underline{34}, 564-568$

Göbel H, Petersen-Braun M, Soyka D (1994): The epidemiology of headache in Germany: a nationwide survey of a representative sample on the basis of the headache classification of the International Headache Society. Cephalalgia 14, 97 - 106

Göbel H, Gessner U, Petersen-Braun M, Weingärtner U (2007): Acetylsalicylsäure bei der Selbstmedikation von Migränekopfschmerzen: Eine apothekenbasierte Analyse. Schmerz 21, $49-56$ 
Goldberg LD (2005): The cost of migraine and its treatment. Am J Manag Care 11, S62 S67

Google (2008): Unternehmensprofil. http://www.google.de/intl/de/corporate/, zugegriffen am 28.10.2008

Gottlieb S (2004): US doctors want to be paid for email communication with patients. BMJ $\underline{328}, 1155$

Grazi GL (2006): Web relationships between physicians and individuals seeking information on hepatopancreatobiliary diseases. Arch Surg 141 (12), 1176 - 1182

Gupta MX, Silberstein SD, Young WB, Hopkins M, Lopez BL, Samsa Group (2007): Less is not more: underutilization of headache medications in a university hospital emergency department. Headache 47 (8), $1125-1133$

Hasse LA, Ritchey PN, Smith R (2002): Predicting the number of headache visits by type of patient seen in family practice. Headache $\underline{42(8)}, 738-746$

Hawkins K, Wang S, Rupnow M (2008): Direct cost burden among insured US employees with migraine. Headache $\underline{48(4)}, 553-563$

Headache Classification Subcommittee of the International Headache Society (2004): The International Classification of Headache Disorders, $2^{\text {nd }}$ Edition. Cephalalgia 24 suppl 1,1 160

Houston TK, Sands DZ, Nash BR, Ford DE (2003): Experiences of physicians who frequently use e-mail with patients. Health Commun $\underline{15}$ (4), $515-525$

Kobak KA, Ketzelnick DJ, Sands G, King M, Greist JJ, Dominski M (2005): Prevalence and burden of illness of migraine in managed care patients. J Manag Care Pharm 11 (2), $124-136$

Kommission „Leitlinien der Deutschen Gesellschaft für Neurologie“: Therapie der Migräne; in: Leitlinien für die Diagnostik und Therapie in der Neurologie; 4. überarbeitete Auflage, Georg Thieme Verlag Stuttgart 2008 a, 579 - 596 
Kommission „Leitlinien der Deutschen Gesellschaft für Neurologie“: Diagnostik und apparative Zusatzuntersuchungen bei Kopfschmerzen; in: Leitlinien für die Diagnostik und Therapie in der Neurologie; 4. überarbeitete Auflage, Georg Thieme Verlag Stuttgart 2008 b, $556-562$

Kongsved SM, Basnov M, Holm-Christensen K, Hjollund NH (2007): Response rate and completeness of questionnaires: a randomized study of Internet versus paper-and-pencil versions. J Med Internet Res 9 (3), e25

Krippendorff K: Content analysis: an introduction to its methodology, 1. Auflage; Sage Publications, Beverly Hills, California, USA 1980

Lake AE (1999): Psychological impact: the personal burden of migraine. Am J Manag Care 5 (2 Suppl), S111 - 121

Lanteri-Minet M (2005): What do patients want from their acute migraine therapy? Eur

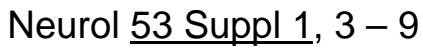

Larner AJ (2006): Searching the Internet for medical information: frequency over time and by age and gender in an outpatient population in the UK. J Telemed Telecare 12(4), 186 188

Latinovic R, Gulliford M, Ridsdale L (2006): Headache and migraine in primary care: consultation, prescription, and referral rates in a large population. J Neurol Neurosurg Psychiatry $\underline{77(3)}, 385-387$

Leece P, Bhandari M, Sprague S, Swiontkowski MF, Schemtisch EH, Tornetta P, Devereaux PJ, Guyatt GH (2004): Internet versus mailed questionnaires: a controlled comparison (2). J Med Internet Res $\underline{6(4)}$, e39

Leiper DA, Elliott AM, Hannaford PC (2006): Experiences and perceptions of people with headache: a qualitative study. BMC Fam Pract $\underline{7}, 27$

Lenert AL, Looman T, Agoncillo T, Nguyen M, Sturley A, Jackson CM (2002): Potential validity of conducting research on headache in Internet populations. Headache $\underline{42}, 200-$ 203 
Leonardi M, Steiner TJ, Scher AT, Lipton RB (2005): The global burden of migraine: measuring disability in headache disorders with WHO's Classification of Functioning, Disability and Health (ICF). J Headache Pain $\underline{6(6)}, 429$ - 440

Linssen C, Schook RM, The AM, Lammers E, Festen J, Postmus PE (2007): A web site on lung cancer: who are the users and what are they looking for? J Thorac Oncol 2 (9), $813-818$

Lipton RB, Stewart WF (1999): Acute migraine therapy: Do doctors understand what patients with migraine want from therapy? Headache 39 (Suppl 2), S20 - S26

Lipton RB, Amatniek JC, Ferrari MD, Gross M (1994): Migraine. Identifying and removing barriers to care. Neurology 44 (6 Suppl 4), S63 - 68

Lipton RB, Stewart WF, Simon D (1998): Medical consultation for migraine: results from the American Migraine Study. Headache 38 (2), 87 - 96

Lipton RB, Stewart WF, Diamond S, Diamond ML, Reed M (2001): Prevalence and burden of migraine in the United States: data from the American Migraine Study II. Headache $\underline{41(7)}, 646-657$

Lipton RB, Scher Al, Kolodner K, Liberman J, Steiner TJ, Stewart WF (2002): Migraine in the United States: epidemiology and patterns of health care use. Neurology $\underline{58(6)}, 885-$ 894

Lipton RB, Silberstein SD, Saper JR, Bigal ME, Goadsby PJ (2003 a): Why headache treatment fails. Neurology $\underline{60(7)}, 1064-1070$

Lipton RB, Scher Al, Steiner TJ, Bigal ME, Kolodner K, Liberman JN, Stewart WF (2003 b): Patterns of health care utilization for migraine in England and in the United States. Neurology $\underline{60(3)}, 441-448$

Lipton RB, Bigal ME, Diamond M, Freitag F, Reed ML, Stewart WF, AMPP Advisory Group (2007): Migraine prevalence, disease burden, and the need for preventive therapy. Neurology $\underline{68(5)}, 343-349$

Lyngberg AC, Rasmussen BK, Jorgensen T, Jensen R (2005 a): Incidence of primary headache: a Danish epidemiologic follow-up study. Am J Epidemiol 161 (11), 1066 - 1073 
Lyngberg AC, Rasmussen BK, Jorgensen T, Jensen R (2005 b): Has the prevalence of migraine and tension-type headache changes over a 12-year period? A Danish population survey. Eur J Epidemiol 20 (3), 243 - 249

MacGregor EA (1997): The doctor and the migraine patient: improving compliance. Neurology 48 (3 Suppl 3), S16 - 20

Malone M, Harris R, Hooker R, Tucker T, Tanna N, Honnor S (2004): Health and the Internet - changing boundaries in primary care. Fam Pract 21 (2), 189 - 191

Manzoni GC, Torelli P (2007): The patient-physician relationship in the approach to therapeutic management. Neurol Sci 28 Suppl 2, S130 - 133

Martins KM, Bordini CA, Bigal ME, Speciali JG (2006): Migraine in the elderly: a comparison with migraine in young adults. Headache $\underline{46(2)}, 312-316$

Masters K (2008): For what purpose and reasons do doctors use the Internet: a systematic review. Int J Med Inform $\underline{77 \text { (1) }}, 4$ - 16

Mavis BE, Brocato JJ (1998): Postal surveys versus electronic mail surveys. The tortoise and the hare revisited. Eval Health Prof 21 (3), 395 - 408

Mildenstein K (2009): DEGAM-Leitlinie Kopfschmerzen, unautorisierter Entwurf vor der Publikation. Deutsche Gesellschaft für Allgemein- und Familienmedizin (DEGAM).

Molarius A, Tegelberg $\AA$ (2006): Recurrent headache and migraine as a public health problem - a population-based study in Sweden. Headache $\underline{46}, 73-81$

Mueller LL (2007): Diagnosing and managing migraine headache. J Am Osteopath Assoc 107 (10 Suppl 6), ES10 - 16

Murray E, Lo B, Pollack L, Donelan K, Catania J, Lee K, Zapert K, Turner R (2003): The impact of health information on the Internet on health care and the physician-patientrelationship: National U.S. survey among 1.050 U.S. physicians. J Med Internet Res $\underline{5 \text { (3), }}$ e17

Neu IS (2001): Differential diagnosis and therapy of headache in general practice. Anamnesis is the alpha and omega. MMW Fortschr Med 143 Suppl 2, $36-40$ 
Netcraft (2008): Web Server Survey. www.netcraft.com, zugegriffen am 28.10.2008

Neville RG, Marsden W, McCowan C, Pagliari C, Mullen H, Fannin A (2004 a): A survey of GP attitudes to and experiences of email consultations. Inform Prim Care 12(4), 201 206

Neville RG, Marsden W, McCowan C, Pagliari C, Mullen H, Fannin A (2004 b): Email consultations in general practice. Inform Prim Care 12 (4), 207 - 214

Patt, MR, Houston TK, Jenckes MW, Sands DZ, Ford DE (2003): Doctors who are using e-mail with their patients: a qualitative exploration. J Med Internet Res $\underline{5(2)}$, e9

Pemberton PJ, Goldblatt J (1998): The Internet and the changing role of doctors, patients and their websurfing skills. Med J Aust $\underline{169}, 594-595$

Pennekamp PH, Diedrich O, Schmitt O, Kraft CN (2006): Prävalenz und Stellenwert der

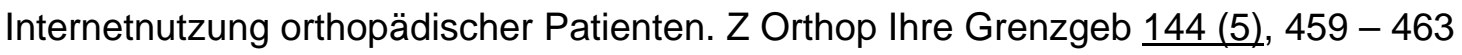

Peroutka SJ (2001): Analysis of Internet sites for headache. Cephalalgia 21, 20 - 24

Peterlin BL, Gamini-Suarez E, Levin M (2007): Cluster headache: a review of online resources for patients and clinicians. Curr Pain Headache Rep 11(2), 148 - 153

Peterlin BL, Gambini-Suarez E, Lidicker J, Levin M (2008): An analysis of cluster headache information provided on Internet websites. Headache 48 (3), 378 - 384

Pfaffenrath V, Fendrick K, Vennemann M, Meinsiger C, Ladwig KH, Evers S, Straube A, Hoffmann W, Berger K (2008): Regional variations in the prevalence of migraine and tension-type headache applying the new IHS criteria: the German DMKG Headache Study. Cephalalgia (im Druck, vorab im Internet publiziert)

Pradalier A, Auray JP, El Hasnaoui A, Alzahouri K, Dartigues JF, Duru G, Henry P, Lanteri-Minet M, Lucas C, Chazot G, Gaudin AF (2004): Economic impact of migraine and other episodic headaches in France: data from the GRIM2000 study. Pharmacoeconomics $\underline{22(15)}, 985-999$

Radtke A, Neuhauser H (2009): Prevalence and burden of headache and migraine in Germany. Headache 49 (1), 79 - 89 
Ramos Martinez A, Asensio Vegas A, de la Serna Fernandez de Cordoba JL (2004): Internet physician's offices: main reasons for consultations and differences with primary care offices. Rev Clin Esp 204 (4), 198 - 201

Rasmussen BK (1999): Epidemiology and socio-economic impact of headache. Cephalalgia 19 Suppl $25,20-23$

Rasmussen BK, Olesen J (1992): Migraine with aura and migraine without aura: an epidemiological study. Cephalalgia $\underline{12}$ (4), $221-228$

Rasmussen BK, Jensen R, Schroll M, Olesen J (1991): Epidemiology of headache in a general population - a prevalence study. J Clin Epidemiol 44 (11), 1147 - 1157

Recupero PR, Rainey SE (2006): Characteristics of e-therapy web sites. J Clin Psychiatry $\underline{67(9)}, 1435-1440$

Ridsdale L, Doherty J, McCrone P, Seed P, Headache Innovation and Evaluation Group (2008): A new GP with special interest headache service: observational study. Br J Gen

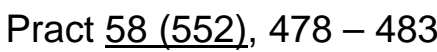

Rozen TD (2006): Migraine prevention: what patients want from medication and their physicians (a headache specialty clinic perspective). Headache 45 (5), $750-753$

Sadovsky R, Dodick DW (2005): Identifying migraine in primary care settings. Am J Med 118 Suppl 1, 11 S - 17 S

Shuyler KS, Knight KM (2003): What are patients seeking when they turn to the Internet? Qualitative content analysis of questions asked by visitors to an orthopaedics web site. $\mathrm{J}$ Med Internet Res 5 (4), e24

Sim NZ, Kitteringham L, Spitz L, Pierro A, Kiely E, Drake D, Curry J (2007): Informations on the World Wide Web - how useful is it for parents? J Pediatr Surg 42 (2), $305-312$

Sittig DF, King S, Hazlehurst BL (2001): A survey of a patient-provider e-mail communication: what do patients think? Int J Med Inform $\underline{61}$ (1), 71 - 80

Smith PK, Fox AT, Davies P, Hamidi-Manesh L (2006): Cyberchondriacs. Int J Adolesc Med Health $\underline{18(2)}, 209-213$ 
Stark RJ, Valenti L, Miller GC (2007): Management of migraine in Australian general practice. Med J Aust 187 (3), 142 - 146

Statistisches Bundesamt Deutschland (2008 a): Geräte, Anschlüsse und Internetzugang in Privathaushalten: Deutschland, Früheres Bundesgebiet/ Neue Länder, Jahre. GENESIS-Online Datenbank, Code 63931-0001, zugegriffen am 28.10.2008

Statistisches Bundesamt Deutschland (2008 b): Personen, die das Internet im 1. Quartal genutzt haben: Deutschland, Früheres Bundesgebiet/ Neue Länder, Jahre, Nutzung des Internet. GENESIS-Online Datenbank, Code 63931-0007, zugegriffen am 28.10.2008

Steiner TJ, Scher Al, Stewart WF, Kolodner K, Liberman J, Lipton RB (2003): The prevalence and disability burden of adult migraine in England and their relationship to age, gender and ethnicity. Cephalalgia $\underline{23}(7), 519-527$

Stevenson FA, Kerr C, Murray E, Nazareth I (2007): Information from the Internet and the doctor-patient relationship: the patient perspective - a qualitative study. BMC Fam Pract $\underline{8}, 47$

Stewart WF, Shechter A, Rasmussen BK (1994): Migraine prevalence. A review of population-based studies. Neurology 44 (6 Suppl 4), S17 - 23

Stovner LJ, Hagen K, Jensen R, Katsarava Z, Lipton R, Scher A, Steiner T, Zwart JA (2007): The global burden of headache: a documentation of headache prevalence and disability worldwide. Cephalalgia 27(3), $193-210$

Strom L, Pettersson R, Andersson G (2000): A controlled trial of self-help treatment of recurrent headache conducted via the Internet. J Consult Clin Psychol 68 (4), 722 - 727

Thomas E, Boardman HF, Ogden H, Millson DS, Croft PR (2004): Advice and care for headaches: Who seeks it, who gives it? Cephalalgia 24, $740-752$

Truell A, Bartlett J, Alexander M (2002): Response rate, speed, and completeness: A comparison of Internet-based and mail surveys. Behav Res Methods Instrum Comput $\underline{34}$ (1), $46-49$

Umefjord G, Petersson G, Hamberg K (2003): Reasons for consulting a doctor on the Internet: web survey of an Ask the Doctor Service. J Med Internet Res 5 (4), e26 
Umefjord G, Malker H, Olofsson N Hensjo LO, Petersson G (2004): Primary care physicians' experiences of carrying out consultations on the Internet. Inform Prim Care 12 (2), $85-90$

Umefjord G, Hamberg K, Malker H, Petersson G (2006): The use of an Internet-based Ask the Doctor Service involving family physicians: evaluation by a web survey. Fam Pract $\underline{23}$, $159-166$

Umefjord G, Sandstrom H, Malker H, Petersson G (2008): Medical text-based consultations on the Internet: A 4-year study. Int J Med Inform 77 (2), 114 - 121

Underhill C, Mckeown L (2008): Getting a second opinion: health information and the Internet. Health Rep 19 (1), 65 - 69

Van Den Kerkhof EG, Parlow JL, Goldstein DH, Milne B (2004): In Canada, anesthesiologists are less likely to respond to an electronic, compared to a paper questionnaire. Can J Anaesth $\underline{51(5)}, 449-454$

Van Woerkum CMJ (2003): The Internet and primary care physicians: coping with different expectations. Am J Clin Nutr 77 (suppl), $1016 \mathrm{~S}-1018 \mathrm{~S}$

Vinding G, Zeeberg P, Lyngberg A, Nielsen R, Jensen R (2007): The burden of headache in a patient population from a specialized headache centre. Cephalalgia $\underline{27}(3), 263-270$

Vinker S, Weinfass M, Kasinetz LM, Kitai E, Kaiserman I (2007): Web-based questionanswering service of a family physician - the characteristics of queries in a noncommercial open forum. Med Inform Internet Med 32(2), 123 - 129

Von Korff M, Lin EH, Fenton JJ, Saunders K (2007): Frequency and priority of pain patient's health care use. Clin J Pain 23 (5), $400-408$

Walling AD, Woolley DC, Molgaard C, Kallail KJ (2005): Patient satisfaction with migraine management by family physicians. J Am Board Fam Pract $\underline{18}, 563-566$

Weiss N (2004): E-mail consultation: clinical, financial, legal, and ethical implications. Surg Neurol $\underline{61(5)}, 455-459$ 
White CB, Moyer CA, Stern DT, Katz SJ (2004): A content analysis of e-mail communication between patients and their providers: patients get the message. J Am Med Inform Assoc 11(4), $268-269$

Wiendels NJ, Knuistingh Neven A, Rosendaal FR, Spinhoven P, Zitman FG, Assendelft WJ, Ferrari MD (2006 a): Chronic frequent headache in the general population: prevalence and associated factors. Cephalalgia 26 (12), $1434-1442$

Wiendels NJ, van Haestregt A, Neven AK, Spinhoven P, Zitman F, Assendelft W, Ferrari M (2006 b): Chronic frequent headache in the general population: comorbidity and quality of life. Cephalalgia $\underline{23}$ (12), $1443-1450$

Wilm S, Brockmann S, Spannaus-Sakic C, Altiner A, Hemming B, Abholz HH (2008): Machen Hausärzte Unterschiede, wenn sie mit Kopfschmerzpatienten umgehen? Eine Querschnittsstudie mit ängstlich oder neutral gespielten standardisierten Patienten. Z Allg Med $\underline{84}, 273-279$

Wyatt JC (2000): When to use web-based surveys. J Am Med Inform Assoc 7 (4), 426 429 


\section{Danksagung}

Ich danke meinem Doktorvater, Herrn Prof. Dr. med. Martin Scherer, für die Betreuung der Dissertation, seine wertvollen Vorschläge und die konstruktive Kritik.

Bei Herrn Prof. Dr. disc. pol. Wolfgang Himmel möchte ich mich für seine freundliche Hilfe und Unterstützung während den verschiedenen Phasen der Dissertation bedanken.

Außerdem danke ich der Redaktion der Lifeline-Homepage der Business Solutions Medicine Online (BSMO), vertreten durch Frau Tarja Wündrich, für ihre Unterstützung bei der Durchführung der Internetbefragung.

Herrn Dr. med. Klas Mildenstein danke ich für seine Anregungen zu der vorliegenden Arbeit und sein Einverständnis, seine Arbeit im Expertenrat derart zu untersuchen.

Ferner danke ich Frau Irene Kühne aus der Abteilung Allgemeinmedizin der Universität Göttingen für die technische Umsetzung des Online-Fragebogens und ihre Hilfe während der Erhebungsphase. 


\section{Lebenslauf}

Am 01. August 1980 wurde ich, Sophie Zitterbart, als erstes von drei gemeinsamen Kindern von Prof. Gerrit Zitterbart, Pianist, und Ilsemarie Zitterbart, geb. Habermann, Gesprächstherapeutin, in Göttingen geboren.

Nach dem Besuch der Grundschule Höltyschule Göttingen und der Orientierungsstufe Lutherschule Göttingen besuchte ich das Theodor-Heuss-Gymnasium in Göttingen, welches ich 1999 mit der Allgemeinen Hochschulreife abschloss.

Im Wintersemester 1999/ 2000 nahm ich mein Studium der Humanmedizin an der Universität Göttingen auf und bestand im Wintersemester 2002/ 2003 die Ärztliche Vorprüfung. Während der klinischen Ausbildung absolvierte ich mehrere Famulaturen, darunter auch eine Auslandsfamulatur in der Neurologie und der Pädiatrie im South Bend Memorial Hospital, South Bend, Indiana, USA. Im Frühjahr 2004 bestand ich die 1. Ärztliche Prüfung nach alter Approbationsordnung. Während des Praktischen Jahres absolvierte ich die Fächer Gynäkologie und Geburtshilfe, Chirurgie und Innere Medizin im AlbertSchweitzer-Krankenhaus Northeim mit einem Auslandsaufenthalt während des Tertials der Inneren Medizin an der Galway University Clinic, Galway, Irland.

Im Frühjahr 2007 beendete ich mein Medizinstudium mit der 2. Ärztlichen Prüfung nach neuer Approbationsordnung. Seit Oktober 2007 arbeite ich nun als Assistenzärztin in der Frauenklinik der Ammerlandklinik Westerstede und absolviere dort meine Facharztausbildung in der Gynäkologie und Geburtshilfe. 